\title{
Space Shift Keying Modulation for MIMO Channels
}

\author{
Jeyadeepan Jeganathan \\ A Thesis \\ in \\ The Department \\ of \\ Electrical and Computer Engineering \\ Presented in Partial Fulfillment of the Requirements \\ for the Degree of Master of Applied Science \\ Concordia University \\ Montreal, Quebec, Canada
}

August 2008

(C) Jeyadeepan Jeganathan, 2008 


$\begin{array}{ll}\begin{array}{l}\text { Library and } \\ \text { Archives Canada }\end{array} & \begin{array}{l}\text { Bibliothèque et } \\ \text { Archives Canada }\end{array} \\ \begin{array}{l}\text { Published Heritage } \\ \text { Branch }\end{array} & \begin{array}{l}\text { Direction du } \\ \text { Patrimoine de l'édition }\end{array} \\ \begin{array}{l}\text { 395 Wellington Street } \\ \text { Ottawa ON K1A 0N4 } \\ \text { Canada }\end{array} & \begin{array}{l}\text { 395, rue Wellington } \\ \text { Ottawa ON K1A 0N4 } \\ \text { Canada }\end{array}\end{array}$

Your file Votre référence ISBN: 978-0-494-45706-1 Ourfile Notre référence ISBN: 978-0-494-45706-1

NOTICE:

The author has granted a nonexclusive license allowing Library and Archives Canada to reproduce, publish, archive, preserve, conserve, communicate to the public by telecommunication or on the Internet, loan, distribute and sell theses worldwide, for commercial or noncommercial purposes, in microform, paper, electronic and/or any other formats.

The author retains copyright ownership and moral rights in this thesis. Neither the thesis nor substantial extracts from it may be printed or otherwise reproduced without the author's permission.
AVIS:

L'auteur a accordé une licence non exclusive permettant à la Bibliothèque et Archives Canada de reproduire, publier, archiver, sauvegarder, conserver, transmettre au public par télécommunication ou par l'Internet, prêter, distribuer et vendre des thèses partout dans le monde, à des fins commerciales ou autres, sur support microforme, papier, électronique et/ou autres formats.

L'auteur conserve la propriété du droit d'auteur et des droits moraux qui protège cette thèse. $\mathrm{Ni}$ la thèse ni des extraits substantiels de celle-ci ne doivent être imprimés ou autrement reproduits sans son autorisation.
In compliance with the Canadian Privacy Act some supporting forms may have been removed from this thesis.

While these forms may be included in the document page count, their removal does not represent any loss of content from the thesis.
Conformément à la loi canadienne sur la protection de la vie privée, quelques formulaires secondaires ont été enlevés de cette thèse.

Bien que ces formulaires aient inclus dans la pagination, il n'y aura aucun contenu manquant.

\section{Canada}




\title{
Abstract \\ Space Shift Keying Modulation for MIMO Channels
}

\author{
Jeyadeepan Jeganathan
}

In this thesis, we analyze modulation techniques that exploit multiple antennas in wireless communication. We first, study the so-called spatial modulation (SM) technique for MIMO channels. Since the original SM detector is based on an ad hoc design, and only functions under some artificial assumptions about the channel, we derive the optimal detector for SM. The new detector performs significantly better than the original ( $\sim 4 \mathrm{~dB}$ gain), and we support our results by deriving a closed form expression for the average bit error probability. As well, we show that SM with the optimal detector achieves better performance gains $(\sim 1.5-3 \mathrm{~dB})$ over popular multiple antenna systems.

We then introduce space shift keying (SSK), a new modulation scheme based on the SM concept. SSK exploits fading in multiple input multiple output (MIMO) channels to provide better performance over conventional amplitude/phase modulation (APM) techniques. In SSK, only the antenna indices, and not the symbols themselves, relay information. This absence of symbol information eliminates the transceiver elements necessary for APM transmission and detection (such as coherent 
detectors). As well, the simplicity involved in modulation reduces detection complexity compared to that of SM, while achieving almost identical performance gains.

Throughout the thesis, we illustrate SSK's strength by studying its interaction with the fading channel, and obtain tight upper bounds on bit error probability. To improve performance, adaptive forms of SSK are also presented, including a symbol design technique, and an antenna selection scheme. We also illustrate SSK's performance under channel estimation error, and spatial correlation. Analytical and simulation results show performance gains over APM systems ( $3 \mathrm{~dB}$ at a bit error rate of $10^{-5}$ ), making SSK an interesting candidate for wireless applications.

We then present SSK coded modulation (SSK-CM) to integrate coding for practical wireless systems. In particular, we present a bit interleaved CM (BICM) system using iterative decoding. We illustrate SSK-CM capacity improvements over APM, and derive upper bounds on SSK-CM's performance. We also analytically present SSK's coded diversity advantage over APM, where significant performance gains are observed (up to $9 \mathrm{~dB}$ ), motivating SSK-CM's integration in future wireless standards. 
Dedicated to Navinta, the love of my life, who has always supported me... 


\section{Acknowledgments}

First and foremost, I would like to extend my greatest appreciation and thanks to my primary supervisor, Dr. Ali Ghrayeb, for his guidance and support. I have come to know him as a kind, and understanding individual, who took genuine interest in my thesis work. I am certain that, without his supervision, I would not have accomplished nearly as much as I did. Secondly, I would also like to extend my full appreciation and thanks to my secondary supervisor, Dr. Leszek Szczecinski, for all his time and effort. He has been an exceptional advisor, who truly tried to help me with all his knowledge. He has contributed so much to my understanding, and there is no doubt, that, without his significant assistance, this thesis would not be where it is today.

I would like to thank the committee members, Dr. J. Zhang, Dr. R. Soleymani, and Dr. A. Ben Hamza, for their review of this thesis.

I would like to thank Xiangnian Zeng for her kind support as well. She has guided me with her experience, and has always been a friendly face to see in the lab. I would also like to thank May Gomaa, Pooyan Haghighat, Mohamed El-fituri, Ghaleb Al Habianand, and Mohamed Abuthinien from our research group for all of their input and support. 
Finally, I would like to thank my parents for their love, and encouragement, and my sister Janita, for keeping me entertained in times of need. Of course, I am also indebted to Navinta Dua, my longtime girlfriend and the person I dedicate this thesis to, for her continued love and support. 


\section{Contents}

List of Figures $\quad$ xi

List of Tables $\quad$ xiii

List of Symbols $\quad$ xiv

List of Acronyms $\quad$ xvi

1 Introduction 1

1.1 MIMO Communication . . . . . . . . . . . . . . . . . . . 1

1.2 Problem Statement and Motivation . . . . . . . . . . . . 4

1.3 Relevant Work . . . . . . . . . . . . . . . 6

1.4 Thesis Contributions .................... 7

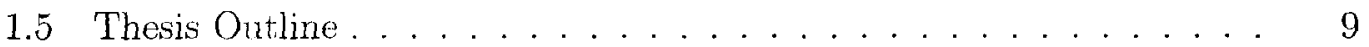

2 Background 11

2.1 MiMO System Model . . . . . . . . . . . . . . . . . . . 11

2.2 Maximum Ratio Combining . . . . . . . . . . . . . 12

2.3 Spatial Modulation . . . . . . . . . . . . . . . . 13

2.3.1 Transmission .................... 14

2.3 .2 Detection (Sib-Optimal) . . . . . . . . . . . 15

2.3 .3 Benefits . . . . . . . . . . . . . . . . 16

viii 
3 Spatial Modulation $\quad 17$

3.1 Detection (Optimal) .................... 17

3.2 Performance Analysis . . . . . . . . . . . . . . . . . . . . . 19

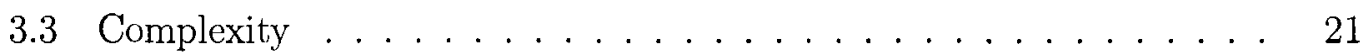

3.4 Simulation Results . . . . . . . . . . . . . . . . 22

3.5 Conclusion ........................... 24

4 Space Shift Keying $\quad 25$

4.1 SSK Modulation . . . . . . . . . . . . . . . . . 26

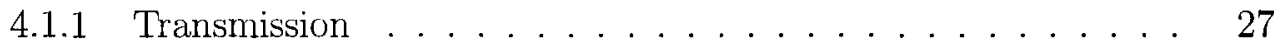

4.1 .2 Detection .......................... 28

4.1 .3 Constellation . . . . . . . . . . . . . . 29

4.2 Performance Analysis . . . . . . . . . . . . . . . . . . . . 30

4.2 .1 Error Probability . . . . . . . . . . . . . . . 31

4.2 .2 Diversity . . . . . . . . . . . . . . . . 33

4.3 Adaptive SSK Transmission . . . . . . . . . . . . . . . 34

4.3.1 Adaptive Symbol Design . . . . . . . . . . . . . 34

4.3 .2 Antenna Selection . . . . . . . . . . . . . 35

4.4 Generalized SSK . . . . . . . . . . . . . . . 36

4.4 Transmission and Detection . . . . . . . . . . . . 37

4.4 .2 Performance Analysis . . . . . . . . . . . . . . . . . 39

4.4 .3 Optimal Constellation Design . . . . . . . . . . . . . 40

4.5 Complexity . . . . . . . . . . . . . . . . . . . 42

4.6 Simulation Results . . . . . . . . . . . . . . . . . . . 43

4.6.1 GSSK versus SM and MRC . . . . . . . . . . . . 44

4.6 .2 SSK versus APM (varying $M$ ) . . . . . . . . . . 46

4.6.3 SSK versus APM (varying $N_{\mathrm{r}}$ ) . . . . . . . . . 47

4.6.4 SSK Adaptive Symbol Design . . . . . . . . . . . . . 48

4.6.5 SSK Antenna Selection . . . . . . . . . . . . . . . 50 
4.6.6 SSK Under Non-ideal Conditions . . . . . . . . . . . . . 50

4.7 Conclusion . . . . . . . . . . . . . . . . . . 54

5 Coded Space Shift Keying $\quad 56$

5.1 Capacity . . . . . . . . . . . . . . . . . . . 57

5.2 SSK Coded Modulation _. . . . . . . . . . . . . . . . . . 61

5.2 .1 Encoding .......................... 61

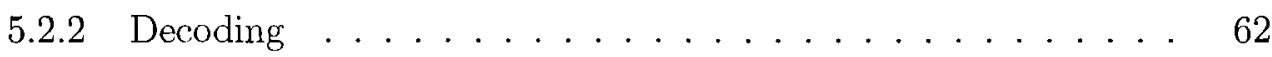

5.3 Coded Performance Analysis . . . . . . . . . . . . . . . 63

5.3 .1 Coded Error Probability . . . . . . . . . . . . . . 63

5.3 .2 Coded Diversity . . . . . . . . . . . . 65

5.4 Simulation Results . . . . . . . . . . . . . . 68

5.4.1 (Quasi-Static) SSK-CM versus APM-CM (varying $M$ ) . . . 68

5.4.2 (Quasi-Static) SSK-CM versus APM-CM (varying $N_{\mathbf{r}}$ ) . . . 69

5.4.3 (Quasi-Static) SSK-TuCM versus APM-TuCM (varying $M$ ) . 70

5.4.4 (Quasi-Static) SSK-TuCM versus APM-TuCM (varying $N_{\mathrm{r}}$ ) · 71

5.4.5 (Fully Interleaved) SSK-CM versus APM-CM (varying $M$ ) . . 72

5.4.6 (Fully Interleaved) SSK-TuCM versus APM-TuCM (varying $M$ ) 74

5.5 Conclusion . . . . . . . . . . . . . . . . 76

6 Conclusion and Future Work $\quad 78$

6.1 Concluding Remarks . . . . . . . . . . . . . . 78

6.2 Future Research . . . . . . . . . . . . . . . . . 79

6.2 .1 Practical implementation issues . . . . . . . . . . 80

6.2 .2 Symbol Design . . . . . . . . . . . . . . . . . 80

6.2.3 Diversity Codebook Design _... . . . . . . . . 81

6.2 .4 UWB Implementation and Analysis . . . . . . . . . . . . . 81

$\begin{array}{ll}\text { Bibliography } & 82\end{array}$ 


\section{List of Figures}

2.1 General MIMO system model. . . . . . . . . . . . . . . . . . . . . 12

2.2 MRC system model. . . . . . . . . . . . . . . . . . . 13

2.3 SM system model. . . . . . . . . . . . . . . . . . . 14

3.1 BER performance of spatial modulation versus SNR, for $m=3 \mathrm{bits} / \mathrm{s} / \mathrm{Hz}$ transmission $\left(N_{1}=4\right) \ldots \ldots \ldots \ldots$

4.1 SSK system model. . . . . . . . . . . . . . . . . . . . . . . . 26

4.2 Illustration of the effective constellation space $\mathcal{X}^{\text {eff }}$. . . . . . . . 30

4.3 GSSK bounds for varying $M, N_{\mathrm{t}}$, and $n_{\mathrm{t}}\left(N_{\mathrm{r}}=2\right) \ldots \ldots \ldots 42$

4.4 BER performance of GSSK versus MRC, and SM, for $m=3 \mathrm{bits} / \mathrm{s} / \mathrm{Hz}$ transmission $\left(N_{\mathrm{r}}=4\right) \ldots \ldots \ldots \ldots . \ldots \ldots$

4.5 BER performance of SSK versus MRC, for varying $M\left(N_{\mathrm{r}}=2\right) . \ldots 46$

4.6 BER performance of SSK versus MRC, for varying $N_{\mathrm{r}}(M=8) . . \quad 47$

4.7 BER performance of SSK with adaptive symbol design, for varying $M$ $\left(N_{1}=1\right) \ldots \ldots \ldots \ldots \ldots \ldots \ldots$

4.8 BER performance of SSK with sub-optimal adaptive symbol design, for varying $M$ and $N_{\mathrm{r}} \ldots \ldots \ldots \ldots \ldots . \ldots \ldots$

4.9 BER performance of SSK with antenna selection, for varying $N_{\mathrm{t}}\left(N_{\mathrm{s}}=\right.$

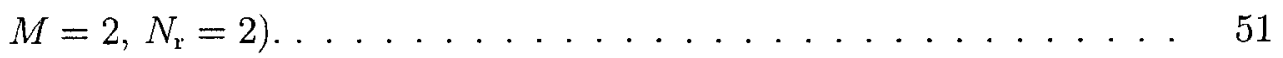

4.10 BER performance of SSK under non-ideal conditions, for varying $M$

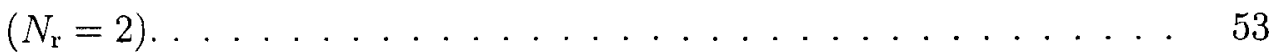

4.11 BER performance of GSSK versus MRC, and SM, for $m=3 \mathrm{bits} / \mathrm{s} / \mathrm{Hz}$ transmission $\left(N_{\mathrm{r}}=4\right)$ under spatial correlation. 
5.1 SSK-CM system model. . . . . . . . . . . . . . . . . . 57

5.2 System model for capacity computation. . . . . . . . . . . . . . 58

5.3 SSK constrained input capacity versus SNR, for varying $M\left(N_{\mathrm{r}}=2\right) . \quad 59$

5.4 SSK constrained input capacity versus SNR, for varying $N_{\mathrm{r}}(M=8) . \quad 60$

5.5 Ilustration of SSK-CM's diversity in quasi-static fading channels. . . . 67

5.6 BER performance of SSK-CM versus PSK-CM, for varying $M\left(N_{\mathrm{r}}=2\right)$ in quasi-static channels. . . . . . . . . . . . . . . . . 69

5.7 BER performance of SSK-CM versus PSK-CM, for varying $N_{\mathrm{r}}(M=8)$ in quasi-static channels. . . . . . . . . . . . . . . 70

5.8 BER performance of SSK-TıCM versus PSK-TıCM, for varying $M$ $\left(N_{\mathrm{r}}=2\right)$ in quasi-static channels. . . . . . . . . . . . 71

5.9 BER performance of SSK-TuCM versus PSK-TuCM, for varying $N_{\mathrm{r}}$ $(M=8)$ in quasi-static channels. . . . . . . . . . 72

$5.10 \mathrm{BER}$ performance of SSK-CM versus PSK-CM, for vaying number of iterations $\left(M=8, N_{\mathrm{r}}=2\right)$ in fully interleaved channels. . . . . . . 73

$5.11 \mathrm{BER}$ performance of SSK-CM versus QAM-CM, for vaying number of iterations $\left(M=16, N_{\mathrm{r}}=2\right)$ in fully interleaved channels. . . . . . 74

5.12 BER performance of SSK-TuCM versus PSK-TuCM, for vaying number of iterations $\left(M=8, N_{\mathrm{r}}=2\right)$ in fully interleaved channels. . . . .

$5.13 \mathrm{BER}$ performance of SSK-TuCM versus QAM-TuCM, for vaying number of iterations $\left(M=16, N_{\mathrm{r}}=2\right)$ in fully interleaved channels. . . . 


\section{List of Tables}

2.1 Example of the SM Mapper Rule. . . . . . . . . . . . . . . 15

4.1 Example of the SSK mapper rule. . . . . . . . . . . . . . 28

4.2 Example of the GSSK Mapper Rule. . . . . . . . . . . . . 38 


\section{List of Symbols}

$\begin{array}{ll}x & \text { the scalar } x \\ |x| & \text { the absolute value of a scalar } \\ x^{*} & \text { the complex conjugate of a scalar } \\ \mathbf{x} & \text { the vector } \mathbf{x} \\ \mathbf{x}^{*} & \text { the complex conjugate of a vector } \\ \mathbf{x}^{T} & \text { the transpose of a vector } \\ \mathbf{x}^{H} & \text { the Hermitian (conjugate transpose) of a vector } \\ \|\mathbf{x}\| & \text { the norm of a vector } \\ \mathbf{X} & \text { the matrix } \mathbf{X} \\ \mathbf{X}^{*} & \text { the complex conjugate of a matrix } \\ \mathbf{X}^{T} & \text { the transpose of a matrix } \\ \mathbf{X}^{H} & \text { the Hermitian (conjugate transpose) of a matrix } \\ \|\mathbf{X}\|_{\mathbf{F}} & \text { the Frobenius norm of a matrix } \\ \approx & \text { approximately equal to } \\ \triangleq & \text { defined as equal to } \\ \exp (x) & \text { the statistical expectation of } A \text { with respect to } \mathbf{x} \\ Q(x) & \left.\text { Ganential of } x \text { (i.e., } e^{x}\right) \\ \left(\begin{array}{l}n \\ k\end{array}\right) & \text { the binomial coefficient (i.e., } n \text { choose } k) \\ P(A) & \text { the probability of an event } A \\ p_{\mathbf{Y}}(y) & E_{\mathbf{x}}[A]\end{array}$




\begin{tabular}{|c|c|}
\hline $\operatorname{Re}\{x\}$ & the real part of a complex variable \\
\hline $\log (x)$ & the natural logarithm (i.e., base $e$ ) \\
\hline $\log _{a}(x)$ & the logarithm with base $a$ \\
\hline $\arg \max _{x} f(x)$ & a value of $x$ that maximizes the function $f(x)$ \\
\hline $\arg \min _{x} f(x)$ & a value of $x$ that minimizes the function $f(x)$ \\
\hline$X \sim p_{X}(x)$ & the random variable $X$ has $\mathrm{PDF} p_{X}(x)$ \\
\hline $\mathbf{I}_{N}$ & the $N \times N$ identity matrix \\
\hline $\mathcal{N}\left(\mathfrak{m}, \frac{\sigma^{2}}{2}\right)$ & $\begin{array}{l}\text { the Gaussian distribution of a random variable, with mean } \mathfrak{m} \text { and vari- } \\
\text { ance } \frac{\sigma^{2}}{2}\end{array}$ \\
\hline $\mathcal{C N}\left(\mathfrak{m}, \sigma^{2}\right)$ & $\begin{array}{l}\text { the complex Gaussian distribution of a random variable, having in- } \\
\text { dependent Gaussian distributed real and imaginary parts (i.e., real } \\
\left.\sim \mathcal{N}\left(\mathfrak{m}, \frac{\sigma^{2}}{2}\right) \text {, imaginary } \sim \mathcal{N}\left(\mathfrak{m}, \frac{\sigma^{2}}{2}\right)\right)\end{array}$ \\
\hline$N_{\mathrm{t}}$ & number of transmit antennas \\
\hline$N_{\mathrm{r}}$ & number of receive antennas \\
\hline $\mathbf{H}$ & MIMO channel matrix \\
\hline $\mathbf{X}$ & transmitted signal \\
\hline $\mathbf{Y}$ & received signal \\
\hline$\eta$ & AWGN noise \\
\hline $\mathcal{X}$ & represents a constellation of size $M$ \\
\hline $\mathcal{X}_{\mathrm{c}}^{\mathrm{k}}$ & $\begin{array}{l}\text { denotes a subset of } \mathcal{X} \text { containing all constellation points with the } k^{\text {th }} \text { bit } \\
\text { equal to } c \in\{0,1\}\end{array}$ \\
\hline$\mu$ & denotes the labeling rule for the constellation $\mathcal{X}$ (i.e., the rule for labeling \\
\hline & bits to a symbol) \\
\hline$\rho$ & denotes the average SNR at each receive antenna \\
\hline$\sigma$ & represents the variance of a random variable \\
\hline
\end{tabular}




\section{List of Acronyms}

AS

APM

AWGN

BER

BICM

BICM-ID

BIOS

BLAST

BPSK

$\mathrm{CM}$

CSI

DIV

GSSK

HYB

IAS

ICI

iid

LLR

LS

MAP

MIMO

ML antenna selection

amplitude/phase modulation

additive white Gaussian noise

bit error rate

bit interleaved coded modulation

bit interleaved coded modulation with iterative decoding

binary input-output symmetric

Bell Laboratories layered space-time

binary phase shift keying

coded modulation

channel state information

diversity

generalized space shift keying

hybrid

inter-antenna synchronization

inter-channel interference

independent and identically distributed

$\log$ likelihood ratio

least squares

maximum a posteriori

multiple-input multiple-output

maximum likelihood 


$\begin{array}{ll}\text { MMSE } & \text { minimum mean-squared error } \\ \text { MRC } & \text { maximum ratio combining } \\ \text { OSIC } & \text { ordered successive interference cancellation } \\ \text { OSTBC } & \text { orthogonal space-time block code } \\ \text { PEP } & \text { pairwise error probability } \\ \text { PDF } & \text { probability density function } \\ \text { PSK } & \text { phase shift keying } \\ \text { QAM } & \text { quadrature amplitude modulation } \\ \text { QPSK } & \text { quadrature phase shift keying } \\ \text { RF } & \text { radio frequency } \\ \text { SC } & \text { spatial correlation } \\ \text { SIMO } & \text { single-input multiple-output } \\ \text { SM } & \text { spatial modulation } \\ \text { SM-OD } & \text { spatial modulation with optimal detection } \\ \text { SMUX } & \text { spatial multiplexing } \\ \text { SNR } & \text { signal to noise ratio } \\ \text { SP } & \text { saddlepoint } \\ \text { SSK } & \text { space shift keying } \\ \text { STBC } & \text { space-time block code } \\ \text { TCM } & \text { trellis-coded modulation } \\ \text { TuCM } & \text { vertical Bell Laboratories layered space-time } \\ \text { V-BLAST } & \end{array}$




\section{Chapter 1}

\section{Introduction}

\subsection{MIMO Communication}

Wireless communication has experienced tremendous growth over the last few years, fueled by increasing consumer demand and breakthrough innovations (e.g., Apple's iPhone [1], a wireless mobile device, sold four million units within six months of release). Most of the world is moving away from tethered devices, and fast becoming one that utilizes the convenience brought upon by wireless technology. Cellular phones, personal digital assistants (PDAs), home networks, cars, and even television sets, to name a few, have adopted some form of wireless communication.

Although wireless communications provide many benefits, there are still many challenges to face. All wireless technologies experience difficulties with signal fading (due to constructive/destructive addition of multi-path signals), interference (e.g.,

co-channels, multiple users), and the limited availability of the frequency spectrum. 
Due to these problems, the data rate, the reliability in transmitted information, and the amount of required processing power are greatly affected. However, the introduction of multiple input multiple output (MIMO) communication systems offer efficient means to overcome many of these challenges.

MIMO technology utilizes multiple antennas at both transmitter and/or receiver terminals in order to achieve certain application needs, without having to increase the amount of bandwidth requirement. These systems fall under three main categories. The first is spatial multiplexing (SMUX), which exploits multiple antenna arrays to transmit more information. One example is the vertical Bell Laboratories layered space-time (V-BLAST) architecture [2], where multiple symbols are multiplexed in space, and transmitted at the same time over all antennas. The second type is diversity (DIV) transmission, where antennas are used to increase the reliability of the message. Similar to channel coding, DIV systems exploit the spatial domain as a coding mechanism to increase reliability (and diversity). The first form of spatial DIV transmission is the well-known Alamouti scheme [3] for two transmit antennas, which exploit antennas to obtain transmit diversity. However, the system diversity is increased at the expense of spectral efficiency, which remains unchanged from a single input multiple output (SIMO) system. This leads to the third type of MIMO systems, termed hybrid (HYB) transmission, which combines both SMUX and DIV schemes. The first application of HYB MIMO transmission is multilayered space-time coding by Tarokh et al. [4], which uses transmit antennas to provide diversity as well as increase spectral efficiency. 
Future applications of MIMO systems is fast growing with a variety of them in the works. For example, certain automotive technology companies, such as DENSO [5] and General Motors [6], are working on vehicule-to-vehicule (V2V) communication. Such communication may be used to prevent accidents, facilitate driving efficiency, and improve road traffic. Another example is wireless high definition (HD) television, where wires would no longer be needed to deliver HD content (for example, Apple TV). Mobile devices, such as Apple's iPhone, are able to deliver high quality web viewing experience, at reasonable speeds, due to $3 \mathrm{G}$ networks, and the use of multiple antennas. With the iPhone, the user is always connected through cellular and wireless fidelity (Wi-Fi) networks, and new application are constantly being added to iPhone's repertory, therefore increasing the demand for high speed data transfer.

MIMO is also at the heart of the IEEE $802.11 \mathrm{n}$ specification for 100Mbps transfer in wireless local area networks (WLANs), providing a much wider range than earlier versions. It is mainly used in Wi-Fi zones, with several available antenna configurations. As well, the IEEE 802.16 standards is focused for the World Inter-operability for Microwave Access (WiMAX) technology, in order to deliver high data rate over long distances. The future of mobile WiMAX is, at the moment, somewhat uncertain since it is not backward compatible with existing cellular technologies, as the case for Wi-Fi. However, with evolving cellular innovations, WiMAX remains a likely candidate to substitute future Wi-Fi implementation. 


\subsection{Problem Statement and Motivation}

MIMO systems provide their own sets of benefits and restraints, but are flexible enough to accommodate different applications. However, some pitfalls that are common amongst MIMO systems, and difficult to avoid, are as follows.

1. Inter-channel interference (ICI): caused by coupling multiple symbols in time and space, maximum likelihood (ML) detection increases exponentially in complexity with the number of transmit antennas. Hence, practical integration of, for example, V-BLAST requires sub-optimal, low complexity receivers [7]. For adequate performance, in most cases, these receivers require the number of receive antennas to be larger or equal to the number of transmit antennas, which is not practical for downlink transmission to small mobile devices. Consequently, avoiding ICI greatly reduces receiver complexity. One example where ICI is avoided is with orthogonal space time block codes (OSTBCs), such as the Alamouti scheme. However, full-rate OSTBCs only exist for two transmit antennas (complex constellations), and eight or less transmit antennas (real constellations) [8]. Therefore, higher transmit diversity is only achieved at the expense of transmission rate.

2. Power Consumption: With multiple signals being transmitted, the processing requirement is larger and more complicated than single antenna systems. This additional processing contributes to delay, and more power consumption. With battery power being a limited but crucial resource in mobile devices, conserving 
power is essential, and difficult to realize under the MIMO framework.

3. Inter-antenna synchronization (IAS): In the BLAST and OSTBC architectures, the detection algorithms assume that all symbols are transmitted at the same time. Hence, IAS is necessary to avoid performance degradation [9], consequently increasing transmitter overhead. For example, Agilent Technologies' signal studio for 3GPP W-CDMA HSPA [10] needs to automatically configure the input and output synchronizing trigger signals when transmit diversity antennas are selected.

4. Radio Frequency (RF) chains: Although multiple antenna elements are relatively inexpensive to deploy, and the digital signal processing requirements are feasible due to increased industry growth, the necessary RF elements are not as simple to implement, [11]. These RF chains are bulky, expensive, and necessary for each antenna that is used. One feasible method around the problem is to employ antenna selection (AS) [11-18] (and references therein).

To exploit MIMO benefits, AS methods can only reduce the number of RF chains to a certain level. Most of the advantages from MIMO communication is due to multiple transmit antennas. For example, in V-BLAST, multiple transmit antennas increase the spectral efficiency of the system without additional bandwidth requirement. For DIV systems, such as OSTBCs, it is multiple transmit antennas that significantly increase the system's diversity. So, although AS 
provides some reduction in RF chains, there is no way around avoiding the increase in RF chains compared to that of a single antenna system. As well, AS generally increases the overhead at the receiver, and is prone to feed back errors when considering transmit AS.

In order to target the aforementioned problems, and gain performance improvements over popular schemes such as V-BLAST and maximum ratio combining (MRC), we present space shift keying (SSK) modulation for MIMO channels.

\subsection{Relevant Work}

Some relevant work that targets the problems mentioned in Section 1.2 are summarized in this section. Not much work has been done related to the following types of systems. Therefore, the available literature is quite scarce.

In $[19,20]$, a scheme entitled SSK is proposed, in which distinct multipath characteristics from different antennas are used to discriminate between transmitted symbols. ${ }^{1}$ The receiver determines which mode of transmission is used (either one antenna or both antennas are activated) in order to detect the message. Chal et al. also mention extensions of their binary scheme to higher order modulation by combining amplitude/phase modulation (APM). However, they only consider the case of two transmit antennas, and their scheme does not avoid ICI and IAS.

Exploiting antenna indices as a source of information is also covered in $[9,21]$,

\footnotetext{
${ }^{1}$ We note that SSK in [19] and in this paper have different meanings. We use the same name because SSK is truly descriptive of the scheme we develop later in Chapter 4.
} 
where precoded sequences of symbols are transmitted using only one antenna. The symbol, in conjunction with the antenna index, is used to decode the message. However, these schemes suffer from lower spectral efficiency due to the employment of parity symbols. Also, only the case of binary/quadrature phase shift keying (BPSK/QPSK) are considered. To overcome the problem of spectral efficiency loss, and in order to generalize to other modulation schemes, spatial modulation (SM) is presented by Mesleh et al. in [22,23]. SM is a pragmatic approach for transmitting information, where the modulator uses well known APM techniques, such as quadrature amplitude modulation (QAM), but also employs the antenna index to convey information. SM is then extended to OFDM in [24], and its performance under nonideal channel conditions (spatial correlation, mutual antenna coupling) is analyzed in $[25]$.

\subsection{Thesis Contributions}

1. We derive the optimal detector for SM, improving over the sub-optimal detection rules suggested in [22]. Thanks to our development, SM compares favorably to other transmission schemes, such as MRC and V-BLAST.

2. In order to support our results, we analyze SM's performance, and derive a closed form expression for the bit error probability when real constellations are used.

3. We introduce SSK, in which only the spatial domain is exploited to modulate 
information. We achieve all of the aforementioned advantages comprising SM, while reducing the transmitter overhead (due to simple symbol modulation), and requiring less detection complexity.

4. SSK's constellation is thoroughly analyzed, where we present the underlying idea that allows SSK to outperform APM schemes. In particular, we show that SSK takes advantage of the fading process by increasing the constellation's dimension, whose points result to be well spread apart. This analysis opens the door to understanding how SM parameters may be chosen to obtain better performance gains (since the trade-off between the number of transmit antennas versus the APM constellation size for SM is chosen heuristically in [23]).

5. We derive tight upper bounds on the bit error rate (BER), and deduce the parameters that need optimization to improve performance.

6. We also present adaptive forms of SSK when transmit channel state information (CSI) is available. In particular, we present adaptive symbol design, as well as antenna selection (AS) in order to improve SSK's performance.

7. As a method of providing SSK with design flexibility, and to reduce the required number of transmit antennas, we present a variant scheme entitled generalized SSK (GSSK). The combination of SSK and GSSK allows for a strong foundation in building practical SM systems, and provides enough flexibility to accommodate different hardware requirements. 
8. We design optimal constellations for GSSK, where it is apparent that tremendous degrees of freedom is available for practical implementation.

9. SSK coded modulation (CM) and bit interleaved CM (BICM) capacity results are also presented, which demonstrate impressive gains over APM-CM techniques.

10. The performance of coded SSK is studied, whereby performance bounds are derived, and illustrate large improvements over APM schemes (especially for quasi-static fading channels). In particular, we discover significant increases in SSK's diversity order compared to that of APM.

11. Extensive simulation results are presented to support our findings, and illustrate the future research potential of SSK modulation.

\subsection{Thesis Outline}

The rest of the thesis is outline as follows.

In Chapter 2, we present some preliminary background knowledge. In particular, we provide the general system model for MIMO communication, as well as present some schemes relevant to this thesis (e.g., MRC, SM).

In Chapter 3, we analyze SM detection, and derive an optimal detector for SM. We also analyze SM performance and complexity, while providing simulation examples to support our results. 
In Chapter 4, we introduce SSK modulation. We present extensive analysis on uncoded SSK transmission, in both non-adaptive and adaptive scenarios.

In Chapter 5, SSK modulation is extended to incorporate channel coding, and we present several analytical results on capacity and performance.

In Chapter 6, concluding remarks are stated, and potential research directions are outlined. 


\section{Chapter 2}

\section{Background}

\subsection{MIMO System Model}

The general system model consists of a MIMO wireless link with $N_{\mathrm{t}}$ transmit and $N_{\mathrm{r}}$ receive antennas, which is shown in Fig. 2.1. A random sequence of independent bits $\mathbf{b}$ enters a space time (ST) encoder, which groups $m$ bits and maps them to a vector $\mathbf{x}=\left[\begin{array}{llll}x_{1} & x_{2} & \cdots & x_{N_{\mathrm{t}}}\end{array}\right]^{T}$ of constellation symbols, where we assume a power constraint of unity (i.e., $E_{\mathbf{x}}\left[\mathbf{x}^{H} \mathbf{x}\right]=1$ ). The signal is transmitted over an $N_{\mathbf{r}} \times N_{\mathbf{t}}$ wireless channel $\mathbf{H}$, and experiences an $N_{\mathbf{r}}$ - dim additive white Gaussian (AWGN) noise $\boldsymbol{\eta}=\left[\begin{array}{llll}\eta_{1} & \eta_{2} & \cdots & \eta_{N_{\mathrm{r}}}\end{array}\right]^{T}$. The received signal is given by

$$
\mathbf{y}=\sqrt{\rho} \mathbf{H} \mathbf{x}+\boldsymbol{\eta}
$$


where $\rho$ is the average SNR at each receive antenna, and $\mathbf{H}$ and $\boldsymbol{\eta}$ have independent and identically distributed (iid) entries according to $\mathcal{C N}(0,1)$. The received signal is then passed to a ST decoder, where an estimate of the transmitted bits $\hat{\mathbf{b}}$ is obtained.

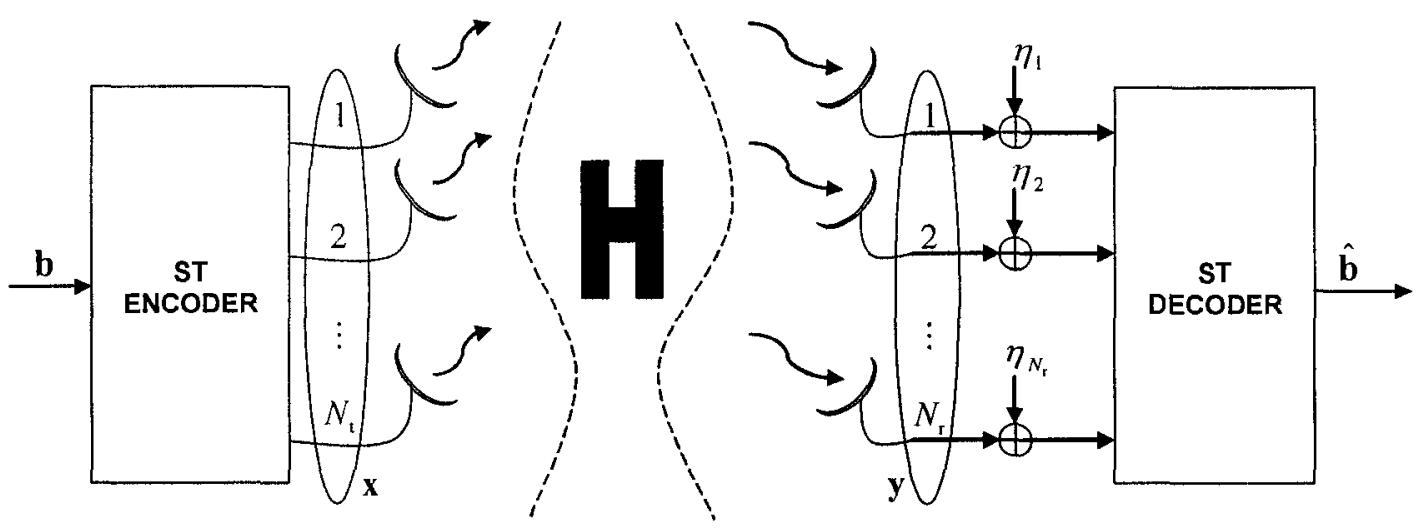

Figure 2.1: General MIMO system model.

\subsection{Maximum Ratio Combining}

MRC is used for SIMO channels that provide an $L^{\text {th }}$ order of diversity. The general system model is shown in Fig. 2.2. The model is similar to Fig. 2.1, but with $N_{\mathrm{t}}=1$, $N_{\mathrm{r}}=L$, and where we replace the ST encoder and ST decoder with an APM mapper and a MRC receiver, respectively. The APM mapper takes $m=\log _{2}(M)$ bits and maps them to an APM constellation symbol $x_{j}$, obtained from $\mathcal{X}$.

The received signal is therefore given by $\mathbf{y}=\sqrt{\rho} \mathbf{h} x_{j}+\boldsymbol{\eta}$, where $\mathbf{h}$ denotes a column of H. Assuming perfect CSI and equally likely inputs, the optimal detector is ML, 


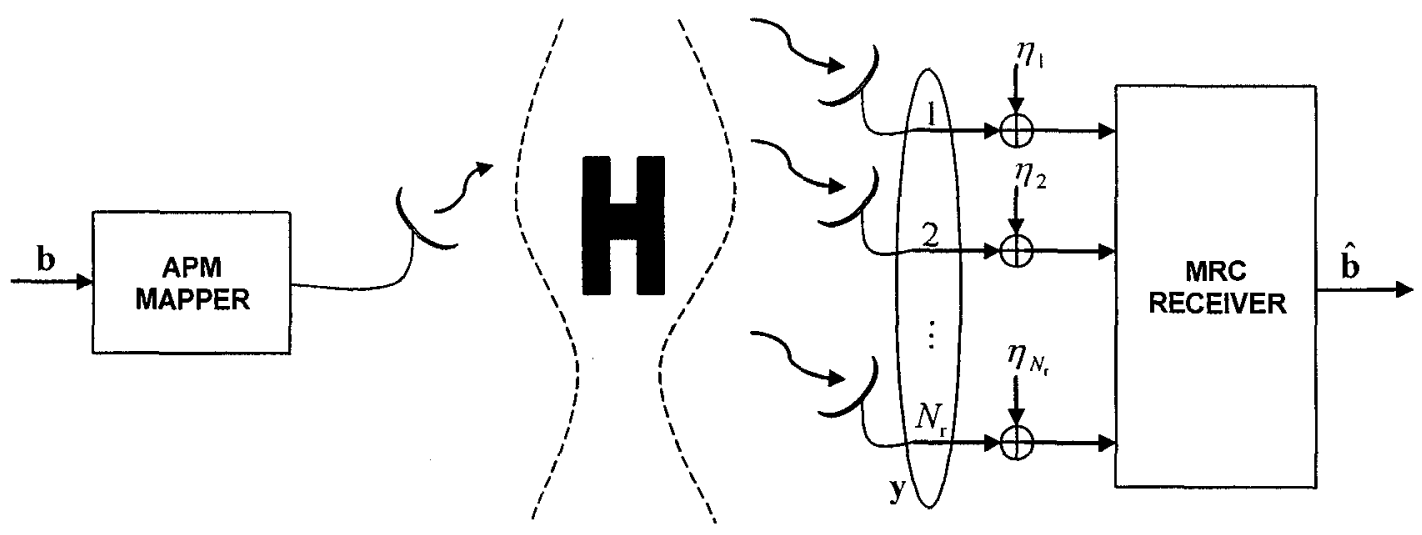

Figure 2.2: MRC system model.

and given by [26, Ch. 2]

$$
\begin{aligned}
x_{\hat{\jmath}} & =\underset{x_{j}}{\arg \max } p_{\mathbf{Y}}\left(\mathbf{y} \mid x_{j}, \mathbf{H}\right) \\
& =\underset{x_{j}}{\arg \max } \sqrt{\rho}\left[\operatorname{Re}\left\{\left(\mathbf{h} x_{j}\right)^{H} \mathbf{y}\right\}-\frac{1}{2}\left\|\mathbf{h} x_{j}\right\|_{\mathbf{F}}^{2}\right],
\end{aligned}
$$

where $p_{\mathbf{Y}}\left(\mathbf{y} \mid \mathbf{x}_{j q}, \mathbf{H}\right)=\pi^{-N_{\mathrm{r}}} \exp \left(-\left\|\mathbf{y}-\sqrt{\rho} \mathbf{h} x_{j}\right\|_{\mathrm{F}}^{2}\right)$. Equation (2.2) is referred to as the MRC rule.

\subsection{Spatial Modulation}

Spatial modulation (SM) is introduced by Mesleh et al. $[22,23]$ in an effort to remove ICI, and the need for precise time synchronization amongst antennas. SM is a pragmatic approach for transmitting information, where the modulator uses well known APM techniques (e.g., PSK, QAM), but also employs the antenna index to convey information. Ideally, only one antenna remains active during transmission so that 
ICI is avoided ${ }^{1}$, and IAS is no longer needed (as in the case of V-BLAST, where all antennas transmit at the same time).

\subsubsection{Transmission}

The general SM system model is shown in Fig. 2.3. The SM mapper groups $m=$ $\log _{2}\left(M N_{\mathrm{t}}\right)$ bits and maps them to a constellation vector $\mathbf{x}_{j q}$ of the form

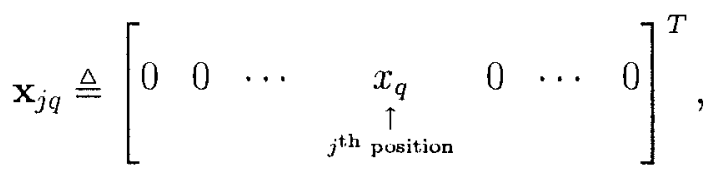

where $j$ represents the activated antenna, and $x_{q}$ is the $q^{\text {th }}$ symbol from the $M$-ary constellation $\mathcal{X}$. In $\mathrm{SM}$, only one antenna remains active during transmission and hence, only one symbol in $\mathbf{x}$ is nonzero (i.e., only the $j^{\text {th }}$ antenna remains active during symbol transmission).

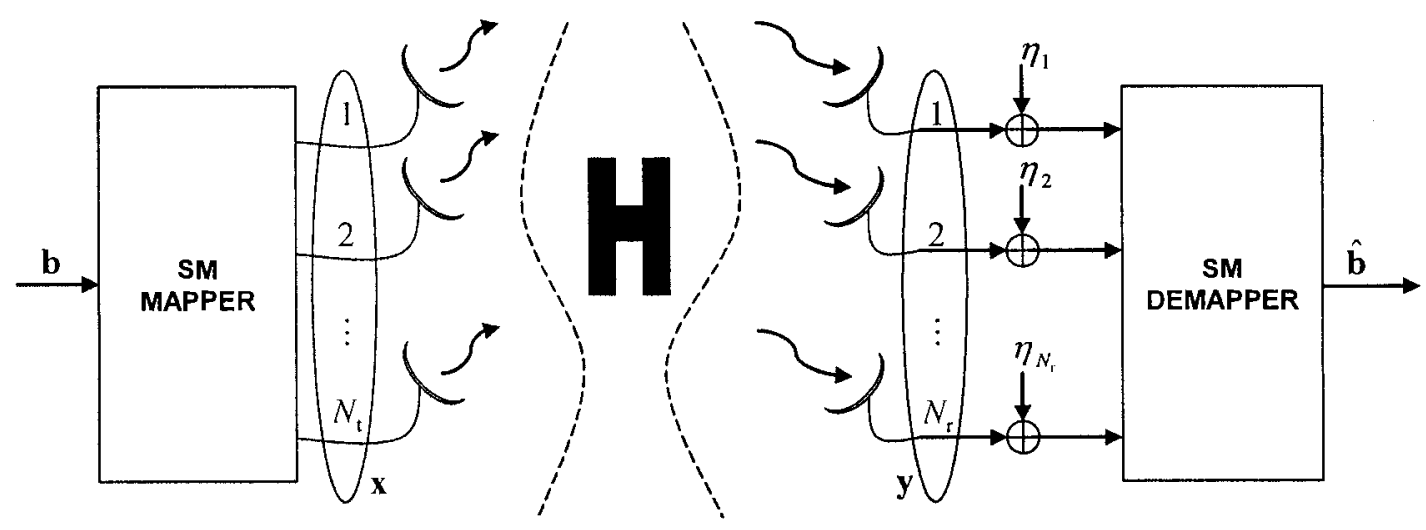

Figure 2.3: SM system model.

\footnotetext{
${ }^{1}$ Assuming the pulse shaping period does not overlap amongst antennas.
} 
Table 2.1 illustrates an example of the mapper for $3 \mathrm{bits} / \mathrm{s} / \mathrm{Hz}$ transmission $\left(N_{\mathrm{t}}=\right.$ 4, $M=2$ ). Each information bit is mapped to a BPSK symbol, and transmitted on one of the four antennas. The output of the channel when $x_{q}$ is transmitted from the $j^{\text {th }}$ antenna is expressed as

$$
\mathbf{y}=\sqrt{\rho} \mathbf{h}_{j} x_{q}+\boldsymbol{\eta},
$$

where $\mathbf{h}_{j}$ denotes the $j^{\text {th }}$ column of $\mathbf{H}$.

Table 2.1: Example of the SM Mapper Rule.

\begin{tabular}{|c|c|c|c|c|}
\hline & b & $x$ & antenna $j$ & $\mathrm{x}$ \\
\hline$[0$ & $\begin{array}{ll}0 & 0 \\
\end{array}$ & +1 & 1 & {$\left[\begin{array}{llll}1 & 0 & 0 & 0\end{array}\right]^{T}$} \\
\hline$[0$ & $\left.\begin{array}{ll}0 & 1\end{array}\right]$ & -1 & 1 & {$\left[\begin{array}{llll}-1 & 0 & 0 & 0\end{array}\right]^{T}$} \\
\hline$[0$ & $\begin{array}{ll}1 & 0\end{array}$ & +1 & 2 & {$\left[\begin{array}{llll}0 & 1 & 0 & 0\end{array}\right]^{\frac{T}{T}}$} \\
\hline$[0$ & $\left.\begin{array}{ll}1 & 1\end{array}\right]$ & -1 & 2 & {$\left[\begin{array}{llll}0 & -1 & 0 & 0\end{array}\right]^{T}$} \\
\hline 1 & $\begin{array}{ll}0 & 0\end{array}$ & +1 & 3 & {$\left[\begin{array}{llll}0 & 0 & 1 & 0\end{array}\right]^{\frac{1}{T}}$} \\
\hline$[1$ & $\left.\begin{array}{ll}0 & 1\end{array}\right]$ & -1 & 3 & {$\left[\begin{array}{llll}0 & 0 & -1 & 0\end{array}\right]^{T}$} \\
\hline 1 & $\left.\begin{array}{ll}1 & 0\end{array}\right]$ & +1 & 4 & {$\left[\begin{array}{llll}0 & 0 & 0 & 1\end{array}\right]^{\frac{T}{T}}$} \\
\hline$[1$ & $\left.\begin{array}{ll}1 & 1\end{array}\right]$ & -1 & 4 & {$\left[\begin{array}{llll}0 & 0 & 0 & -1\end{array}\right]^{T}$} \\
\hline
\end{tabular}

\subsubsection{Detection (Sub-Optimal)}

In [22], a sub-optimal detection rule somewhat based on MRC is given by

$$
\begin{gathered}
\hat{\jmath}=\underset{j}{\arg \max } z_{j} \\
\hat{q}=\mathcal{D}\left(z_{\hat{\jmath}}\right),
\end{gathered}
$$


where $z_{j}=\frac{\left|\mathbf{h}_{j}^{H} \mathbf{y}\right|}{\left\|\mathbf{h}_{j}\right\|_{F}^{2}}, \hat{\jmath}$ and $\hat{q}$ represent the estimated antenna and symbol index, respectively, and $\mathcal{D}$ is the constellation demodulator function. Since the mapping is one to one, the demapper obtains an estimate of the transmitted bits by taking $\hat{\jmath}$ and $\hat{q}$ as inputs.

\subsubsection{Benefits}

In [22] SM is shown to perform similar to V-BLAST, but with lower computational complexity. The complexity reduction is due to SM's simple detection, a consequence of avoiding ICI. As well, IAS is avoided, and ideally, only one RF chain is necessary. In the next, chapter, we study SM in more detail, and present an optimal detection method. 


\section{Chapter 3}

\section{Spatial Modulation}

In this chapter, we present a more detailed analysis of SM [27]. In particular, we derive a new SM optimal detector (SM-OD), which does not suffer from the restrictions of Mesleh's original detector. Consequently, with the new detector, SM's full potential is demonstrated. As well, an in depth analysis of SM-OD's performance and complexity is also presented.

\subsection{Detection (Optimal)}

The simulation results of [22] could not be reproduced using the conventional channel assumptions of Section 2.1. The reason for this can be seen (in the noiseless case) by substituting (2.3) for $\mathbf{y}$ in (2.4). Therefore, $z_{j}$ reduces to $\frac{\sqrt{P}\left|\mathbf{h}_{k}^{H} \mathbf{h}_{j} x_{q}\right|}{\left\|\mathbf{h}_{k}\right\|_{\mathbf{F}}^{2}}$ and, in order to detect the correct antenna index (i.e., $k=j$ ), we require $\frac{\left|\mathbf{h}_{k}^{H} \mathbf{h}_{j}\right|}{\left\|\mathbf{h}_{k}\right\|_{\mathrm{F}}^{2}}<1$. By invoking 
Cauchy's inequality to the left hand side, we find that $\left\|\mathbf{h}_{j}\right\|_{\mathrm{F}} \leq\left\|\mathbf{h}_{k}\right\|_{\mathrm{F}}$ is a necessary condition for antenna detection without errors, which should materialize in the absence of noise. One way to ensure this condition is by normalizing the channel prior to transmission (i.e., $\left\|\mathbf{h}_{j}\right\|_{\mathrm{F}}^{2}=c$ for all $j$, where $c$ is a constant). We refer to these channels as constrained. We now derive SM-OD, where the need for constrained channels is not necessary.

Since the channel inputs are assumed equally likely, the optimal detector is based on the ML principle:

$$
\begin{aligned}
{\left[\hat{\jmath}_{\mathrm{ML}}, \hat{q}_{\mathrm{ML}}\right] } & =\underset{j, q}{\arg \max } p_{\mathbf{Y}}\left(\mathbf{y} \mid \mathbf{x}_{j q}, \mathbf{H}\right) \\
& =\underset{j, q}{\arg \min } \sqrt{\rho}\left\|\mathbf{g}_{j q}\right\|_{\mathbf{F}}^{2}-2 \operatorname{Re}\left\{\mathbf{y}^{H} \mathbf{g}_{j q}\right\}
\end{aligned}
$$

where $\mathbf{g}_{j q}=\mathbf{h}_{j} x_{q}, 1 \leq j \leq N_{\mathrm{t}}, 1 \leq q \leq M$, and

$$
p_{\mathbf{Y}}\left(\mathbf{y} \mid \mathbf{x}_{j q}, \mathbf{H}\right)=\pi^{-N_{\mathbf{r}}} \exp \left(-\left\|\mathbf{y}-\sqrt{\rho} \mathbf{H} \mathbf{x}_{j q}\right\|_{\mathrm{F}}^{2}\right)
$$

is the PDF of $\mathbf{y}$, conditioned on $\mathbf{x}_{j q}$ and $\mathbf{H}$. It can be seen that optimal detection requires a joint detection of the antenna indices and symbols, as opposed to the scheme outlined in Section 2.3.2, where the problem is decoupled. 


\subsection{Performance Analysis}

The performance of the SM-OD is derived using the well known union bounding technique $[28$, p. 261-262]. The average BER in SM is bounded as

$$
\begin{aligned}
P_{\mathrm{e}, \mathrm{bit}}^{\mathrm{SM}} & \leq E_{\mathbf{x}}\left[\sum_{\hat{\jmath}, \hat{q}} N_{\mathrm{SM}}(q, \hat{q}) P_{\mathrm{SM}}\left(\mathbf{x}_{j q} \rightarrow \mathbf{x}_{\hat{\jmath} \hat{q}}\right)\right] \\
& =\sum_{j=1}^{N_{\mathrm{t}}} \sum_{q=1}^{M} \sum_{\hat{\jmath}=1}^{N_{\mathrm{t}}} \sum_{\hat{q}=1}^{M} \frac{N(q, \hat{q}) P\left(\mathbf{x}_{j q} \rightarrow \mathbf{x}_{\hat{j} \hat{q}}\right)}{N_{\mathrm{t}} M}
\end{aligned}
$$

where $N_{\mathrm{SM}}(q, \hat{q})$ is the number of bits in error between the symbol $x_{q}$ and $x_{\hat{q}}$, and $P_{\mathrm{SM}}\left(\mathbf{x}_{j q} \rightarrow \mathbf{x}_{\hat{\jmath} \hat{q}}\right)$ denotes the pairwise error probability (PEP) of deciding on the constellation vector $\mathbf{x}_{\hat{\jmath} \hat{q}}$ given that $\mathbf{x}_{j q}$ is transmitted. By using (3.1), the PEP conditioned on $\mathbf{H}$ is obtained as

$$
P_{\mathrm{SM}}\left(\mathbf{x}_{j q} \rightarrow \mathbf{x}_{\hat{\jmath} \hat{q}} \mid \mathbf{H}\right)=P_{\mathrm{SM}}\left(d_{j q}>d_{\hat{\jmath} \hat{q}} \mid \mathbf{H}\right)=Q\left(\sqrt{\kappa_{\mathrm{SM}}}\right)
$$

where $d_{j q}=\left(\sqrt{\rho}\left\|\mathbf{g}_{j q}\right\|_{\mathrm{F}}^{2}-2 \operatorname{Re}\left\{\mathbf{y}^{H} \mathbf{g}_{j q}\right\}\right)$, and

$$
Q(x)=\int_{x}^{\infty} \frac{1}{2 \pi} e^{-\frac{t^{2}}{2}} d t
$$

We define $\kappa_{\mathrm{SM}}$ as

$$
\kappa_{\mathrm{SM}} \triangleq \frac{\varrho}{2}\left\|\mathbf{g}_{j q}-\mathbf{g}_{j \hat{q}}\right\|_{\mathrm{F}}^{2}=\sum_{n=1}^{N_{\mathrm{r}}}|A(n)+i B(n)|^{2},
$$


where $i=\sqrt{-1}$ and

$$
\begin{aligned}
& A(n)=\sqrt{\frac{\rho}{2}}\left(h_{n j}^{R} x_{q}^{R}-h_{n j}^{I} x_{q}^{I}-h_{n \hat{\jmath}}^{R} x_{\hat{q}}^{R}+h_{n \hat{\jmath}}^{I} x_{\hat{q}}^{I}\right) \\
& B(n)=\sqrt{\frac{\rho}{2}}\left(h_{n j}^{R} x_{q}^{I}+h_{n j}^{I} x_{q}^{R}-h_{n \hat{\jmath}}^{R} x_{\hat{q}}^{I}-h_{n \hat{\jmath}}^{I} x_{\hat{q}}^{R}\right) .
\end{aligned}
$$

The superscript $R$ and $I$ denote the real and imaginary part, respectively, and $h_{n j}$ is the element of $\mathbf{H}$ in the $n^{\text {th }}$ row, and $j^{\text {th }}$ column. The distribution of the random variable $\kappa$ in (3.4) is not easily obtained since $A(n)$ and $B(n)$ are not, in general, independent. In this case, the performance can be evaluated numerically. However, for symbols $x$ drawn from a real constellation $\mathcal{X}$, this independence is satisfied and (3.4) reduces to $\kappa_{\mathrm{SM}}=\sum_{n=1}^{2 N_{\mathrm{v}}} \alpha_{\mathrm{SM}}^{2}(n)$ where $\alpha_{\mathrm{SM}}(n) \sim \mathcal{N}\left(0, \sigma_{\mathrm{SM}}^{2}\right)$ with $\sigma_{\mathrm{SM}}^{2}=\frac{\rho\left(\left|x_{q}\right|^{2}+\left|x_{\hat{q}}\right|^{2}\right)}{4}$. Hence, $\kappa_{\mathrm{SM}}$ is a chi-squared random variable with $2 N_{\mathrm{r}}$ degrees of freedom and PDF $p_{\kappa}(v)$ given by $[28$, p. 41$]$

$$
p_{\kappa}(v)=\frac{v^{\frac{s}{2}-1} \exp \left(-\frac{v}{2 \sigma^{2}}\right)}{\left(2 \sigma^{2}\right)^{\frac{s}{2}} \Gamma\left(\frac{s}{2}\right)}, v>0
$$

where $\sigma^{2}=\sigma_{\mathrm{SM}}^{2}$. The PEP can then be formulated as

$$
P_{\mathrm{SM}}\left(\mathbf{x}_{j q} \rightarrow \mathbf{x}_{\hat{\jmath} \hat{q}}\right)=\int_{v=0}^{\infty} Q(\sqrt{v}) p_{\kappa}(v) d v
$$


which has a closed form expression given in [29, Eq. (64)]. Thus,

$$
P_{\mathrm{SM}}\left(\mathbf{x}_{j q} \rightarrow \mathbf{x}_{\hat{\jmath} \hat{q}}\right)=\gamma_{\mathrm{SM}}^{N_{\mathrm{r}}} \sum_{k=0}^{N_{\mathrm{r}}-1}\left(\begin{array}{c}
N_{\mathrm{r}}-1+k \\
k
\end{array}\right)\left[1-\gamma_{\mathrm{SM}}\right]^{k}
$$

where $\gamma_{\mathrm{SM}}=\frac{1}{2}\left(1-\sqrt{\frac{\sigma_{\mathrm{SM}}^{2}}{1+\sigma_{\mathrm{SM}}^{2}}}\right)$. Pligging in (3.6) into (3.2), we obtain

$$
P_{\mathrm{e}, \mathrm{bit}}^{\mathrm{SM}} \leq \sum_{q=1}^{M} \sum_{\hat{q}=1}^{M} \frac{N_{\mathrm{t}} N_{\mathrm{SM}}(q, \hat{q}) \gamma_{\mathrm{SM}}^{N_{\mathrm{r}}} \sum_{k=0}^{N_{\mathrm{r}}-1}\left(\begin{array}{c}
N_{x}-1+k \\
k
\end{array}\right)\left[1-\gamma_{\mathrm{SM}}\right]^{k}}{M}
$$

\subsection{Complexity}

To compare the complexity of SM-OD and SM, we use the number of multiplications required in the detection process as a metric. As for the number of additions, it can be shown to have a similar value for both detectors. The complexity of Mesleh's SM detector is obtained from [22] as

$$
\delta_{\mathrm{SM}}=2 N_{\mathrm{r}} N_{\mathrm{t}}+N_{\mathrm{t}}+f(M)
$$

where the last term depends on the type of demodulation assumed. ${ }^{1}$

Similar to [22], we analyze (3.1) to obtain the complexity of SM-OD. It, can be shown that the first and second terms in (3.1) result in $N_{\mathrm{r}} N_{\mathrm{t}}+M$ and $N_{\mathrm{r}} N_{\mathrm{t}}+N_{\mathrm{t}} M$ multiplications, respectively (for the first term, we simplified the computation by

\footnotetext{
${ }^{1}$ In [22], the complex multiplications involved with $\left\|\mathbf{h}_{j}\right\|_{\mathrm{F}}^{2}$ and $|\cdot|$ are ignored, and results in a slightly different $\delta_{\mathrm{SM}}$.
} 
splitting $\left.\left\|\mathbf{h}_{j} x_{q}\right\|_{\mathrm{F}}^{2}=\left\|\mathbf{h}_{j}\right\|_{\mathrm{F}}^{2}\left|x_{q}\right|^{2}\right)$. Hence, the total computational complexity for SMOD is given by

$$
\delta_{\mathrm{SM}-\mathrm{OD}}=2 N_{\mathrm{r}} N_{\mathrm{t}}+N_{\mathrm{t}} M+M
$$

We observe that the complexity involved between both detectors is comparable. These results motivate the use of SM-OD in order to fully exploit SM's advantages.

\subsection{Simulation Results}

In this section, we present some examples to compare SM-OD and Mesleh's SM detection scheme [22]. We perform Monte Carlo simulations for $10^{6}$ channel realizations and plot the average BER performance versus $\rho$. We target $m=3 \mathrm{bits} / \mathrm{s} / \mathrm{Hz}$ transmission with $N_{\mathrm{r}}=4$ antennas. Figure 3.1 illustrates the simulation results for both constrained (dotted line) and conventional (solid line) channel assumptions (Section $3.1)$.

For reference, we use two different transmission setups. The first one is APM, 8QAM transmission with $N_{\mathrm{t}}=1$ (single antenna transmission) and $M=8$. The second is V-BLAST with BPSK modulation, $N_{\mathrm{t}}=3$, and ordered successive interference cancellation (OSIC) with the minimum mean squared error (MMSE) receiver [7]. SM with BPSK and $N_{\mathrm{t}}=4$ antennas is shown for both sub-optimal [22] and optimal receivers. We also plot the $P_{\mathrm{e}, \mathrm{bit}}^{\mathrm{SM}}$ bound of (3.7) for SM using BPSK modulation, where $N_{\mathrm{t}}=4, M=2, \sigma_{\mathrm{SM}}^{2}=\frac{\rho}{2}$ and $N_{\mathrm{SM}}(q, \hat{q})=\left\{\begin{array}{cc}0, & q=\hat{q} \\ 1, & q \neq \hat{q}\end{array}\right.$. 


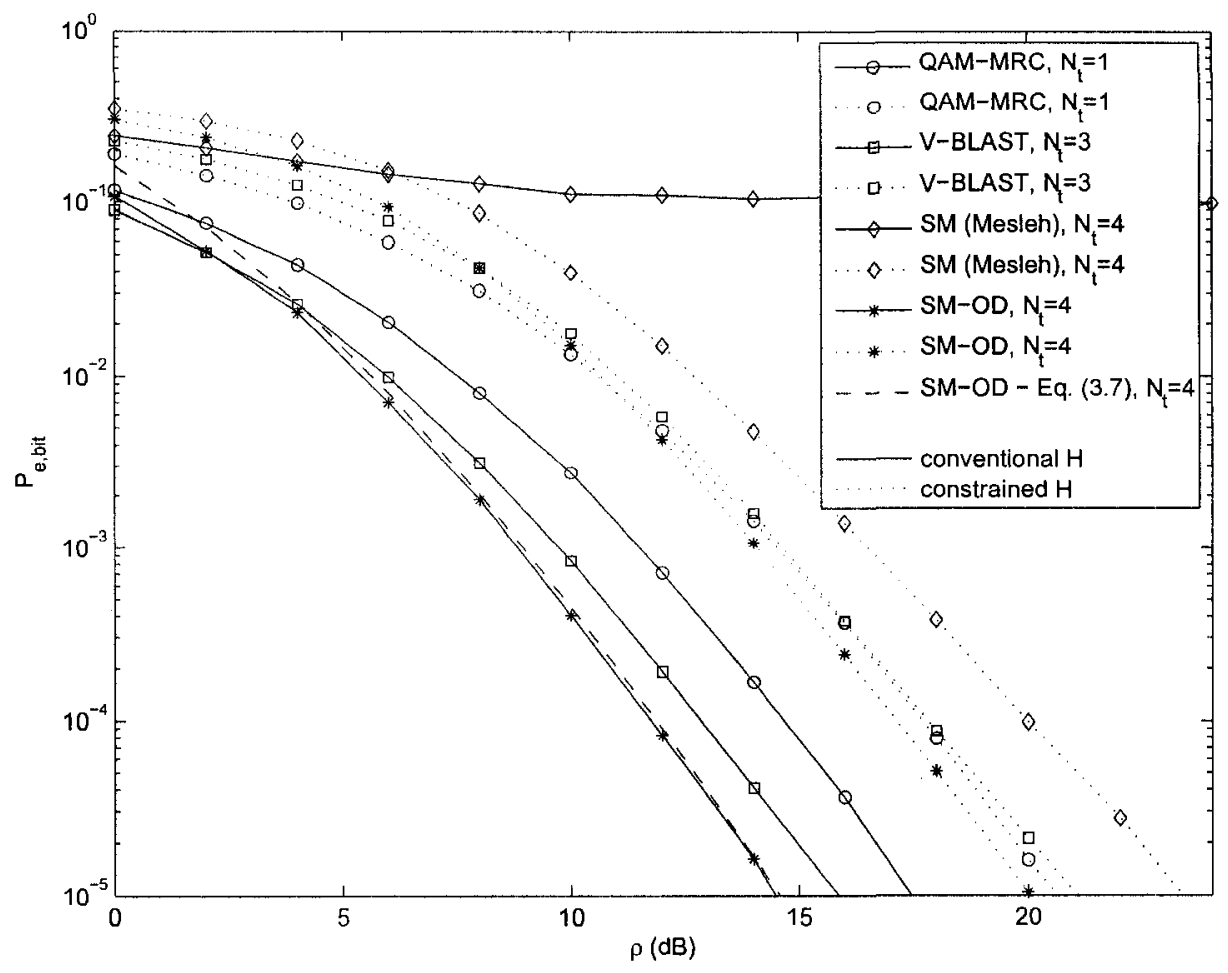

Figure 3.1: BER performance of spatial modulation versus SNR, for $m=3 \mathrm{bits} / \mathrm{s} / \mathrm{Hz}$ transmission $\left(N_{\mathrm{r}}=4\right)$.

Let us first consider the case of constrained channels (dotted lines). As shown, SM-OD gains $4 \mathrm{~dB}$ at $P_{\mathrm{e}, \mathrm{bit}}=10^{-5}$ over SM. Also, (minor) gains are also evident over MRC and V-BLAST, which is not the case with sub-optimal detection. For the conventional channel model (solid line), it is shown that SM-OD provides performance improvements of $3 \mathrm{~dB}$ over APM, and $1 \mathrm{~dB}$ over V-BLAST (at $P_{\mathrm{e}, \mathrm{bit}}=10^{-5}$ ). As well, we notice that the derived BER bounds are relatively tight, and support our simulation results. 


\subsection{Conclusion}

In this chapter, we derived the optimal detector for SM, which does not suffer from the assumptions of the original detector. As well, the optimal detector performs significantly better (at similar computational complexity), and our results were supported by theoretical BER analysis.

Next, we introduce SSK, a fundamental component of SM providing similar performance with lower complexity requirements. 


\section{Chapter 4}

\section{Space Shift Keying}

In this chapter we present SSK modulation [30]. In SSK, antenna indices are used as the only means to relay information, which makes it somewhat a subset of SM. However, this elimination of APM provides SSK with notable differences and advantages over SM:

1. Detection complexity is lowered, while the performance is almost identical to SM-OD.

2. Because phase and amplitude of the pulse do not convey information, transceiver requirements are less stringent than for APM (e.g., non-coherent detectors may be considered).

3. The simplicity of SSK's framework provides ease of integration within communication systems. For example, one envisioned application is ultra wide band (UWB), where it is pulses that are used rather than APM signals. 
All of this, as well as SM's inherent advantages, motivates our presentation of this simple modulation technique.

\subsection{SSK Modulation}

The general system model is shown in Fig. 4.1. The model is similar to Fig. 2.1, where we replace the ST encoder and ST decoder with an SSK mapper and SSK detector, respectively. In SSK, only one antenna remains active during transmission and hence, only one $x_{j}, j=1, \ldots, N_{\mathrm{t}}$, in $\mathbf{x}$ is nonzero. Therefore, ideally only one $\mathrm{RF}$ chain is required. But, due to pulse shaping, the transmitted pulse will extend a couple of symbol periods, and restrict the RF chain from being switched to another antenna. ${ }^{1}$ However, since pulse shaping is not required in the UWB framework, the cost of RF chains is fixed in that case, regardless of the number of antennas.

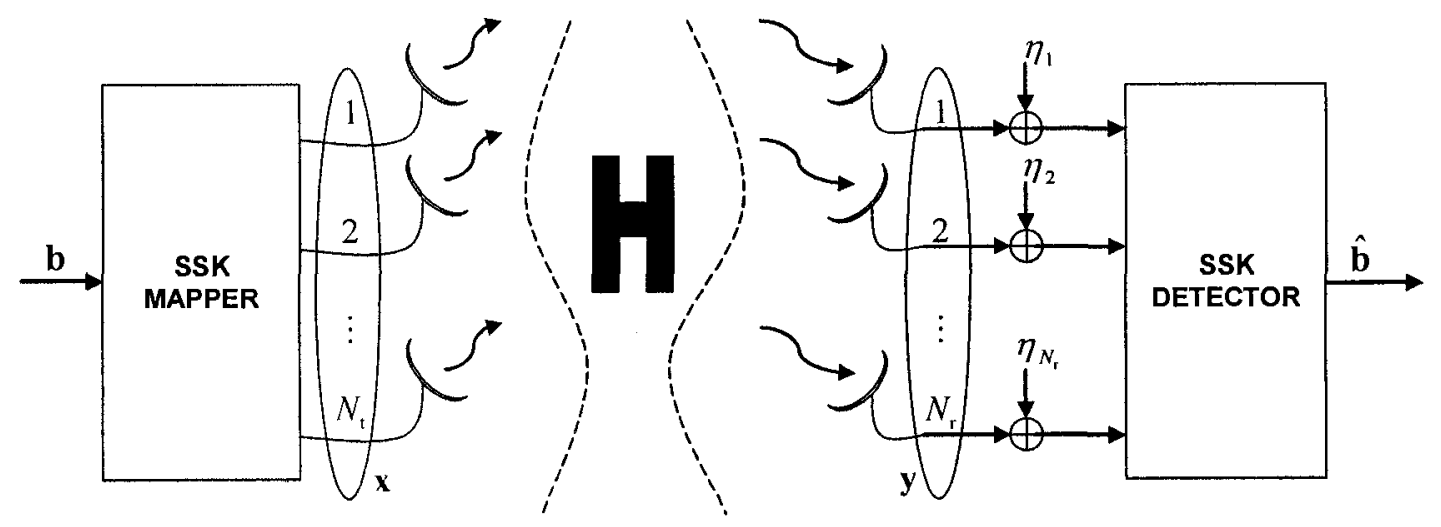

Figure 4.1: SSK system model.

\footnotetext{
${ }^{1}$ Therefore, at the most, we would require the number of $\mathrm{RF}$ chains to equal the number of symbol period durations in the transmitted pulse.
} 


\subsubsection{Transmission}

SSK modulation consists of groups of $m=\log _{2}(M)$ bits that are mapped to a symbol $x_{j}$, which is then transmitted from the $j^{\text {th }}$ antenna. We note that, although the symbol itself does not contain information, it might be designed to optimize transmission. For now, we use $x_{j}=1$ for all $j$, and consider symbol design with limited feedback in Section 4.3. Even though $x_{j}$ does not convey information, its location in $\mathbf{x}$ does. The vector $\mathbf{x}$ specifies the activated antenna, during which all other antennas remain idle, and has the following form:

$$
\mathbf{x}_{j} \triangleq\left[\begin{array}{ccccccc}
0 & 0 & \cdots & \underset{\substack{\uparrow \\
j^{\text {th }} \text { pusition }}}{1} & & &
\end{array}\right]^{T}
$$

Hence, the channel output is given by

$$
\mathbf{y}=\sqrt{\rho} \mathbf{h}_{j}+\eta
$$

when the $j^{\text {th }}$ antenna is used.

Remark 1 Only one column of $\mathbf{H}$ (i.e. $\mathbf{h}_{j}$ ) is activated, and the column changes depending on the transmitted symbol. Essentially, these columns act as random constellation points for SSK modulation.

In $M$-ary SSK modulation (i.e., where $\log _{2}(M)$ bits are transmitted per channel use), $N_{\mathrm{t}}$ must equal $M$. In the case of hardware constraints on the number of transmit antennas, GSSK may be employed to reduce antenna requirements (Section 4.4). An 
example of 4-ary SSK modulation is given in Table 4.1.

Table 4.1: Example of the SSK mapper rule.

\begin{tabular}{c|c|c|c}
$\mathbf{b}=\left[\begin{array}{ll}b_{1} & b_{2}\end{array}\right]$ & symbol & antenna index $j$ & $\mathbf{x}=\left[\begin{array}{ccc}x_{1} & \cdots & x_{4}\end{array}\right]^{T}$ \\
\hline$\left[\begin{array}{ll}0 & 0\end{array}\right]$ & 0 & 1 & {$\left[\begin{array}{llll}1 & 0 & 0 & 0\end{array}\right]^{T}$} \\
{$\left[\begin{array}{ll}0 & 1\end{array}\right]$} & 1 & 2 & {$\left[\begin{array}{llll}0 & 1 & 0 & 0\end{array}\right]^{T}$} \\
{$\left[\begin{array}{ll}1 & 0\end{array}\right]$} & 2 & 3 & {$\left[\begin{array}{llll}0 & 0 & 1 & 0\end{array}\right]^{T}$} \\
{$\left[\begin{array}{ll}1 & 1\end{array}\right]$} & 3 & 4 & {$\left[\begin{array}{llll}0 & 0 & 0 & 1\end{array}\right]^{T}$}
\end{tabular}

\subsubsection{Detection}

The detector's main function is to obtain the antenna index used at the transmitter.

Since the channel inputs are assumed equally likely, the optimal detector is ML, which is given by

$$
\begin{aligned}
\hat{\jmath} & =\underset{j}{\arg \max } p_{\mathbf{Y}}\left(\mathbf{y} \mid \mathbf{x}_{j}, \mathbf{H}\right) \\
& =\underset{j}{\arg \min } \| \mathbf{y}-\left.\sqrt{\rho} \mathbf{h}_{j}\right|_{\mathrm{F}} ^{2} \\
& =\underset{j}{\arg \max } \operatorname{Re}\left\{\left(\mathbf{y}-\frac{\sqrt{\sqrt{ }}}{2} \mathbf{h}_{j}\right)^{H} \mathbf{h}_{j}\right\},
\end{aligned}
$$

where $\hat{\jmath}$ represents the estimated antenna index, $1 \leq j \leq N_{\mathbf{t}}$, and $p_{\mathbf{Y}}\left(\mathbf{y} \mid \mathbf{x}_{j}, \mathbf{H}\right)$ is given by

$$
p_{\mathbf{Y}}\left(\mathbf{y} \mid \mathbf{x}_{j}, \mathbf{H}\right)=\frac{\exp \left(-\left\|\mathbf{y}-\sqrt{\rho} \mathbf{H} \mathbf{x}_{j}\right\|_{\mathrm{F}}^{2}\right)}{\pi^{N_{r}}}
$$




\subsubsection{Constellation}

We now look into SSK's constellation in more detail, and highlight some of its strength. Consider a fixed channel realization $\mathrm{H}$, and the effective $N_{\mathrm{r}}$ - dim constellation symbol

$$
\mathbf{x}_{j, \mathrm{eff}}=\mathbf{H x}_{j}
$$

shown in Fig. 4.2 for both APM and SSK. In APM, the effective constellation $\mathcal{X}^{\text {eff }}$ is composed of scaled versions of the vector $\mathbf{h} x_{j}$. But in SSK, $\mathcal{X}^{\text {eff }}$ is made up of scaled versions of all columns of $\mathbf{H}$. That is, $x_{j}$ is fixed while $\mathbf{h}_{j}$ changes in SSK, whereas $x_{j}$ changes while $\mathbf{h}_{j}$ is fixed in APM. Therefore, decisions for APM are performed in the 1 - dim complex space, independent of which antenna is used (since after matched filtering, the sufficient statistics are scalar). On the other hand, SSK decisions are made in the $N_{\mathrm{r}}$ - dim space. We therefore expect SSK to outperform APM schemes for increasing $M$ and $N_{\mathrm{r}}$. Also, better performance is achieved in SSK for channel realizations having effective columns that are widely spread apart in the $N_{\mathrm{r}}$ - dim space, which depend on the stochastic properties of the channel. This spreading of columns is realized through adaptive forms of SSK (Section 4.3.1).

We note that, it is using the column indices of $\mathbf{H}$ as the source of information that results in the improved constellation space, and not the fact that different columns are being used for transmission. For example, if APM with transmission on alternating antenna indices is considered, $\mathcal{X}^{\text {eff }}$ would not change since, at any given time, 

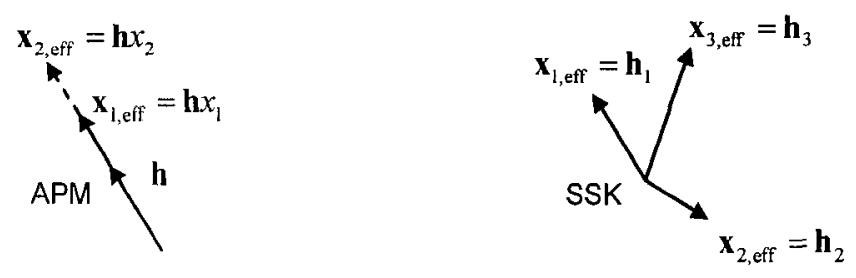

Figure 4.2: Illustration of the effective constellation space $\mathcal{X}^{\text {eff }}$.

the receiver explores only all possible transmit, symbols. The actual antenna index is assumed to be known at the receiver, and remains the same regardless of the transmitted symbol. If, on the other hand, both the antenna index and the transmit, symbol convey information, the modulation scheme is no longer APM, but rather SM. In this case, $\mathcal{X}^{\text {eff }}$ is similar to SSK, but with the possibility of having more than one scaled version of $\mathbf{x}_{j \text {,eff }}$ along the same direction (since both $x_{j}$ and $\mathbf{h}_{j}$ are changing).

\subsection{Performance Analysis}

We now investigate the analytical performance of the proposed SSK scheme. A tight upper bound on the bit error probability is derived, and we explicitly obtain SSK's diversity order. 


\subsubsection{Error Probability}

SSK's performance is derived similar to Section 3.2. The average BER for SSK is union bounded as

$$
\begin{aligned}
P_{\mathrm{e}, \mathrm{bit}}^{\mathrm{SSK}} & \leq E_{\mathbf{x}}\left[\sum_{\hat{\jmath} \neq j} N_{\mathrm{SSK}}(j, \hat{\jmath}) P_{\mathrm{SSK}}\left(\mathbf{x}_{j} \rightarrow \mathbf{x}_{\hat{\jmath}}\right)\right] \\
& =\sum_{j=1}^{N_{\mathrm{t}}} \sum_{\hat{\jmath}=j+1}^{N_{\mathrm{t}}} \frac{2 N_{\mathrm{SSK}}(j, \hat{\jmath})}{N_{\mathrm{t}}} P_{\mathrm{SSK}}\left(\mathbf{x}_{j} \rightarrow \mathbf{x}_{\hat{j}}\right),
\end{aligned}
$$

where $N_{\mathrm{SSK}}(j, \hat{\jmath})$ is the number of bits in error between the constellation vector $\mathbf{x}_{j}$ and $\mathbf{x}_{\hat{j}}$, and $P_{\mathrm{SSK}}\left(\mathbf{x}_{j} \rightarrow \mathbf{x}_{\hat{j}}\right)$ denotes the PEP of deciding on $\mathbf{x}_{\hat{j}}$ given that $\mathbf{x}_{j}$ is transmitted. Using (4.2), we obtain the PEP conditioned on $\mathrm{H}$ as

$$
P_{\mathrm{SSK}}\left(\mathbf{x}_{j} \rightarrow \mathbf{x}_{\hat{\jmath}} \mid \mathbf{H}\right)=P_{\mathrm{SSK}}\left(d_{\hat{\jmath}}>d_{j} \mid \mathbf{H}\right)=Q\left(\sqrt{\kappa_{\mathrm{SSK}}}\right)
$$

where $d_{j}=\operatorname{Re}\left\{\left(\mathbf{y}-\frac{\sqrt{\rho}}{2} \mathbf{h}_{j}\right)^{H} \mathbf{h}_{j}\right\}, Q(x)$ is defined by (3.3), and

$$
\kappa_{\mathrm{SSK}} \triangleq \frac{\rho}{2}\left\|\mathbf{h}_{j}-\mathbf{h}_{\hat{\jmath}}\right\|_{\mathrm{F}}^{2}=\sum_{n=1}^{2 N_{\mathrm{r}}} \alpha_{\mathrm{SSK}}^{2}(n),
$$

where $\alpha_{\mathrm{SSK}}(n) \sim \mathcal{N}\left(0, \sigma_{\mathrm{SSK}}^{2}\right)$ with $\sigma_{\mathrm{SSK}}^{2}=\frac{\rho}{2}$.

Remark 2 The metric affecting the system performance is the distance between the columns of $\mathbf{H}$. We hinted at this observation earlier in Section 4.1.3, when analyzing SSK's constellation. 
The random variable $\kappa_{\mathrm{SSK}}$ in (4.6) is chi-squared distributed with $s=2 N_{\mathrm{r}}$ degrees of freedom, and PDF given by (3.5) with $\sigma^{2}=\sigma_{\mathrm{SSK}}^{2}$. Similar to (3.6), the PEP can be formulated as

$$
P_{\mathrm{SSK}}\left(\mathbf{x}_{j} \rightarrow \mathbf{x}_{\hat{\jmath}}\right)=\gamma_{\mathrm{SSK}}^{N_{\mathrm{r}}} \sum_{k=0}^{N_{\mathrm{r}}-1}\left(\begin{array}{c}
N_{\mathrm{r}}-1+k \\
k
\end{array}\right)\left[1-\gamma_{\mathrm{SSK}}\right]^{k}
$$

where $\gamma_{\mathrm{SSK}}=\frac{1}{2}\left(1-\sqrt{\frac{\sigma_{\mathrm{SSK}}^{2}}{1+\sigma_{\mathrm{SSK}}^{2}}}\right)$. Plugging (4.7) into (4.5), we obtain

$$
P_{\mathrm{e}, \mathrm{bit}}^{\mathrm{SSK}} \leq \frac{N_{\Sigma} \gamma_{\mathrm{SSK}}^{N_{\mathrm{r}}}}{N_{\mathrm{t}}} \sum_{k=0}^{N_{\mathrm{r}}-1}\left(\begin{array}{c}
N_{\mathrm{r}}-1+k \\
k
\end{array}\right)\left[1-\gamma_{\mathrm{SSK}}\right]^{k},
$$

where $N_{\Sigma}=\sum_{j=1}^{N_{\mathrm{t}}} \sum_{\hat{\jmath}=j+1}^{N_{\mathrm{t}}} 2 N_{\mathrm{SSK}}(j, \hat{\jmath})$.

As we will see in Section 4.6.1, the bound in (4.8) is somewhat loose ( $\sim 1 \mathrm{~dB}$ offset for $M=8$ ), but captures the diversity of the system. The bound can be tightened further by considering only the number of nearest neighbors, $n_{\text {neigh }}$, in SSK's effective constellation space $\mathcal{X}^{\text {eff }}$, and is given by

$$
P_{\mathrm{e}, \mathrm{bit}}^{\mathrm{SSK}} \leq n_{\text {neigh }} N_{\mathrm{avg}} \gamma_{\alpha}^{N_{\mathrm{r}}} \sum_{k=0}^{N_{\mathrm{r}}-1}\left(\begin{array}{c}
N_{\mathrm{r}}-1+k \\
k
\end{array}\right)\left[1-\gamma_{\alpha}\right]^{k},
$$

where $N_{\text {avg }}=\frac{N_{\Sigma}}{N_{\mathrm{t}}\left(N_{\mathrm{t}}-1\right)}$ represents the average $N_{\mathrm{SSK}}(j, \hat{\jmath})$.

Remark 3 Since the effective constellation points $\mathbf{x}_{j, \text { eff }}$ are random, $n_{n e i g h}$ is a random variable. Intuitively, only one neighbor will exist since the probability of more 
than one $\mathbf{x}_{j, e f f}$ at the same distance from a particular $\mathbf{x}_{\hat{\jmath}, \text { eff }}$ is zero. Due to the nonsymmetric distribution of $\mathbf{x}_{j, \text { eff' }}$ 's, it is rather the neighbors around the neighborhood of $\mathbf{x}_{j, e f f}$ that affect performance. Also, it is clear that $n_{n e i g h}$ depends on $M$. For example, we will see from Section 4.6 .1 that for $M=8, n_{n e i g h} \approx 2$.

\subsubsection{Diversity}

The expression in (4.8) does not explicitly indicate the diversity achieved by SSK. In order to clearly show the system diversity, we re-derive the error probability with a loose upper bound. Specifically, we use $Q(x) \leq \frac{1}{2} \exp \left(-\frac{x^{2}}{2}\right)[28$, p. 54], and upper bound $P_{\mathrm{SSK}}\left(\mathbf{x}_{j} \rightarrow \mathbf{x}_{\hat{\jmath}}\right)=\int_{v=0}^{\infty} Q(\sqrt{v}) p_{\kappa}(v) d v$ by

$$
\begin{aligned}
P_{\mathrm{SSK}}\left(\mathbf{x}_{j} \rightarrow \mathbf{x}_{\hat{\jmath}}\right) & \leq \int_{0}^{\infty} \frac{\exp \left(-\frac{v}{2}\right) v^{N_{\mathrm{r}}-1} \exp \left(-\frac{v}{\rho}\right)}{2 \rho^{N_{\mathrm{r}}} \Gamma\left(N_{\mathrm{r}}\right)} d v \\
& =\frac{1}{2}\left(\frac{2}{\rho+2}\right)^{N_{\mathrm{r}}} \\
& \leq 2^{N_{\mathrm{r}}-1} \rho^{-N_{\mathrm{r}}}
\end{aligned}
$$

Therefore, the bit error probability is given by

$$
P_{\mathrm{e}, \mathrm{bjit}}^{\mathrm{SSK}} \leq C \rho^{-N_{r}}
$$

where $C=\frac{N_{\mathrm{SSK}}(j, \hat{\jmath}) 2^{N_{\mathrm{r}}-1}}{N_{\mathrm{t}}}$. We see from (4.10) that a diversity order of $N_{\mathrm{r}}$ is achieved, which is identical to an APM-MRC system using $N_{\mathbf{r}}$ receive antennas. 


\subsection{Adaptive SSK Transmission}

In this section, we consider systems with some CSI available at the transmitter. Two forms of CSI are considered: perfect channel knowledge at the transmitter, and limited feedback from the receiver. We present two schemes to exploit the available CSI, namely adaptive symbol design and transmit, AS.

\subsubsection{Adaptive Symbol Design}

In SSK, $x_{j}$ provides a degree of freedom in design to improve performance. Considering the ideal case when full transmit CSI is available, we exploit our PEP analysis (Section 4.2) to derive a mechanism for symbol design.

It is easy to show that the PEP for general power constrained symbols, conditioned on $\mathbf{H}$, is given by

$$
P_{\mathrm{SSK}}\left(\mathbf{x}_{j} \rightarrow \mathbf{x}_{\hat{\jmath}} \mid \mathbf{H}\right)=Q\left(\sqrt{\frac{\rho}{2}\left\|\mathbf{x}_{j, \mathrm{eff}}-\mathbf{x}_{\hat{j}, \mathrm{eff}}\right\|_{\mathrm{F}}^{2}}\right)
$$

where $\mathbf{x}_{j \text {,eff }}$ is defined in (4.4). The problem of minimizing the PEP for any constrained inputs reduces to the problem of finding $\mathcal{X}=\left\{x_{1}, x_{2}, \ldots, x_{M}\right\}, x_{j} \in \mathbb{C}$ such that $\mathbf{x}_{j \text {,eff' }}$ 's are maximally separated in $\mathbb{C}^{N_{\mathrm{r}}}$ (the $N_{\mathrm{r}}$ - dim complex vector space).

To simplify the optimization, we first consider the case when $N_{\mathbf{r}}=1$. One method to maximally separate $x_{j \text {,eff }}$ is as follows (we no longer use bold notation since for $N_{\mathrm{r}}=1$, we have complex scalars). If we choose $\left|x_{j}\right|=1$ for all $j$ (in order to satisfy power constraints mentioned in Section 4.1), we may express $x_{j, \text { eff }}=\left|h_{j}\right| e^{i\left(\theta_{h_{j}}+\theta_{x_{j}}\right)}$, 
where $i=\sqrt{-1}, x_{j}=\left|x_{j}\right| e^{i \theta_{x_{j}}}$, and $h_{j}=\left|h_{j}\right| e^{i \theta_{h_{j}}}$. Therefore, by designing $\theta_{x_{j}}$, we may place $x_{j, \text { eff }}$ around the complex plane with maximum distance from one another, with the design rule given by

$$
\theta_{x_{j}}=\frac{2 \pi j}{M}-\theta_{h_{j}}
$$

Another more complex, but more optimal design is to optimize both $\left|x_{j}\right|$ and $\theta_{x_{j}}$ simultaneously, keeping in mind power constraints.

For higher dimensions, the problem of symbol design is not straightforward. One simple, sub-optimal algorithm for $N_{\mathrm{r}}>1$ is to maximize the separation of the dimension with the most correlation, out of the $N_{\mathrm{r}}$ dimensions. The dimension with the most correlation, $\hat{k}$, is calculated by

$$
\hat{k}=\underset{k}{\arg \min } \sum_{j=1}^{M} \theta_{h_{k j}},
$$

where $\theta_{h_{k j}}$ is the angle of $h_{k j}$ (the element of $\mathbf{H}$ in the $k^{\text {th }}$ row, and $j^{\text {th }}$ column). Similar to the case of $N_{\mathrm{r}}=1$, the $\hat{k}^{\text {th }}$ dimension is then maximally separated using (4.11).

\subsubsection{Antenna Selection}

Another way to improve SSK's performance is through AS. We adopt a limited feedback based AS scheme, similar to that of [12], where the selection is based on minimizing the error rate. In particular, out of the available $N_{\mathrm{t}}$ antennas, we select $N_{\mathrm{s}}=M$ 
antennas for transmission. From the discussion about SSK's constellation (Section 4.1.3), it is clear that SSK's performance is improved by having more widely separated constellation points, which is achieved by selecting a subset from $N_{\mathrm{t}}$ antennas.

The subset of $N_{\mathrm{s}}$ antennas is obtained by minimizing the conditional symbol error probability, $P_{\mathrm{e}, \mathrm{symb} \mid \mathbf{H}}$, given by

$$
P_{\mathrm{e}, \mathrm{symb} \mid \mathbf{H}}^{\mathrm{SSK}} \leq \sum_{j=1}^{N_{\mathrm{t}}} \sum_{\hat{\jmath}=1, \hat{\jmath} \neq j}^{N_{\mathrm{t}}} Q\left(\sqrt{\frac{\rho}{2}}\left\|\mathbf{h}_{j} x_{j}-\mathbf{h}_{\hat{\jmath}} x_{\hat{\jmath}}\right\|_{\mathrm{F}}^{2}\right)
$$

Hence, the selection criterion is given by

$$
\hat{\mathbf{N}}_{\mathrm{t}}=\underset{\mathcal{N}_{\mathrm{t}}}{\arg \min } \sum_{j=1}^{N_{\mathrm{t}}} \sum_{\hat{\jmath}=j+1}^{N_{\mathrm{t}}} Q\left(\sqrt{\frac{\rho}{2}}\left\|\mathbf{h}_{j} x_{j}-\mathbf{h}_{\hat{\jmath}} x_{\hat{\jmath}}\right\|_{\mathrm{F}}^{2}\right),
$$

where $\hat{\mathbf{N}}_{\mathrm{t}}$ represents the set of selected antennas, and $\mathcal{N}_{\mathrm{t}}$ represents the $\left(\begin{array}{l}N_{\mathrm{t}} \\ N_{\mathrm{s}}\end{array}\right)$ possible antenna combinations. The optimization is performed at the receiver, and the selected index from $\mathcal{N}_{\mathrm{t}}$ (i.e., the index of $\mathcal{N}_{\mathrm{t}}$ containing $\hat{\mathbf{N}}_{\mathrm{t}}$ ) is fed back to the transmitter using $\log _{2}\left(\left(\begin{array}{l}N_{\mathrm{t}} \\ N_{\mathrm{s}}\end{array}\right)\right)$ bits.

\subsection{Generalized SSK}

As apparent from previous sections, the number of transmit antennas can grow quite large for higher SSK modulation order. We circumvent these difficulties by introducing a more flexible form of SSK, which we entitle as GSSK [30,31]. 


\subsubsection{Transmission and Detection}

In GSSK, $n_{\mathrm{t}}$ antennas remain active during transmission, and hence, $n_{\mathrm{t}}$ of the $x_{j}$ 's in $\mathbf{x}$ are nonzero. Therefore, there are $M^{\prime}=\left(\begin{array}{l}N_{\mathrm{t}} \\ n_{\mathrm{t}}\end{array}\right)$ possible constellation points. For example, with $n_{\mathrm{t}}=2$ and $N_{\mathrm{t}}=7$, there are $M^{\prime}=21$ possible combinations. Since we require a constellation size $M$ in multiples of 2 , we only use 16 of the possible 21 combinations. In order to meet hardware/complexity requirements for a fixed $M$, we can increase $n_{\mathrm{t}}$ while decreasing $N_{\mathrm{t}}$. The set of $M$ antenna combinations, $\mathcal{X}$, may be chosen at random, but more optimal selection rules exists (Section 4.4.3)

Once $\mathcal{X}$ is formulated, GSSK's mapper rule is straightforward. Groups of $m=$ $\log _{2}(M)$ bits are collected and mapped to a vector $\mathbf{x}_{\mathbf{j}}$, where $\mathbf{j} \in \mathcal{X}$ specifies the antenna combination for the given $m$ bit pattern. The symbols in $\mathbf{x}_{\mathbf{j}}$ do not contain information, but might also be designed to optimize transmission, similar to SSK's symbol design (Section 4.3.1). The vector $\mathbf{x}_{\mathbf{j}}$ specifies the activate antennas, during which all other antennas remain idle, and has the following form:

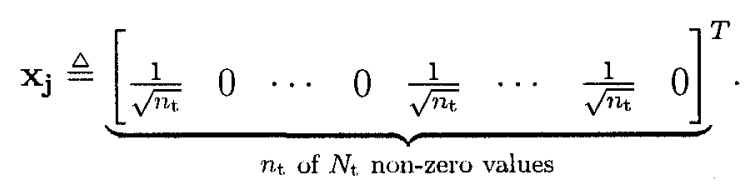

An example of 8-ary GSSK modulation is given in Table 4.2, where we use $N_{\mathrm{t}}=5$, $n_{\mathrm{t}}=2$, and $\mathcal{X}$ is chosen randomly. The output of the channel is therefore given by

$$
\mathbf{y}=\sqrt{\rho^{\prime}} \mathbf{h}_{\mathbf{j}, \text { eff }}+\boldsymbol{\eta}
$$


Table 4.2: Example of the GSSK Mapper Rule.

\begin{tabular}{|c|c|c|}
\hline $\mathbf{b}=\left[\begin{array}{lll}b_{1} & b_{2} & b_{3}\end{array}\right]$ & $\mathbf{j}$ & $\mathbf{x}=\left[\begin{array}{llll}x_{1} & x_{2} & \cdots & x_{5}\end{array}\right.$ \\
\hline$\left[\begin{array}{lll}0 & 0 & 0\end{array}\right]$ & $(1,2)$ & $\left.\begin{array}{lllll}\frac{1}{\sqrt{2}} & \frac{1}{\sqrt{2}} & 0 & 0 & 0\end{array}\right]$ \\
\hline$\left[\begin{array}{lll}0 & 0 & 1\end{array}\right]$ & $(1,3)$ & $\left.\begin{array}{lllll}\frac{1}{\sqrt{2}} & 0 & \frac{1}{\sqrt{2}} & 0 & 0\end{array}\right]$ \\
\hline$\left[\begin{array}{lll}0 & 1 & 0\end{array}\right]$ & $(1,4)$ & $\left.\begin{array}{lllll}\frac{1}{\sqrt{2}} & 0 & 0 & \frac{1}{\sqrt{2}} & 0\end{array}\right]$ \\
\hline$\left[\begin{array}{lll}0 & 1 & 1\end{array}\right]$ & $(1,5)$ & $\left.\frac{1}{\sqrt{2}} \quad 0 \quad 0 \quad 0 \quad 00 \quad \frac{1}{\sqrt{2}}\right]$ \\
\hline$\left[\begin{array}{lll}1 & 0 & 0\end{array}\right]$ & $(2,3)$ & {$\left[\begin{array}{lllll}0 & \frac{1}{\sqrt{2}} & \frac{1}{\sqrt{2}} & 0 & 0\end{array}\right]$} \\
\hline$\left[\begin{array}{lll}1 & 0 & 1\end{array}\right]$ & $(2,4)$ & $\left.\begin{array}{lllll}0 & \frac{1}{\sqrt{2}} & 0 & \frac{1}{\sqrt{2}} & 0\end{array}\right]$ \\
\hline$\left[\begin{array}{lll}1 & 1 & 0\end{array}\right]$ & $(2,5)$ & {$\left[\begin{array}{lllll}0 & \frac{1}{\sqrt{2}} & 0 & 0 & \frac{1}{\sqrt{2}}\end{array}\right]$} \\
\hline$\left[\begin{array}{lll}1 & 1 & 1\end{array}\right]$ & $(3,4)$ & {$\left[\begin{array}{lllll}0 & 0 & \frac{1}{\sqrt{2}} & \frac{1}{\sqrt{2}} & 0\end{array}\right]$} \\
\hline
\end{tabular}

where $\rho^{\prime}=\frac{\rho}{n_{\mathrm{t}}}$, and $\mathbf{h}_{\mathbf{j}, \text { eff }}=\mathbf{h}_{\mathbf{j}(1)}+\mathbf{h}_{\mathbf{j}(2)}+\cdots+\mathbf{h}_{\mathbf{j}\left(n_{\mathrm{t}}\right)}\left(\mathbf{j}(\cdot) \doteq j \in\left\{1,2, \ldots, N_{\mathrm{t}}\right\}\right.$ specifies the column index of $\mathbf{H}$ ). We refer to $\mathbf{h}_{\mathbf{j}, \text { eff }}$ as an effective column, which represents the sum of $n_{\mathrm{t}}$ distinct columns in $\mathbf{H}$.

Remark 4 Only $n_{t}$ columns of $\mathbf{H}$ are activated, and these columns change depending on the transmitted information. Essentially, these effective columns act as random constellation points for GSSK modulation.

Since the channel inputs are assumed equally likely, the optimal detector is ML, which is given by

$$
\mathbf{k}=\underset{\mathbf{j}}{\arg \max } \operatorname{Re}\left\{\left(\mathbf{y}-\frac{\sqrt{\rho^{\prime}}}{2} \mathbf{h}_{\mathbf{j}, \mathrm{eff}}\right)^{H} \mathbf{h}_{\mathbf{j}, \text { eff }}\right\}
$$

where $\mathbf{k} \in \mathcal{X}$ represents the estimated antenna indices. The detection rule is hence a maximization problem over all effective columns of $\mathbf{H}$ (there are $M$ of them). 


\subsubsection{Performance Analysis}

GSSK's performance is easily obtained from the analysis in Section 4.2.1, by replacing

$j \rightarrow \mathbf{j}, \hat{\jmath} \rightarrow \mathbf{k}, \mathbf{h}_{j} \rightarrow \mathbf{h}_{\mathbf{j}, \text { eff }}, \mathbf{h}_{\hat{\jmath}} \rightarrow \mathbf{h}_{\mathbf{k}, \mathrm{eff}}, \rho \rightarrow \rho^{\prime}, \kappa_{\mathrm{SSK}} \rightarrow \kappa_{\mathrm{GSSK}}=\frac{\rho^{\prime}}{2}\left\|\mathbf{h}_{\mathbf{j}, \mathrm{eff}}-\mathbf{h}_{\mathbf{k}, \mathrm{eff}}\right\|_{\mathbf{F}}^{\mathbf{2}}$, and $\sigma_{\mathrm{SSK}}^{2} \rightarrow \sigma_{\mathrm{GSSK}}^{2}=\frac{\rho^{\prime} d(\mathbf{j}, \mathbf{k})}{4}$, where $d(\mathbf{j}, \mathbf{k})$ is the number of distinct columns of $\mathbf{H}$ between $\mathbf{h}_{\mathbf{j}, \text { eff }}$ and $\mathbf{h}_{\mathbf{k}, \mathrm{eff}}$. We then obtain

$$
P_{\mathrm{e}, \mathrm{bit}}^{\mathrm{GSSK}} \leq \frac{1}{M} \sum_{\mathbf{j}} \sum_{\mathbf{k}} N_{\mathrm{GSSK}}(\mathbf{j}, \mathbf{k}) \gamma_{\mathrm{GSSK}}^{N_{\mathrm{r}}} \sum_{k=0}^{N_{\mathrm{r}}-1}\left(\begin{array}{c}
N_{\mathrm{r}}-1+k \\
k
\end{array}\right)\left[1-\gamma_{\mathrm{GSSK}}\right]^{k}
$$

where $\gamma_{\mathrm{GSSK}}=\frac{1}{2}\left(1-\sqrt{\frac{\sigma_{\mathrm{GSSK}}^{2}}{1+\sigma_{\mathrm{GSSK}}^{2}}}\right)$ and $N_{\mathrm{GSSK}}(\mathbf{j}, \mathbf{k})$ is the number of bits in error between the constellation vector $\mathbf{x}_{\mathbf{j}}$ and $\mathbf{x}_{\mathbf{k}}$.

Remark 5 Note that, with $N_{t}=M$ and $n_{t}=1$, we get $d(\mathbf{j}, \mathbf{k})=2$, and (4.16) reduces to SSK's error probability expression of (4.8).

Also, similar to Section 4.2.2, the diversity of GSSK is easily obtained to be $N_{\mathrm{r}}$ by upper bounding the bit error probability as $P_{\mathrm{e}, \mathrm{bit}} \leq C \rho^{-N_{\mathbf{r}}}$ where

$$
C=\sum_{\mathbf{j}} \sum_{\mathbf{k}} \frac{N_{\mathrm{GSSK}}(\mathbf{j}, \mathbf{k})}{M} 2^{2 N_{\mathbf{r}}+\mathbf{1}}\left(\frac{d(\mathbf{j}, \mathbf{k})}{n_{\mathrm{t}}}\right)^{-N_{\mathbf{r}}}
$$




\subsubsection{Optimal Constellation Design}

We consider the optimal formulation of $\mathcal{X}$ in terms of minimizing the BER. The optimal constellation is therefore obtained by the following joint optimization problem:

$$
\hat{\Psi}_{\text {opt }}=\underset{\Psi_{\text {opt }}}{\arg \min } P_{\mathrm{e}, \mathrm{bit}}^{\mathrm{GSSK}}
$$

where $\hat{\Psi}_{\text {opt }}=\left(\hat{N}_{\mathrm{t}}, \hat{n}_{\mathrm{t}}, \hat{\mathcal{X}}, \hat{\mu}\right), \Psi_{\text {opt }}=\left(N_{\mathrm{t}}, n_{\mathrm{t}}, \mathcal{X}, \mu\right)$ with constraint $n_{\mathrm{t}}<N_{\mathrm{t}}$ and $M^{\prime} \geq M, \mu$ is the labeling rule for the constellation $\mathcal{X}$ (i.e., the rule for labeling $m$ bits to a symbol vector $\mathrm{x}_{\mathrm{j}}$ ), and $P_{\mathrm{e}, \mathrm{bit}}^{\mathrm{GSSK}}$ is given by (4.16). We note that in (4.16), $N_{\mathrm{GSSK}}(\mathbf{j}, \mathbf{k})$ is affected by $\mu$, whereas $\gamma_{\mathrm{GSSK}}$ is affected by $n_{\mathrm{t}}, N_{\mathrm{t}}$, and $\mathcal{X}$. To simplify the design process, we present two sub-optimal constellation formulations.

In the first algorithm, we simplify the optimization problem by considering relatively high SNRs. Hence, (4.17) reduces to

$$
\hat{\Psi}_{1}=\underset{\Psi_{1}}{\arg \min } \sum_{\mathbf{j}} \sum_{\mathbf{k}} N_{\mathrm{GSSK}}(\mathbf{j}, \mathbf{k}) \gamma_{\mathrm{GSSK}}
$$

where we only keep the terms contributing most to $P_{\mathrm{e}, \mathrm{bit}}^{\mathrm{GSSK}}$, and $\hat{\Psi}_{1}$ and $\Psi_{1}$ have the same parameters as $\hat{\Psi}_{\text {opt }}$ and $\Psi_{\text {opt }}$, respectively. Still, the optimization is fairly complex (remember, for each $N_{\mathrm{t}}$ chosen, there are $N_{\mathrm{t}}-1$ possibilities for $n_{\mathrm{t}}$, from

which there are $\left(\begin{array}{c}N_{\mathrm{t}} \\ n_{\mathrm{t}} \\ M\end{array}\right)$ available antenna combinations to formulate $\left.\mathcal{X}\right)$. Therefore, to simplify the optimization further, we consider only minimizing the parameter $\gamma_{\mathrm{GsSK}}$. 
The second constellation design rule is therefore given by

$$
\begin{aligned}
\hat{\Psi}_{2} & =\underset{\Psi_{2}}{\arg \max } \sum_{\mathbf{j}} \sum_{\mathbf{k}} \sigma_{\mathrm{GSSK}}^{2} \\
& =\underset{\Psi_{2}}{\arg \max } \frac{1}{n_{\mathrm{t}}} \sum_{\mathbf{j}} \sum_{\mathbf{k}} d(\mathbf{j}, \mathbf{k})
\end{aligned}
$$

where $\hat{\Psi}_{2}=\left(\hat{N}_{\mathrm{t}}, \hat{n}_{\mathrm{t}}, \hat{\mathcal{X}}\right), \Psi_{2}=\left(N_{\mathrm{t}}, n_{\mathrm{t}}, \mathcal{X}\right)$. For a given $N_{\mathrm{t}}$ and $n_{\mathrm{t}}$ value, this maximization can be interpreted as having antenna combinations that are as different from one another as possible. Widely varying antenna combinations imply having a large $M^{\prime}$, which is increased by choosing a larger $N_{\mathrm{t}}$, and thus increasing the transmitter's overhead. Note that a large $n_{\mathrm{t}}$ will also help, but will have adverse effects as well due to the $\frac{1}{n_{\mathrm{t}}}$ factor in (4.19). Depending on system requirements (i.e., low hardware overhead, or high performance), the range of requirements for $N_{\mathrm{t}}$ and $n_{\mathrm{t}}$ can be specified, from which the optimal combination of $N_{\mathrm{t}}$ and $n_{\mathrm{t}}$ can be obtained by (4.19) through computer search.

Remark 6 The optimal set of parameters may not be unique, since several sets may result in identical performance. Also, this trade-off between transmitter complexity and performance provides design flexibility, which can be further exploited in adaptive type systems.

Figure 4.3 illustrates GSSK's performance bounds given by (4.16), with $M=8$ and $M=32$, and for varying $N_{\mathrm{t}}$ 's. For each plot, $n_{\mathrm{t}}$ and $\mathcal{X}$ are obtained by computer simulations using (4.19). 


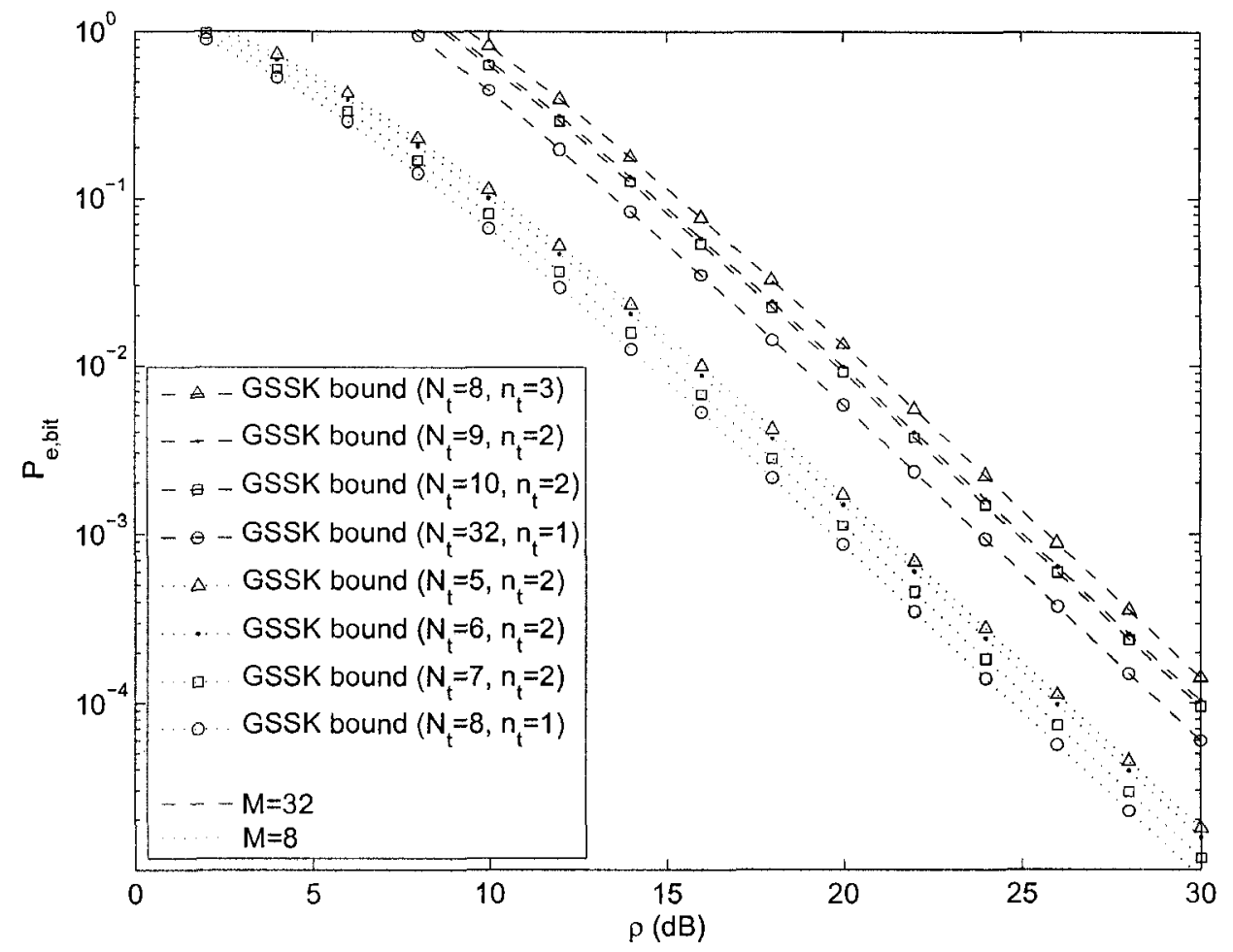

Figure 4.3: GSSK bounds for varying $M, N_{\mathrm{t}}$, and $n_{\mathrm{t}}\left(N_{\mathrm{r}}=2\right)$.

As expected, the performance degrades as $N_{\mathrm{t}}$ decreases, and as $n_{\mathrm{t}}$ increases. We also note that certain antenna transitions do not gain much in performance. For example, only a few tenths of a dB is gained from the transition of $N_{\mathrm{t}}=9$ to $N_{\mathrm{t}}=10$ $(M=32)$

\subsection{Complexity}

In this section, we compare SSK's complexity to that of SM-OD. Since SM-OD is shown to exhibit similar complexity to that of SM (Section 3.3), the reader is referred 
to [22] for other complexity comparisons (such as V-BLAST).

In our presentation, we quantify complexity by the number of multiplications required in the detection process. The number of additions can be shown to have a similar value for both detectors. In Section 3.3, the complexity of SM-OD is given by $\delta_{\mathrm{SM}-\mathrm{OD}}=2 N_{\mathrm{r}} N_{\mathrm{t}}+N_{\mathrm{t}} M+M$. Similar to [22], we analyze (4.2) to obtain SSK's complexity (also applicable to GSSK), which is given by

$$
\delta_{\mathrm{SSK}}=N_{\mathrm{r}} M
$$

It is important to note that, for the same spectral efficiency, the value of $N_{\mathrm{t}}$ and $M$ for SM-OD and SSK are different. For SM-OD, $M$ denotes the constellation size of the APM scheme, where the total constellation size of SM-OD is $M N_{\mathrm{t}}$. As well, we note that for GSSK, $N_{\mathrm{t}}$ and $n_{\mathrm{t}}$ are functions of $M$. At first glance, it is not straightforward to compare both detector complexities. However, for practical values of $N_{\mathrm{t}}, N_{\mathrm{r}}$, and $M$, SSK (and GSSK) can be shown to have lower complexity. For example, let us fix $m=3$ bits $/ \mathrm{s} / \mathrm{Hz}$ transmission $\left(N_{\mathrm{r}}=2\right)$, and use $N_{\mathrm{t}}=4$ and $M=2$ for SM-OD. We then obtain $\delta_{\mathrm{SSK}}=16$, and $\delta_{\mathrm{SM}-\mathrm{OD}}=26$. In this case, SM-OD requires more than $50 \%$ complex multiplications than SSK.

\subsection{Simulation Results}

In this section, we present some examples to compare SSK's performance for varying parameters (such as $M$ and $N_{\mathrm{r}}$ ). Monte Carlo simulations are performed, and are run 
for at least $10^{5}$ channel realizations. All results consider a Rayleigh fading channel, as described in Section 4.1, with complete channel knowledge at the receiver. We also use Gray (or quasi-Gray) mapping when appropriate (i.e., for PSK and QAM modulation). The plots illustrate the average BER performance versus $\rho$.

\subsubsection{GSSK versus SM and MRC}

In Fig. 4.4, we demonstrate SSK's performance versus MRC, and SM. We target $m=3 \mathrm{bits} / \mathrm{s} / \mathrm{Hz}$ transmission, with $N_{\mathrm{r}}=4$. For reference, two different transmission setups are used. The first one is APM, 8-QAM transmission with $N_{\mathrm{t}}=1$, and MRC. The second is SM-OD (Section 3.1) with $N_{\mathrm{t}}=4$ antennas, and BPSK modulation.

Since SSK is a special case of GSSK, we plot GSSK for various antenna configurations as well, where SSK is the case when $N_{\mathrm{t}}=M\left(n_{\mathrm{t}}=1\right)$. The first configuration is with $N_{\mathrm{t}}=M=8$, and hence, $n_{\mathrm{t}}=1$. The second is with $N_{\mathrm{t}}=5, n_{\mathrm{t}}=2$, and $\mathcal{X}$ is obtained from (4.19). The bounds of (4.8), (4.9), and (4.16) are also plotted for comparison. The simulation and analytical results are a close match, especially at high SNRs. The bounds are tighter for $n_{\mathrm{t}}=2$ than $n_{\mathrm{t}}=1$, and this is due to the fact that summing over all possible constellation points (for the union bound) is more justified in the case of $n_{\mathrm{t}}=2$. When $n_{\mathrm{t}}=1$, the constellation points are all unique (i.e., distinct columus of $\mathbf{H}$ ), resulting in a union bound that is more loose (in this case, the nearest neighbor approximation is better suited). On the other hand, with $n_{\mathrm{t}}=2$, the constellation points share common columns of $\mathbf{H}$, and a larger number of constellation points have an effect on performance. Therefore, the union bound for 


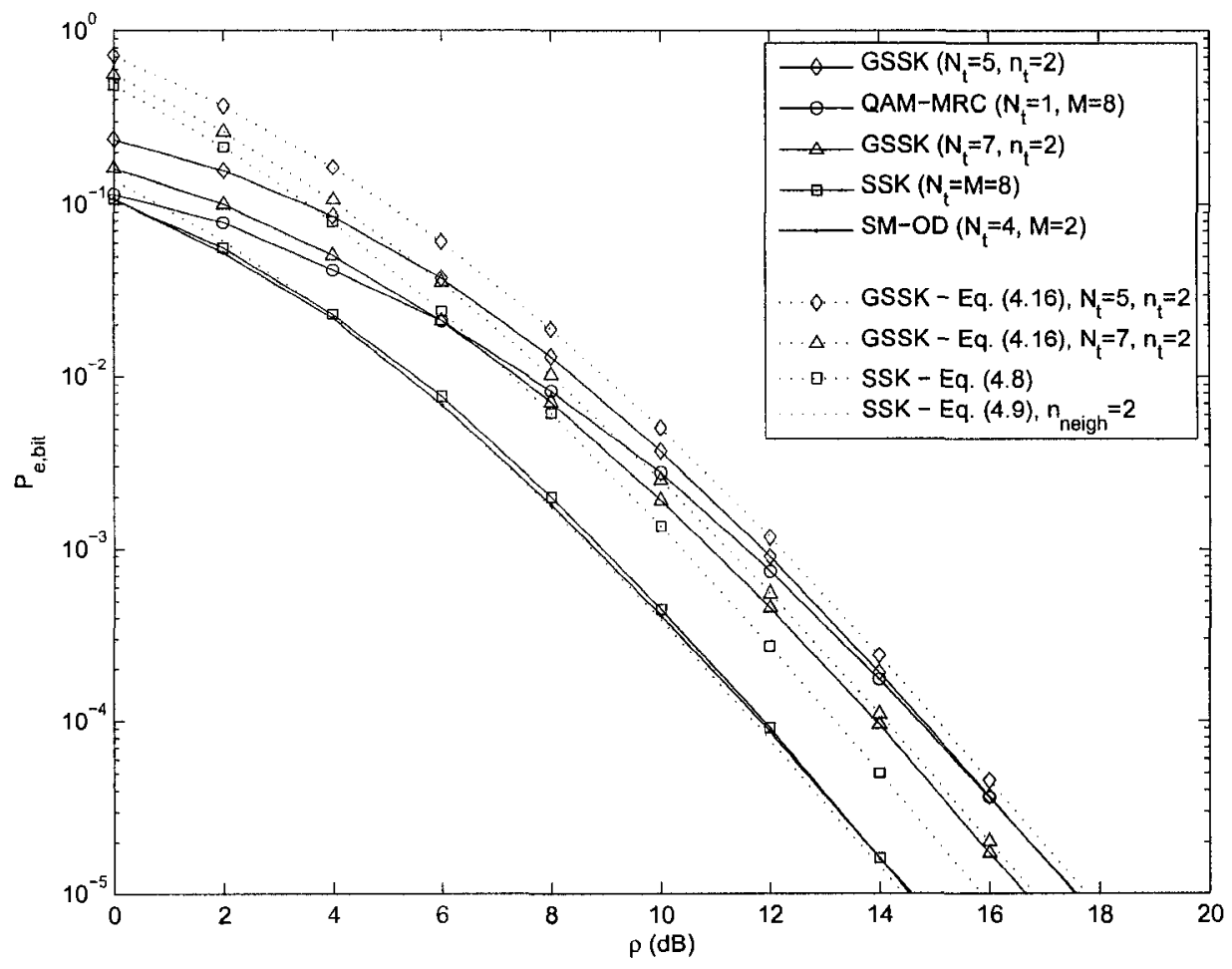

Figure 4.4: BER performance of GSSK versus MRC, and SM, for $m=3$ bits $/ \mathrm{s} / \mathrm{Hz}$ transmission $\left(N_{\mathrm{r}}=4\right)$.

these higher $n_{\mathrm{t}}$ values are more tight.

SSK's performance improvements is clearly shown in Fig. 4.4, where we observe gains of $3 \mathrm{~dB}$ over APM. SSK has almost identical performance to that of SM-OD, but with lower complexity (attributed to the fact that symbols do not carry information such as in SM and APM-MRC). We also note that, as expected, GSSK's performance degrades as $N_{\mathrm{t}}$ is decreased, but still outperforms APM in most cases. 


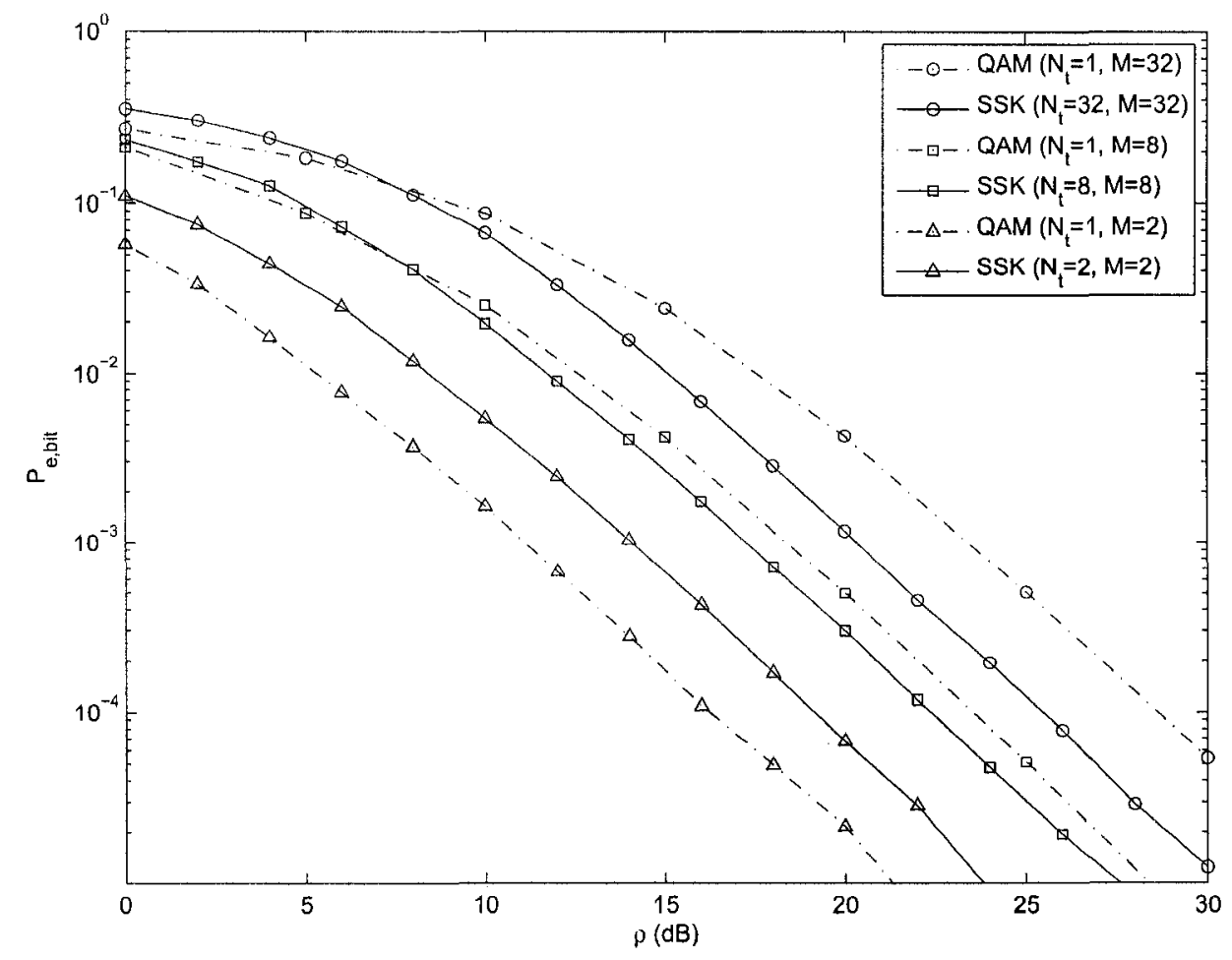

Figure 4.5: BER performance of SSK versus MRC, for varying $M\left(N_{\mathrm{r}}=2\right)$.

\subsubsection{SSK versus APM (varying $M$ )}

In this example, we illustrate the effect of the constellation size $M$ on performance.

Fig. 4.5 shows the BER performance of SSK and APM schemes for $M=2,8$, and

32. For $M \leq 4$, QAM outperforms SSK, and we expect this from the discussion in

Section 4.1.3. However, for $M=8$ onwards, SSK outperforms QAM, and the gap broadens for increasing $M$, where gains of up to $4 \mathrm{~dB}$ are observed at $P_{\text {e,bit }}=10^{-4}$ (for $M=32$ ). 


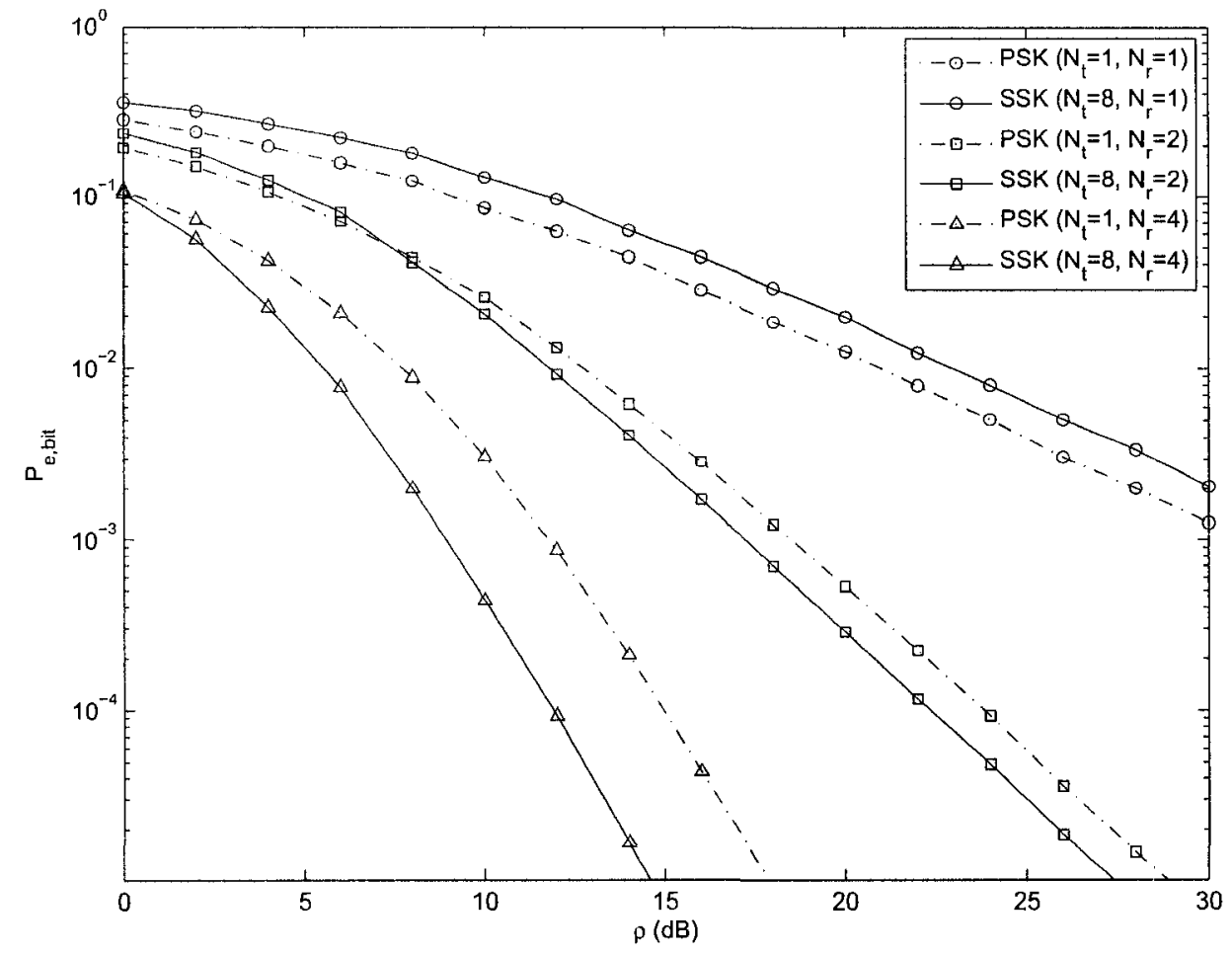

Figure 4.6: BER performance of SSK versus MRC, for varying $N_{\mathbf{r}}(M=8)$.

\subsubsection{SSK versus APM (varying $N_{\mathrm{r}}$ )}

We now investigate the effect of varying $N_{\mathrm{r}}$ on the performance of SSK. We target $m=3 \mathrm{bits} / \mathrm{s} / \mathrm{Hz}$, and hence consider 8-QAM and 8-SSK modulation. Figure 4.6 illustrates the average BER performance as $N_{\mathrm{r}}$ is varied. We see that SSK's diversity order is identical to APM. However, SSK achieves SNR gains of about $4 \mathrm{~dB}$ over APM at, $P_{\text {e,bit }}=10^{-5}\left(N_{\mathrm{r}}=4\right)$. Also, SSK gains more in terms of performance as $N_{\mathrm{r}}$ is varied compared to APM. For example, for $N_{\mathbf{r}}=2$ to $N_{\mathrm{r}}=4$, SSK gains $12 \mathrm{~dB}$ whereas PSK only gains about $10 \mathrm{~dB}$. 


\subsubsection{SSK Adaptive Symbol Design}

Figure 4.7 illustrates the performance improvements with adaptive symbol design using (4.11), with as much as $12 \mathrm{~dB}$ in gains at, $P_{\mathrm{e}, \mathrm{bit}}=10^{-3}$. These gains are analogous to the gains obtainable by optimal power allocation for APM schemes in fading channels, where the performance becomes closer to that of an unfaded channel [32]. The gains observed in Fig. 4.7 are not practical, since perfect channel phase is required at the transmitter. However, we still expect reasonable performance improvements when quantized, less accurate CSI is obtained in the form of limited receiver feedback [33].

Figure 4.8 illustrates the performance of the sub-optimal symbol design using (4.12), for $N_{\mathrm{r}}=2$, and 4. We notice smaller gains as $N_{\mathrm{r}}$ is increased, due to the suboptimality of the algorithm, which minimizes the correlation of the most correlated dimension without taking into account the change in correlation on the other dimensions. Therefore, although the weakest dimension becomes minimally correlated, another dimension may have opposite effects. This is more likely to occur when $N_{\mathrm{r}}$ is large, and is representative of the results in Fig. 4.8 .

\subsubsection{SSK Antenna Selection}

Figure 4.9 illustrates the performance for SSK-AS using (4.13) with $M=2$. Tremendous gains are observed, where only one extra antenna is available to choose from (i.e., $N_{\mathrm{t}}=N_{\mathrm{s}}+1$ ). As well, there seems to be an increase in diversity as the number 


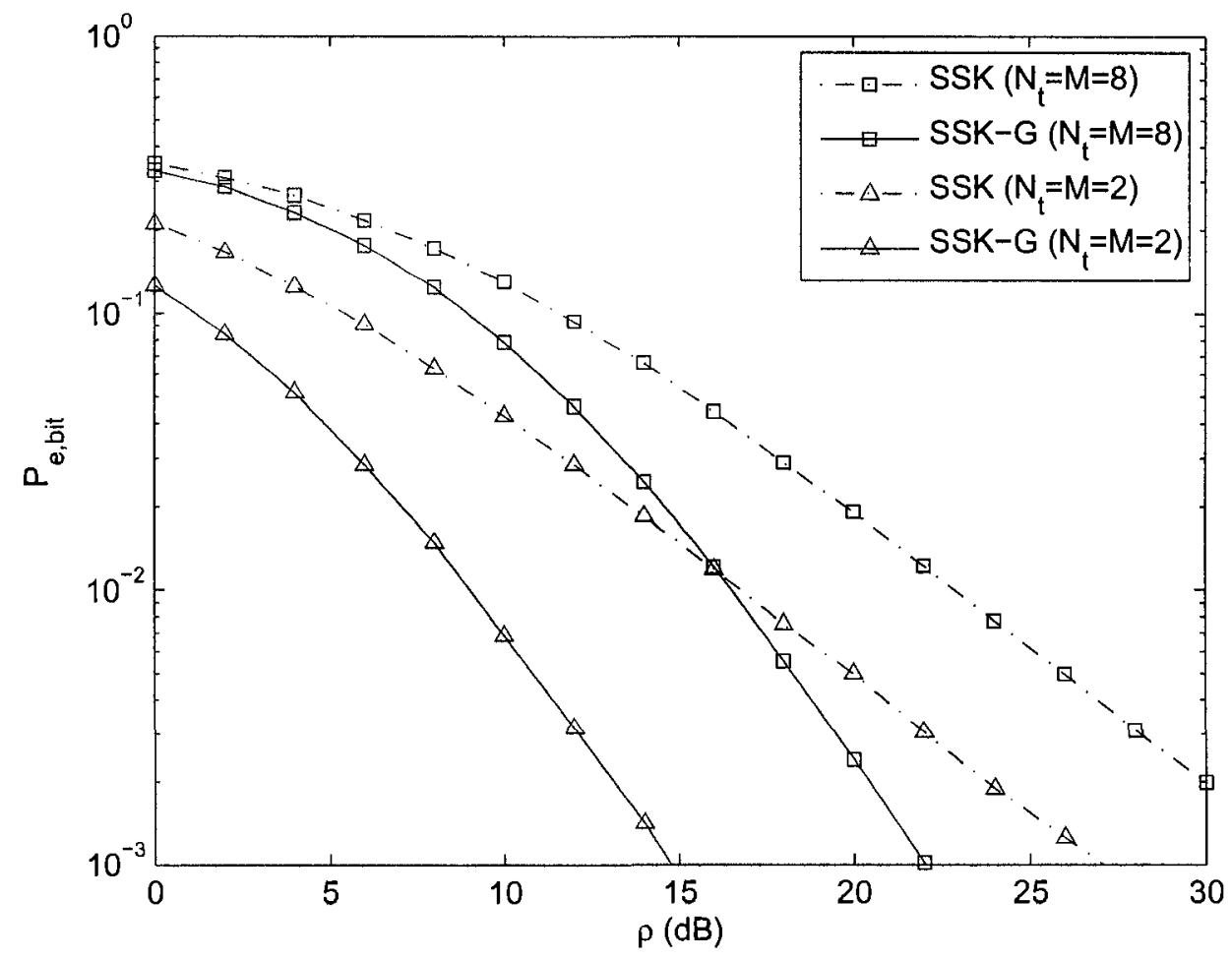

Figure 4.7: BER performance of SSK with adaptive symbol design, for varying $M$ $\left(N_{\mathrm{r}}=1\right)$.

of extra antennas to choose from is increased (i.e., as $N_{\mathrm{t}}-N_{\mathrm{s}}$ increases). This diversity increase is somewhat equivalent to transmit antenna selection, where a diversity order of $\left(N_{\mathrm{t}}-N_{\mathrm{s}}+1\right) N_{\mathrm{r}}$ is shown [13]. Additional diversity increase is possible by also varying $N_{\mathrm{s}}$, similar to [16]. We conjecture that in the case of such multimode SSK-AS, a diversity order of $N_{\mathrm{t}} N_{\mathrm{r}}$ is possible. 


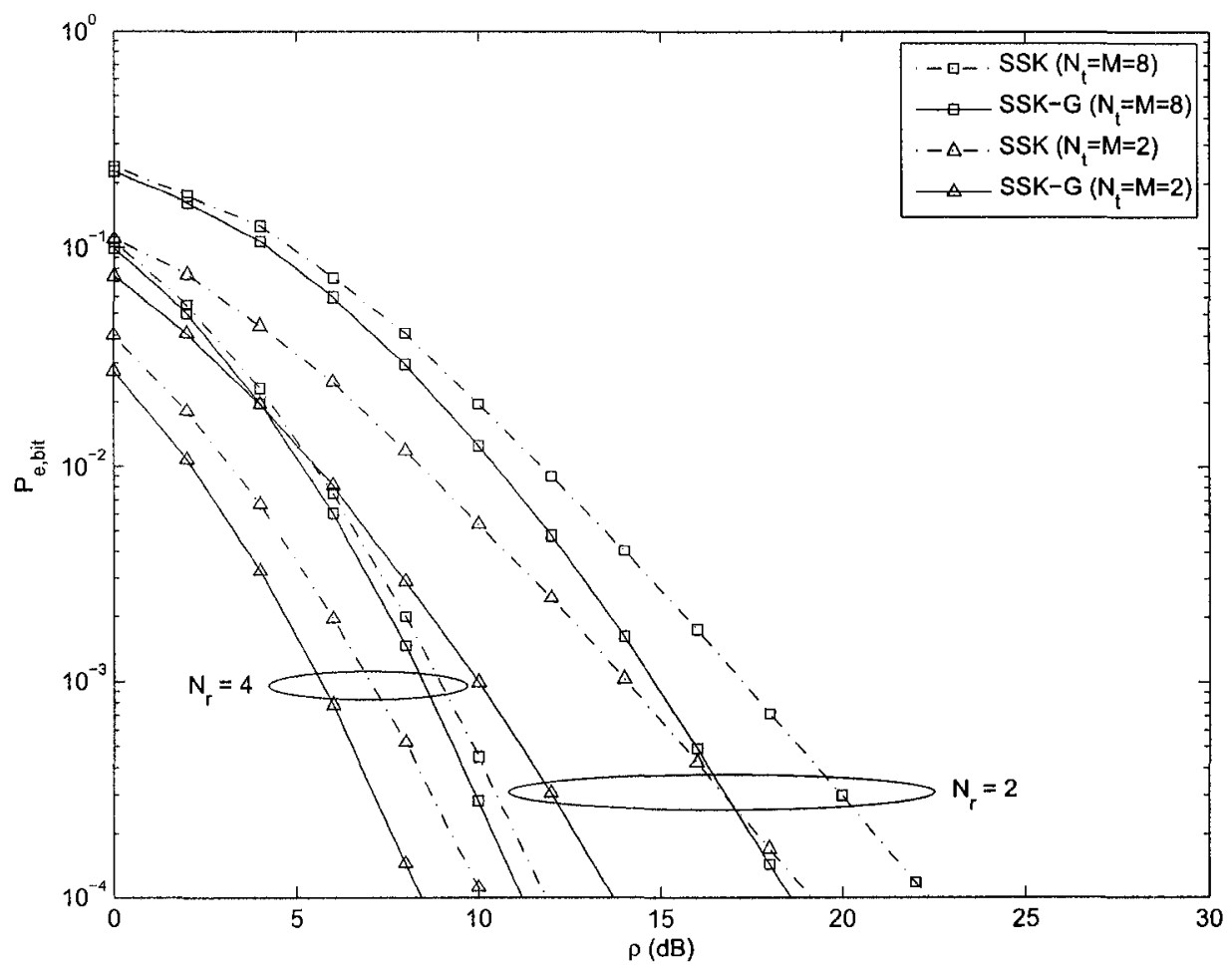

Figure 4.8: BER performance of SSK with sub-optimal adaptive symbol design, for varying $M$ and $N_{\mathrm{r}}$.

\subsubsection{SSK Under Non-ideal Conditions}

In this example, the effect of certain real-world non-idealities on the performance of SSK is studied. In particular, we present two scenarios. In the first, we consider CSI estimation effects on system performance, while in the second, spatial correlation (SC) amongst transmit and receive antennas is considered.

CSI estimation: Estimation of the channel matrix $\mathbf{H}$ is performed using pilot symbols along with the well known least square (LS) estimation method [34]. Other estimation techniques may also be considered [26, Ch. 10]. The estimated channel, 


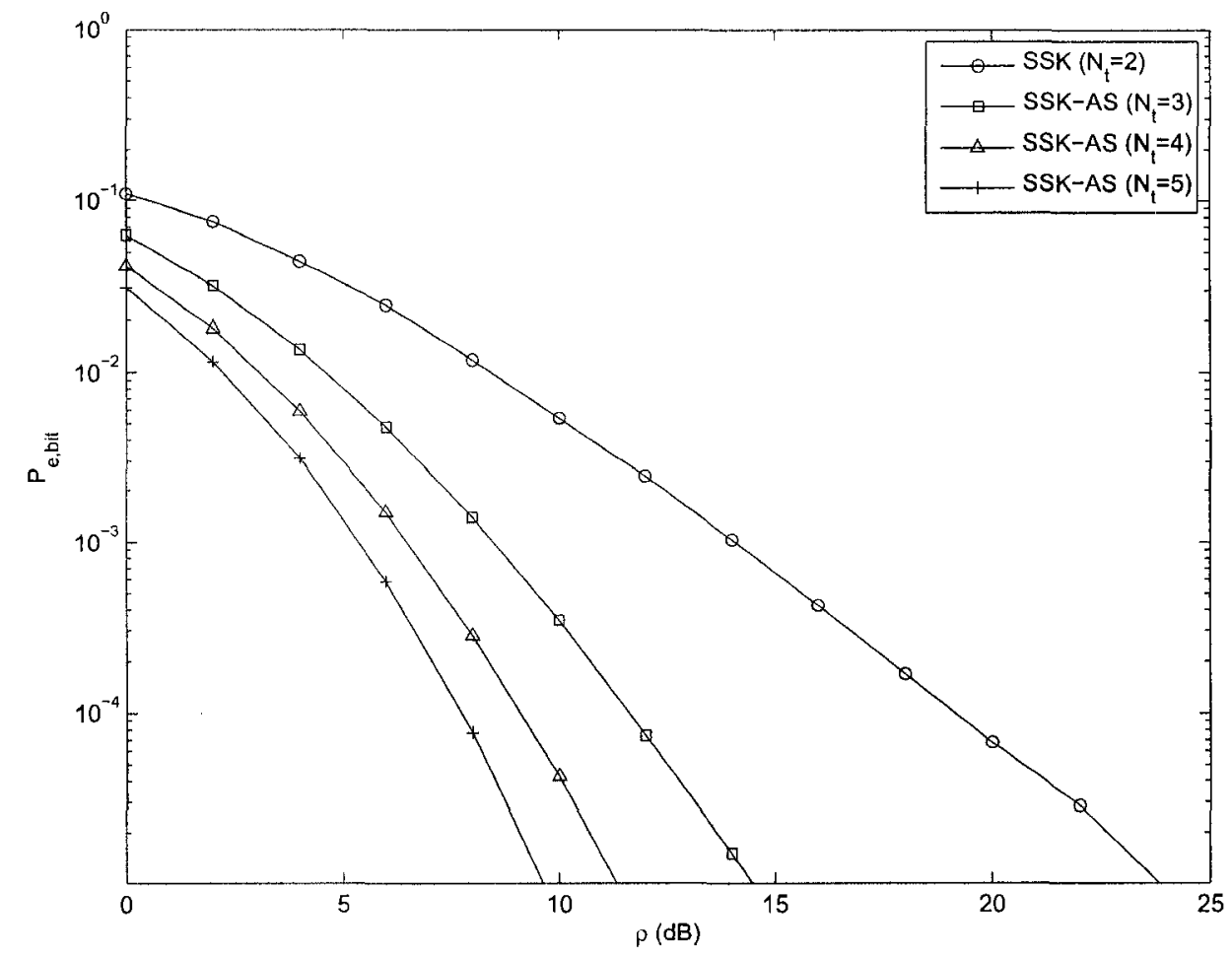

Figure 4.9: BER performance of SSK with antenna selection, for varying $N_{\mathrm{t}}\left(N_{\mathrm{s}}=\right.$ $M=2, N_{\mathrm{r}}=2$ ).

$\hat{\mathbf{H}}_{\mathrm{LS}}$, is given by $\hat{\mathbf{H}}_{\mathrm{LS}}=\frac{1}{\sqrt{\rho}} \mathbf{y}_{\mathrm{p}} \mathbf{x}_{\mathrm{p}}^{H}\left(\mathbf{x}_{\mathrm{p}} \mathbf{x}_{\mathrm{p}}^{H}\right)^{-1}$, where the subscript $\mathrm{p}$ is used to indicate that the input and output are obtained using pilot symbols. In [35], it is shown that $\mathbf{x}_{\mathrm{p}}$ using constant energy orthogonal rows make for optimal pilot symbols. Therefore, we use $\mathbf{x}_{\mathrm{p}}=\mathbf{I}_{N_{\mathrm{t}}}$, which is essentially a symbol set taken form SSK modulation. With such inputs, the LS solution becomes $\hat{\mathbf{H}}_{\mathrm{LS}}=\mathbf{H}+\frac{\eta}{\sqrt{\rho}}$. Therefore, the effect of estimation error is simply a shift in SSK's performance, attributed to the fact that the estimated channel is now distributed according to $\mathcal{C N}\left(0, \frac{1+\rho}{\rho}\right)$.

Spatial Correlation: Assuming the presence of local scatters around both transmitter and receiver, one widely accepted model for spatial correlation (SC) is given 
by $[36]$

$$
\mathbf{H}_{\text {curr }}=\mathbf{R}_{\mathbf{r}}^{\frac{1}{2}} \mathbf{H R}_{\mathrm{t}}^{\frac{1}{2}}
$$

where $\mathbf{R}^{\frac{1}{2}} \mathbf{R}^{\frac{1}{2}}=\mathbf{R}$. In our presentation of SC, we obtain the correlation matrices according to $[37]$

$$
\mathbf{R}(r, c)=\mathbf{R}^{*}(c, r)=\rho_{\text {corr }}^{r-c}, \quad r \geq c
$$

where $\mathbf{R}(r, c)$ represents the element of $\mathbf{R}$ in the $r^{\text {th }}$ row and $c^{\text {th }}$ column, and $\rho_{\text {corr }}$ represents the amount of correlation $\left(\left|\rho_{\text {corr }}\right|<1\right)$. Equation $(4.20)$ may not be an accurate method of generating the correlation coefficients in real world scenarios. However, this simple parameter model gives us quick insight into the effects of SC on SSK modulation. Note that the model does follow some reasonable physical characteristics, such as the decrease in correlation with the increase between antenna distance. More realistic correlation matrices can be obtained given specific channel scenarios [38,39], which vary depending on the application.

\section{(Non-ideal) SSK for Varying $M$}

Figure 4.10 illustrates SSK's performance under non-ideal scenarios, for varying modulation size $M$.

As expected, the degradation in performance due to CSI estimation error is relatively the same for both $M$ values (about $3 \mathrm{~dB})$. With SC $\left(\rho_{\text {corr }}=0.5\right)$ on the other hand, SSK experiences larger loss in performance for $M=2$ than $M=32$, which is 


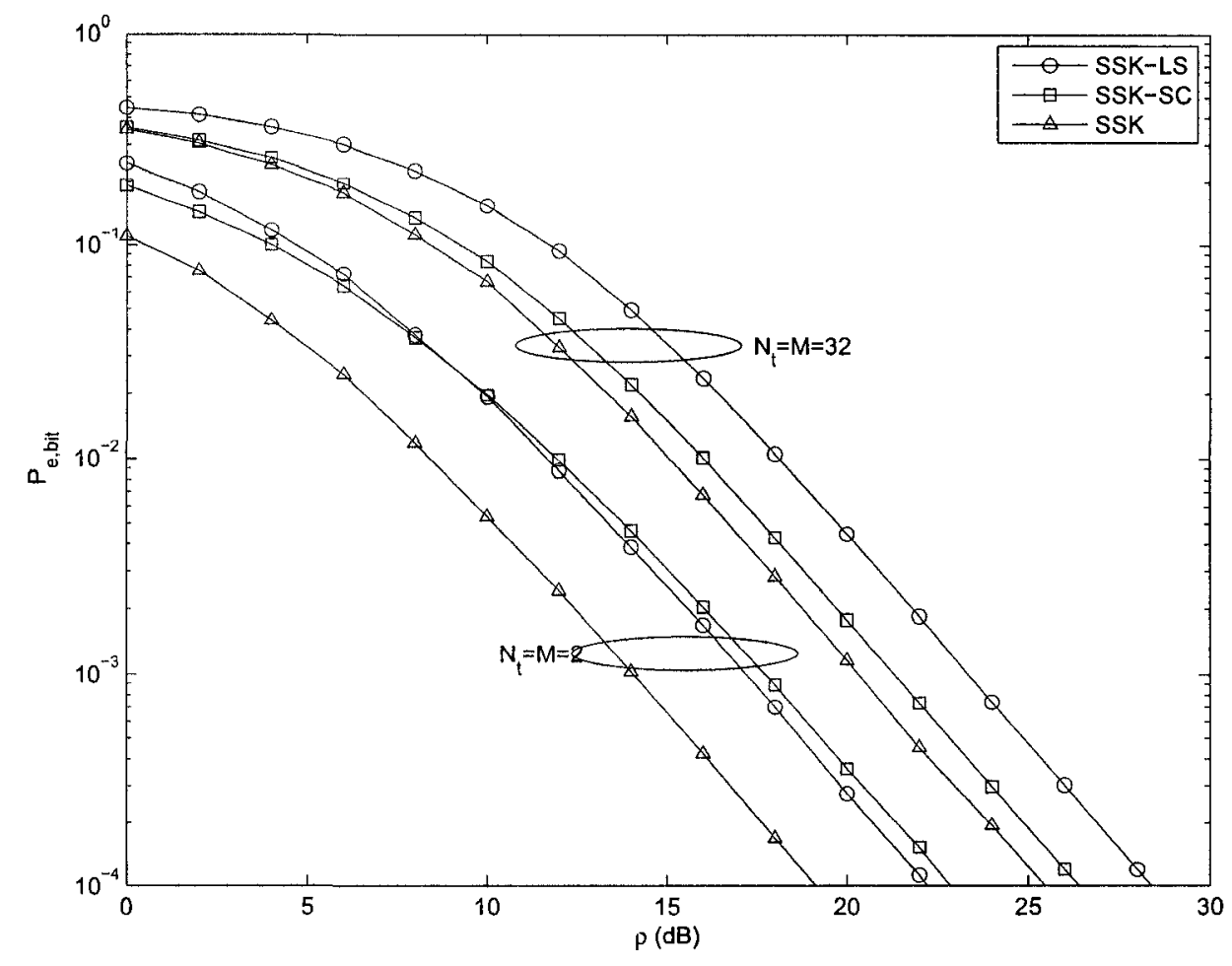

Figure 4.10: BER performance of SSK under non-ideal conditions, for varying $M$ $\left(N_{\mathrm{r}}=2\right)$.

expected. We know from Section 4.1.3 that for increasing $M$ (with $N_{\mathrm{r}}$ fixed), SSK's constellation space becomes more crowded. Therefore, by introducing correlation in an already crowded space (i.e., for $M=32$ ) does not degrade the performance by much. However, for $M=2$, the constellation points are widely spread apart, but adversely affected due to the correlation, and hence, more degradation is observed.

\section{(Non-ideal) GSSK versus SM, and MRC}

In this example, we compare SC effects $\left(\rho_{\text {corr }}=0.1\right)$ on GSSK, SM and MRC performance. We use the same parameters as in Figure 4.4, but incorporate SC within 


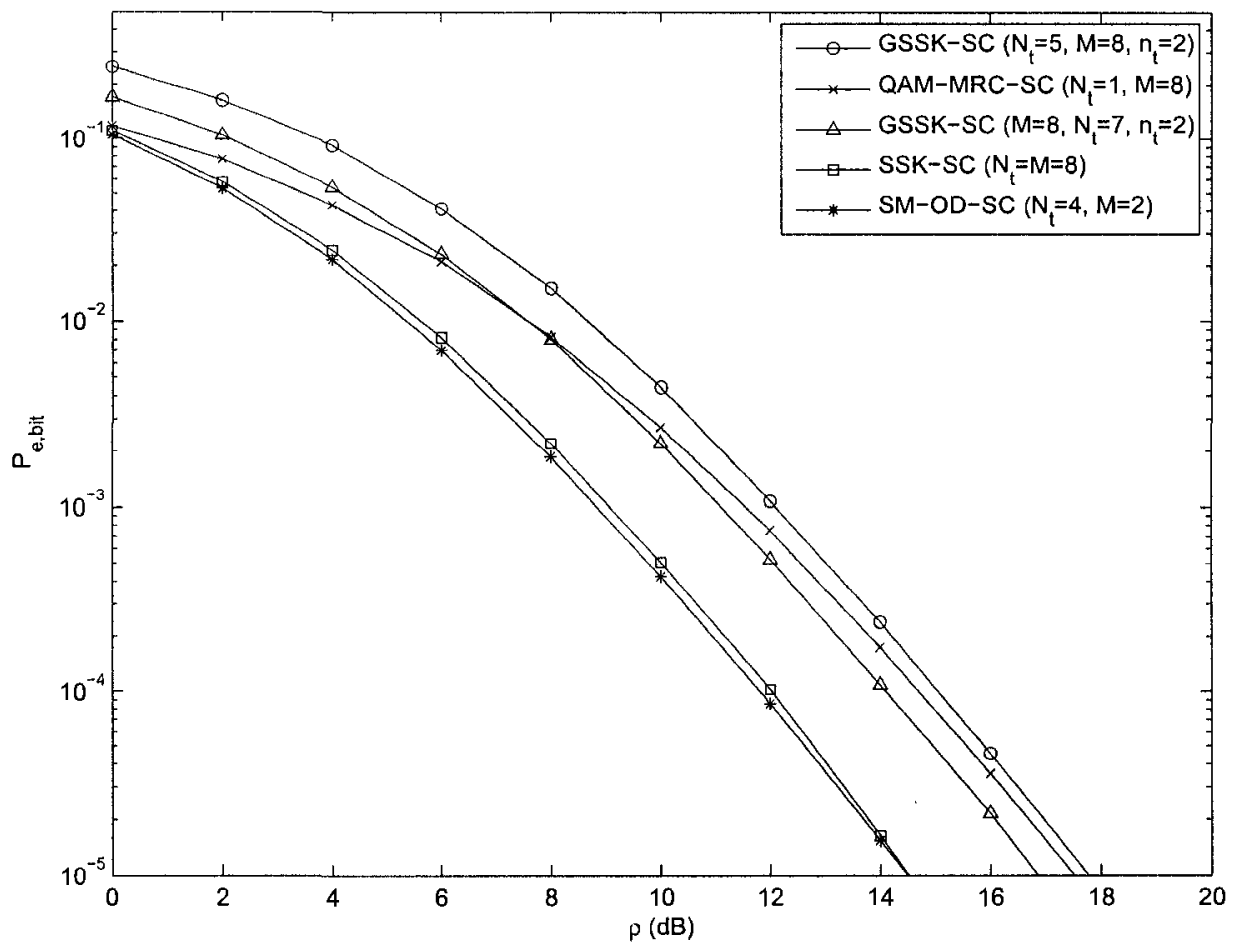

Figure 4.11: BER performance of GSSK versus MRC, and SM, for $m=3 \mathrm{bits} / \mathrm{s} / \mathrm{Hz}$ transmission $\left(N_{\mathrm{r}}=4\right)$ under spatial correlation.

the channel model. From Fig. 4.11, we observe that GSSK degrades similar to SM, and is slightly more sensitive to SC than MRC. In order to overcome such degradation, highly correlated antennas may be grouped to form GSSK constellation points (thus, the set of $M$ constellation points will effectively be less correlated). Such GSSK constellation optimization for correlated channels is a topic under investigation. 


\subsection{Conclusion}

SSK concepts were extensively presented in this chapter. We introduced SSK as a new modulation technique for MIMO communication under fading channels. We presented SSK's constellation, and analyzed the source of SSK's strength to combat fading. A closed form expression for the BER was derived, and extensive simulation results were presented in different scenarios (varying $N_{\mathrm{t}}$ and $N_{\mathrm{r}}$, spatial correlation, channel estimation error). Adaptive SSK was also presented, where antenna selection and adaptive symbol design for SSK was introduced. The results showed performance improvements over MRC and V-BLAST, while performing similar to SM-OD (but requiring lower complexity).

Having provided the fundamentals of SSK for uncoded transmission, we now extend SSK's repertoire to include channel coding. 


\section{Chapter 5}

\section{Coded Space Shift Keying}

CM techniques have been extensively considered in the communications literature, with one of the most significant contributions stemming from Ungerboeck's seminal paper on trellis coded modulation (TCM) [40], where coding and modulation are optimized as a single unit. As an enhancement, turbo CM (TuCM) [41-45] provides performance improvements over TCM by incorporating the turbo principle (turbo codes with iterative decoding). In [46], Zehavi improves TCM's performance in Rayleigh fading channels by separating coding and modulation with a bit-wise interleaver. Caire et al. [47] then extend Zehavi's concept to general constellations, giving rise to BICM systems, which are shown to provide higher diversity gains over conventional TCM systems. In this thesis, we fociss on BICM systems due to these performance advantages, and inherent flexibility of analysis in varying scenarios.

The general system model is similar to the one described in Section 2.1 but with some differences, and is illustrated in Fig. 5.1. A random sequence of independent 
bits $\mathbf{b}=\left[\begin{array}{llll}b_{1} & b_{2} & \cdots & b_{k}\end{array}\right]$ enter a channel encoder with output $\mathbf{c}=\left[\begin{array}{llll}c_{1} & c_{2} & \cdots & c_{n}\end{array}\right]$, where $k$ and $n$ represent the number of encoder inputs and outputs, respectively. The pseudo randomly interleaved sequence $\mathbf{c}^{\pi}$ then enters an SSK mapper, where groups of $m=\log _{2}\left(N_{\mathrm{t}}\right)$ bits are mapped to a constellation vector $\mathbf{x}=\left[\begin{array}{llll}x_{1} & x_{2} & \cdots & x_{N_{\mathrm{t}}}\end{array}\right]^{T}$, with a power constraint of unity (i.e., $E_{\mathbf{x}}\left[\mathbf{x}^{H} \mathbf{x}\right]=1$ ).

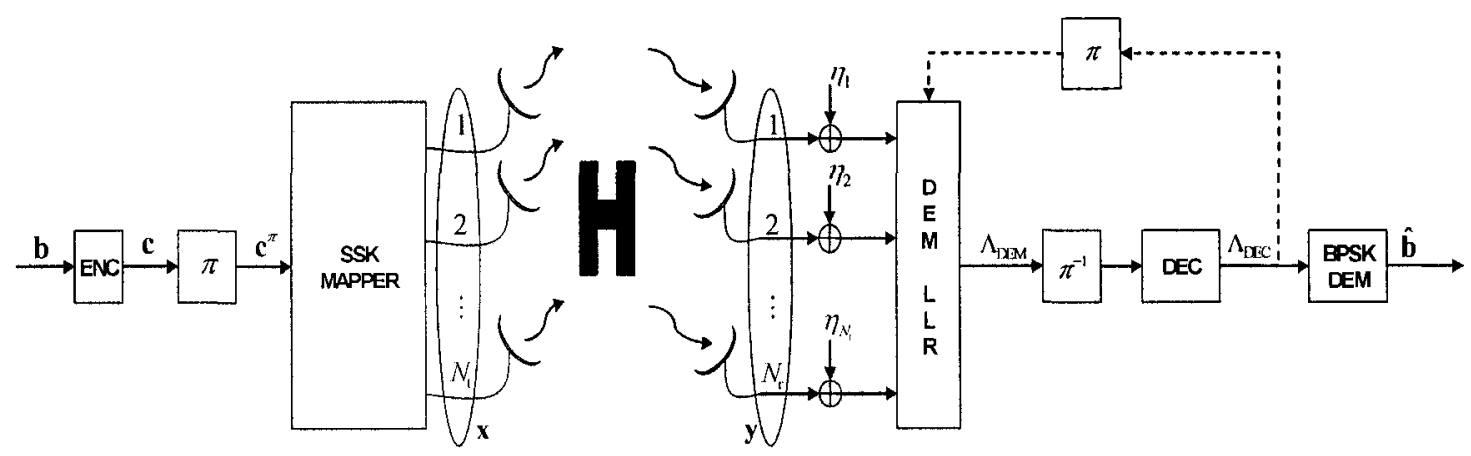

Figure 5.1: SSK-CM system model.

\subsection{Capacity}

The capacity of the system is computed using a generic system model given by Fig.

5.2. The information bits $\mathbf{b}$ enter a channel encoder, followed by a symbol mapper that outputs the modulated sequence $\mathbf{X}=\left\{\mathbf{x}_{1}, \mathbf{x}_{2}, \ldots, \mathbf{x}_{n}\right\}$. The sequence experiences a channel, and the received signal is denoted by $\mathbf{Y}=\left\{\mathbf{y}_{1}, \mathbf{y}_{2}, \ldots, \mathbf{y}_{n}\right\}$. Decoding follows and an estimate of the transmitted bits denoted by $\hat{b}$ is obtained. Our channel model will focus on flat Rayleigh fading with AWGN. 


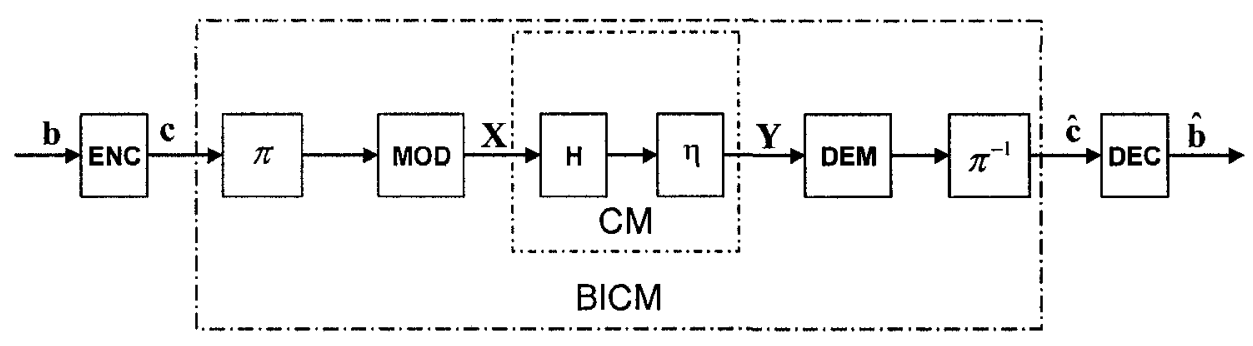

Figıre 5.2: System model for capacity computation.

Assuming $\mathbf{x}$ is transmitted with equal probability (which is justified for an ideally interleaved sequence of channel inputs), the constrained input coded modulation (CM) capacity reduces to $[47]$

$$
C_{\mathrm{CM}}=m-E_{\boldsymbol{\theta}}\left[\log _{2} \frac{\sum_{\boldsymbol{x}^{\prime} \in \mathcal{X}} p_{\mathbf{Y}}\left(\mathbf{y} \mid \mathbf{x}^{\prime}, \mathbf{H}\right)}{p_{\mathbf{Y}}(\mathbf{y} \mid \mathbf{x}, \mathbf{H})}\right],
$$

where $\boldsymbol{\theta}=(\mathbf{x}, \mathbf{y}, \mathbf{H})$, and $p_{\mathbf{Y}}(\mathbf{y} \mid \mathbf{x}, \mathbf{H})$ is given by (4.3). Note that (5.1) is conveniently evaluated by Monte Carlo simulation. The capacity results for SSK modulation with varying $M$ are shown in Fig. 5.3. We would like to compare the capacity of systems requiring (ideally) only one transmit RF chain (similar to an SSK system), and hence, we plot the information rates of PSK and QAM for single antenna transmission. SSK achieves better information rates when $M \geq 8$, and slightly worse for $M<8$. These results are expected since for small $M$ values, APM schemes exploit, enough of the constellation space to achieve adequate performance.

We also plot the BICM capacity (dotted lines), which represents the capacity between the encoder output and the input of the decoder (Fig. 5.2). With some 


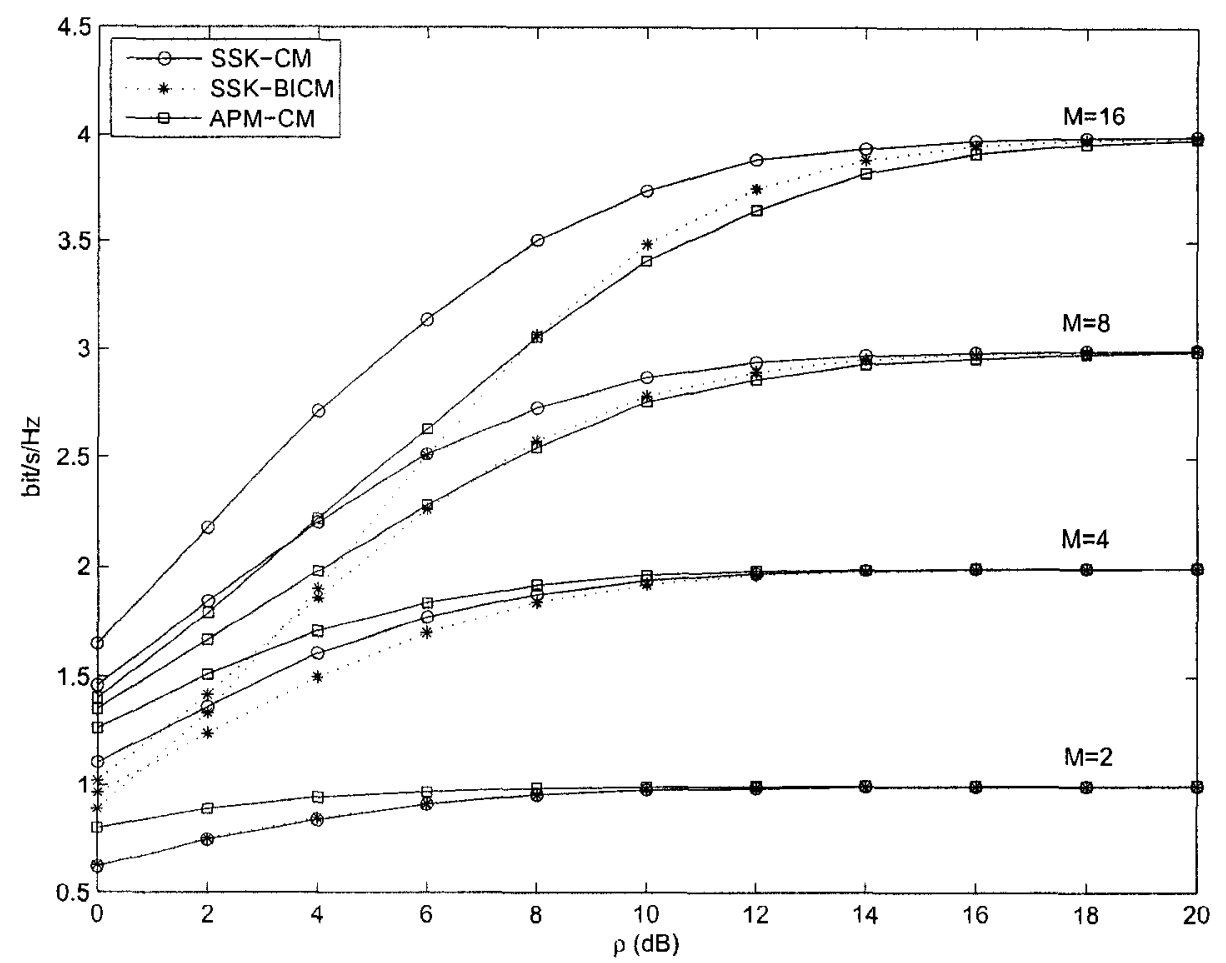

Figure 5.3: SSK constrained input capacity versus SNR, for varying $M\left(N_{\mathrm{r}}=2\right)$.

change of notation, the BICM capacity is given by [47]

$$
C_{\mathrm{BICM}}=m-\sum_{k=1}^{m} E_{\boldsymbol{\theta}}\left[\log _{2}\left(\frac{\sum_{\mathbf{x}^{\prime} \in \mathcal{X}} p_{\mathbf{Y}}\left(\mathbf{y} \mid \mathbf{x}^{\prime}, \mathbf{H}\right)}{\sum_{\mathbf{x}^{\prime} \in \mathcal{X}_{c}^{k}} p_{\mathbf{Y}}\left(\mathbf{y} \mid \mathbf{x}^{\prime}, \mathbf{H}\right)}\right)\right] .
$$

We notice that for increasing $M$, SSK's BICM capacity degrades, especially at low SNRs. This degradation is expected since we did not optimize symbol mapping for the BICM system, as suggested in [47-49]. In fact, SSK uses a different concept 


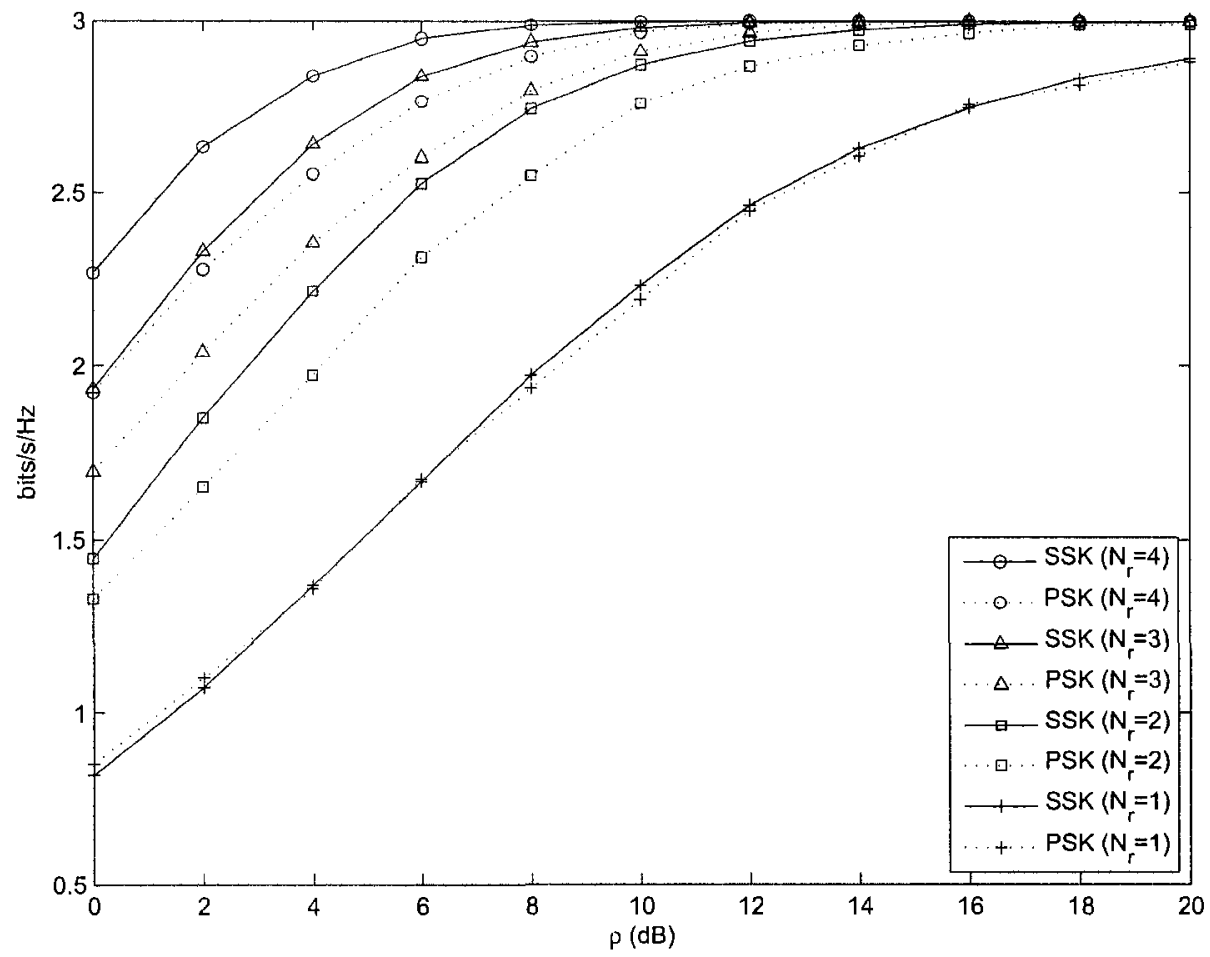

Figure 5.4: SSK constrained input capacity versus SNR, for varying $N_{\mathrm{r}}(M=8)$.

of modulation, where the antennas themselves, and not the symbols, transmit information. Hence, the effective constellation points are random in nature and, without transmitter CSI, we are unable to consider optimal mapping techniques. Regardless, CM (and even BICM with sub-optimal mapping) capacity results suggest SSK performance improvements over APM schemes.

In Fig. 5.4, we vary $N_{\mathrm{r}}$ while fixing the constellation size to $M=8$, and compare the information rates of SSK and PSK. SSK gains more from the increase in $N_{\mathrm{r}}$, especially for the transition of $N_{\mathrm{r}}=1 \rightarrow 2$. For example, a gain of $5 \mathrm{~dB}$ is obtained for SSK at an information rate of 2 bits $/ \mathrm{s} / \mathrm{Hz}$, whereas only $4 \mathrm{~dB}$ is gained for PSK. 
To obtain more insight, and support our capacity results, we analytically derive the performance of SSK-CM in Section 5.3. But first, we describe the structure of SSK-CM, and the adopted decoding method.

\subsection{SSK Coded Modulation}

\subsubsection{Encoding}

Conventional modulation schemes use Gray (or quasi-Gray) mapping to minimize bit errors among symbols. For the case of TCM, bits to symbol mapping is optimized so as to maximize the Euclidean distance between codewords. However, due to these random constellation points inherent in SSK modulation, SSK-TCM is only possible with the transmitter having full channel knowledge. For that reason, conventional CM systems that maximize capacity cannot be considered. Therefore, in our take on SSK-CM, we consider SSK-BICM without any optimized mapping rule. The results of Section 5.1 show severe degradation in SSK-BICM capacity, and so we opt for BICM with iterative decoding (BICM-ID) to bridge the capacity gaps [50].

The encoding is as follows. Information bits are first encoded, bit-wise interleaved, and SSK modulated before transmission. We consider both turbo CM (TuCM) and convolutional CM systems, but there is no restriction on the type of channel code $\mathcal{C}$ used. 


\subsubsection{Decoding}

SSK coded detection employs BICM-ID, and hence uses the decoding structure depicted in Fig. 5.1, proposed by Li and Ritcey in [51]. The demodulator first computes the a posteriori logarithm likelihood ratios (LLR) of the transmitted bits, which are then used as inputs to a channel decoder. Depending on $\mathcal{C}$, we use either a turbo or a maximum a posteriori (MAP) decoder (see [26, Ch. 7] for details). The channel decoder's LLR on the information and parity bits are then processed as extrinsic information in the second iteration of the demodulator's LLR output (shown by the dashed lines in Fig. 5.1). These steps are repeated until a satisfactory level of reliability is obtained (for our case, we consider a fixed number of iterations).

The demodulator LLR is similar to the one given in [45], with some slight modifications, and is given by the following:

$$
\begin{aligned}
\Lambda_{\mathrm{DEM}}(\hat{c}) & =\log \frac{P(\hat{c}=1 \mid \mathbf{y})}{P(\hat{c}=0 \mid \mathbf{y})} \\
& =\log \frac{\sum_{\mathbf{x} \in \mathcal{X}_{1}^{k}} p_{\mathbf{Y}}(\mathbf{y} \mid \mathbf{x}, \mathbf{H}) \prod_{n=1, n \neq k}^{m} P\left(c_{n}\right)}{\sum_{\mathbf{x} \in \mathcal{X}_{0}^{k}} p_{\mathbf{Y}}(\mathbf{y} \mid \mathbf{x}, \mathbf{H}) \prod_{n=1, n \neq k}^{m} P\left(c_{n}\right)}
\end{aligned}
$$

where $p_{\mathbf{Y}}(\mathbf{y} \mid \mathbf{x}, \mathbf{H})$ is given by $(4.3)$, and $P\left(c_{n}=c\right)=\frac{e^{c \Lambda_{\mathrm{DEC}}\left(c_{n}\right)}}{1+e^{\Lambda_{\mathrm{DEC}}\left(c_{n}\right)}}, c \in\{0,1\}$ is obtained from the LLR values of the decoder, $\Lambda_{\mathrm{DEC}}\left(c_{n}\right)$. We employ the same setup for the APM-CM case. 


\subsection{Coded Performance Analysis}

In this section, SSK-CM's analytical performance for fully interleaved channels (each symbol experiences an independent fading matrix) is derived, where, thanks to the binary input-output symmetric (BIOS) property of BICM, the performance analysis is tractable. ${ }^{1}$ The coded system is assumed to be a convolutional code, concatenated with an SSK modulator (as described in Section 5.2), but can be generalized to other concatenated schemes (such as TuCM [52]). We also analyze the diversity of the coded system, and demonstrate interesting diversity improvements over APM-CM for quasi-static channels.

\subsubsection{Coded Error Probability}

The analysis for the coded case is fairly complex, especially due to interleaving and iterative decoding. The simplifying analysis performed in most coding literature regarding the all zero codeword is no longer valid due to the nonlinearity of CM systems. Therefore, similar to [52], we must consider all pairs of codewords to obtain an analytical expression for the probability of error. Most often, however, simple union bound on the BER is used. From $[47,53]$, the bit error probability for BICM under ML decoding is closely upper bounded by

$$
P_{\mathrm{e}, \mathrm{bit}} \leq\left. B(X)\right|_{X=[\operatorname{PEP}(d, \mu, \mathcal{X}, \rho)]^{\frac{1}{d}}},
$$

\footnotetext{
${ }^{1}$ For signal constellations $\mathcal{X}$ leading to a non-symmetric BICM channel, a method to symmetrize the channel is presented in [47].
} 
where $B(X) \triangleq \sum B_{d} X^{d}, B_{d}=\sum_{i} \frac{i}{k} A_{i, d}$ ( $A_{i, d}$ represents the number of codewords in $\mathcal{C}$ with output Hamming weight $d$ and input weight $i$ ).

In order to further simplify the analysis, we incorporate the LLR within the derivation as in [47]. We therefore require the statistical properties of the LLR random variable. Specifically, we need a closed form expression for the PDF of the LLR, $p_{\Lambda}(\lambda)$. These PDFs are obtained in [54] for APM, but in the case of multi-dimensional constellations, a closed form expression is difficult to derive. To overcome this challenge, [55] presents simple upper bounds based on the saddlepoint (SP) approximation, without explicitly obtaining $p_{\Lambda}(\lambda)$. The BICM PEP is therefore upper bounded using the SP approximation for fully interleaved channels as [55]

$$
\operatorname{PEP}_{\mathrm{BICM}}(d, \mu, \mathcal{X}, \rho) \approx \frac{\exp (d \tau(\hat{s}))}{\sqrt{2 \pi d \tau^{\prime \prime}(\hat{s})} \hat{s}}
$$

where $\tau(\hat{s})$ is the cumulant generating function of the random variable $\Lambda_{\mathrm{DEM}}$ defined in $(5.2), \tau^{\prime \prime}(\hat{s})$ represents its second derivative, and $\hat{s}=\frac{1}{2}$ for BIOS channels. Equation (5.4) is plugged into (5.3) to obtain an upper bound on $P_{\text {e,bit }}$. We note that (5.4) is conveniently evaluated using Monte Carlo simulation, or numerical integration methods with Gaussian quadrature rules (see [55] for details).

Equation (5.4) does not, however, take into consideration the effects of iterative decoding, which is the type of decoder employed in this thesis. Hence, (5.4) is strictly an upper bound for the BICM-ID case. In order to make fair comparisons, we derive the asymptotic BICM-ID bounds (valid after convergence, i.e., for sufficiently high 
SNRs and large number of decoding iterations), also referred to as the error-free feedback performance [56]. The assumption of error-free feedback implies that each bit is transmitted using an equivalent BPSK type system. Consequently, we can directly apply the results of [57], and obtain closed form PEP bounds on error-free feedback. Noting that for SSK, $\Lambda_{\mathrm{DEM}} \sim \mathcal{N}\left(-4 N_{\mathrm{r}}^{-1} \kappa_{\mathrm{SSK}}, 8 N_{\mathrm{r}}^{-1} \kappa_{\mathrm{SSK}}\right)$, where $\kappa_{\mathrm{SSK}}$ is obtained from (4.6), we obtain the saddlepoint approximation for the PEP as [57, Eq.

$$
\operatorname{PEP}_{\text {BTCM-ID }}(d, \mu, \mathcal{X}, \rho) \approx \frac{1}{2 \sqrt{\pi d \rho}}\left(1+\frac{\rho}{N_{\mathrm{r}}}\right)^{-d N_{\mathrm{r}}+\frac{1}{2}}
$$

Remark 7 Interestingly, the error-free feedback bounds for SSK are independent of the constellation size. Hence, SSK-CM's asymptotic performance is identical for all $M$. This is not the case for APM-CM, where the performance degrades as $M$ is increased. This degradation is due to the decrease in average Euclidean distance between. equivalent BPSK symbols (obtained from the constellation of size M). However, the equivalent BPSK symbols in SSK will have, on average, the same Euclidean distance regardless of $M$.

\subsubsection{Coded Diversity}

Coded diversity for the APM-CM case is easily understood, where experiencing different fading coefficients within a codeword increases the diversity order, and hence the reliability. However, for SSK-CM, the fading columns themselves relay information, 
and the diversity consideration is not as straightforward. Here, we present an intuitive explanation of SSK-CM's diversity effect, in both fully interleaved and quasi-static channels.

Assume two space time codewords $\mathbf{X}_{1}$ and $\mathbf{X}_{2}$ of length $L$, where $\mathbf{X}=\left[\begin{array}{llll}\mathbf{x}_{1} & \mathbf{x}_{2} & \cdots & \mathbf{x}_{L}\end{array}\right]$, and $\mathbf{x}$ is as defined in Section $4.1 .^{2}$ The PEP, conditioned on $\mathbf{H}$, for ML detection is given by $[26$, p. 97]

$$
P\left(\mathbf{X}_{1} \rightarrow \mathbf{X}_{2} \mid \mathbf{H}\right)=Q\left(\sqrt{\frac{1}{2} \rho\left\|\mathbf{D}_{21}\right\|_{\mathrm{F}}^{2}}\right)
$$

where $\mathbf{D}_{21}=\mathbf{H}\left(\mathbf{X}_{2}-\mathbf{X}_{1}\right)$. Say the codewords $\mathbf{X}_{1}$ and $\mathbf{X}_{2}$ differ only in $d\left(\mathbf{X}_{1}, \mathbf{X}_{2}\right)$ places. Let $\mathbf{X}^{\prime}=\left[\begin{array}{llll}\mathbf{x}_{1}^{\prime} & \mathbf{x}_{2}^{\prime} & \cdots & \mathbf{x}_{d\left(\mathbf{x}_{1}, \mathbf{X}_{2}\right)}^{\prime}\end{array}\right]$ denote the $d\left(\mathbf{X}_{1}, \mathbf{X}_{2}\right)$ terms contributing to the conditional PEP, and let us define a difference matrix as $A^{\prime}=\left(\mathbf{X}_{2}^{\prime}-\mathbf{X}_{1}^{\prime}\right)$.

Diversity: We obtain diversity if the component constellation vectors of $\mathbf{X}^{\prime}$ (i.e., the $\mathbf{x}^{\prime} \mathbf{s}$ ) activate different colımns of $\mathbf{H}$. We illustrate this diversity effect in Fig. 5.5 by considering the all zero codeword, and a possible error event on the trellis (for $M=4$ ). The error event in the figure traverses two distinct symbol outputs, hence two distinct columns of $\mathbf{H}$ (which contributes to diversity). We also sketch a possible realization for $\mathbf{H}$ (i.e., the effective constellation space $\mathcal{X}^{\text {eff }}$, where $n_{\text {neigh }}$ represents the number of nearest neighbors. By having different symbols in the trellis path, there is a better chance that the distance property for a particular column of $\mathbf{H}$ is more reliable (such as the case for $\mathbf{h}_{2}$ in Fig. 5.5).

In fully interleaved channels, both SSK-CM and APM-CM systems achieve this

\footnotetext{
${ }^{2}$ No assumption is made on the type of modulation.
} 


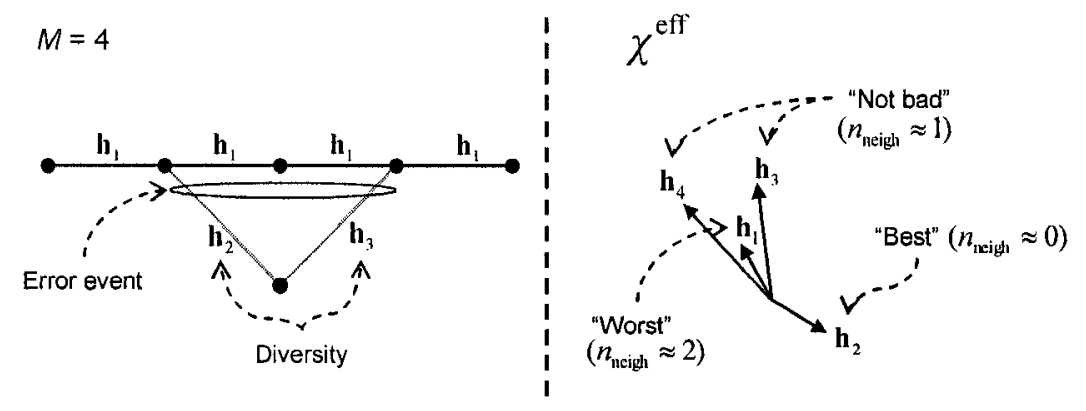

Figure 5.5: Ilustration of SSK-CM's diversity in quasi-static fading channels.

aspect of diversity (i.e., a diversity of $\left.d\left(\mathbf{X}_{1}, \mathbf{X}_{2}\right) N_{\mathrm{r}}\right)$. However, for quasi-static fading channels, APM only experiences one column of $\mathbf{H}$, and there is only receive diversity (i.e., a diversity of $N_{\mathrm{r}}$ ). In SSK-CM, a diversity order equal to the number of distinct symbols in $A^{\prime}$ is achieved, which ranges between $N_{\mathrm{r}}$ (the diversity of uncoded transmission) and $\min \left(M, d\left(\mathbf{X}_{1}, \mathbf{X}_{2}\right)\right) N_{\mathrm{r}}$, with a maximum equal to the fully-interleaved diversity.

Remark 8 Asymptotically, the coded performance is not only affected by the shortest error event in the trellis, but also the number of distinct symbols in. $A^{\prime}$. For example, it is possible to have a short error event with large diversity order, and a large error event with small diversity order. Therefore, a good code $\mathcal{C}$ for SSK-CM should be optimized taking into consideration both $d\left(\mathbf{X}_{1}, \mathbf{X}_{2}\right)$, and the diversity order achievable by $A^{\prime}$. To improve performance, codes may be designed to exploit this diversity property. 


\subsection{Simulation Results}

In this section, we present some examples to compare SSK's coded performance in different channel conditions. Monte Carlo simulations are performed in both fully interleaved and quasi-static environments, and are run for at least $10^{5}$ channel realizations. The plots illustrate the average BER performance versus $\rho$, where Gray or quasi-Gray mapping is used when appropriate (i.e., for PSK or QAM modulation). The receiver is assumed to be the iterative demodulator-decoder from Fig. 5.1, where we consider two decoding iterations (i.e., one pass of the feedback loop), unless stated otherwise. For CM, we consider a convolutional CM system using a rate $\frac{1}{2}$ convolutional encoder given by the generator matrix $G=\left[\begin{array}{ll}1 & \frac{1+D^{2}}{1+D+D^{2}}\end{array}\right]$. As for the TuCM system, We use a rate $\frac{1}{3}$ turbo code composed of two, rate $\frac{1}{2}$ convolutional encoders given by $G$, unless stated otherwise.

\subsection{1 (Quasi-Static) SSK-CM versus APM-CM (varying $M$ )}

We first investigate the effects of higher order modulation on the SSK-CM system, and compare them to APM-CM (either QAM or PSK) for quasi-static environments. From Fig. 5.6, we observe additional diversity order for SSK-CM over APM-CM, especially for increasing $M$ (as predicted in Section 5.3.2). Since the channel is quasistatic, the codewords for the APM-CM case only observe one channel realization from the transmitter side. Hence, the total diversity available is equal to the number of receive antennas $N_{\mathrm{r}}$. In contrast, the codewords for the SSK-CM system will 


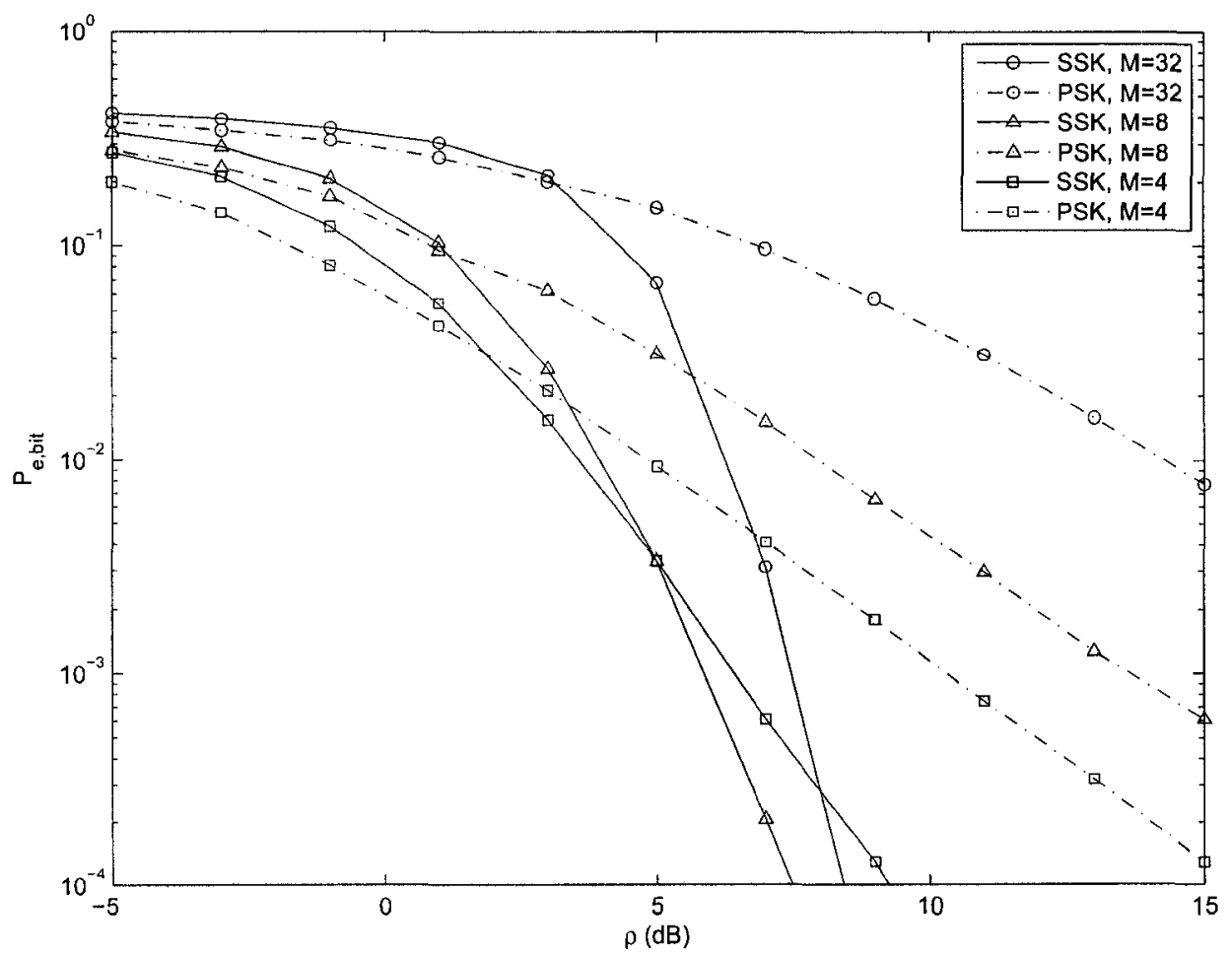

Figure 5.6: BER performance of SSK-CM versus PSK-CM, for varying $M\left(N_{\mathrm{r}}=2\right)$ in quasi-static channels.

experience different channel realizations. Therefore, the potential for SSK diversity gain is possible. As illustrated in the results of Fig. 5.6, the APM-CM scheme achieves a diversity of $N_{\mathrm{r}}=2$ for all cases, whereas the SSK-CM system achieves a diversity order greater than or equal to $N_{\mathrm{r}}=2$.

\subsection{2 (Quasi-Static) SSK-CM versus APM-CM (varying $N_{\mathrm{r}}$ )}

In Fig. 5.7, we present the effects of $N_{\mathrm{r}}$ on coded SSK. We set $M=8$, and compare the results with PSK. SSK obtains large gains through diversity enhancements from $N_{\mathrm{r}}$ and $M$, whereas APM only receives diversity from $N_{\mathrm{r}}$. SSK gains about $7 \mathrm{~dB}$ over 


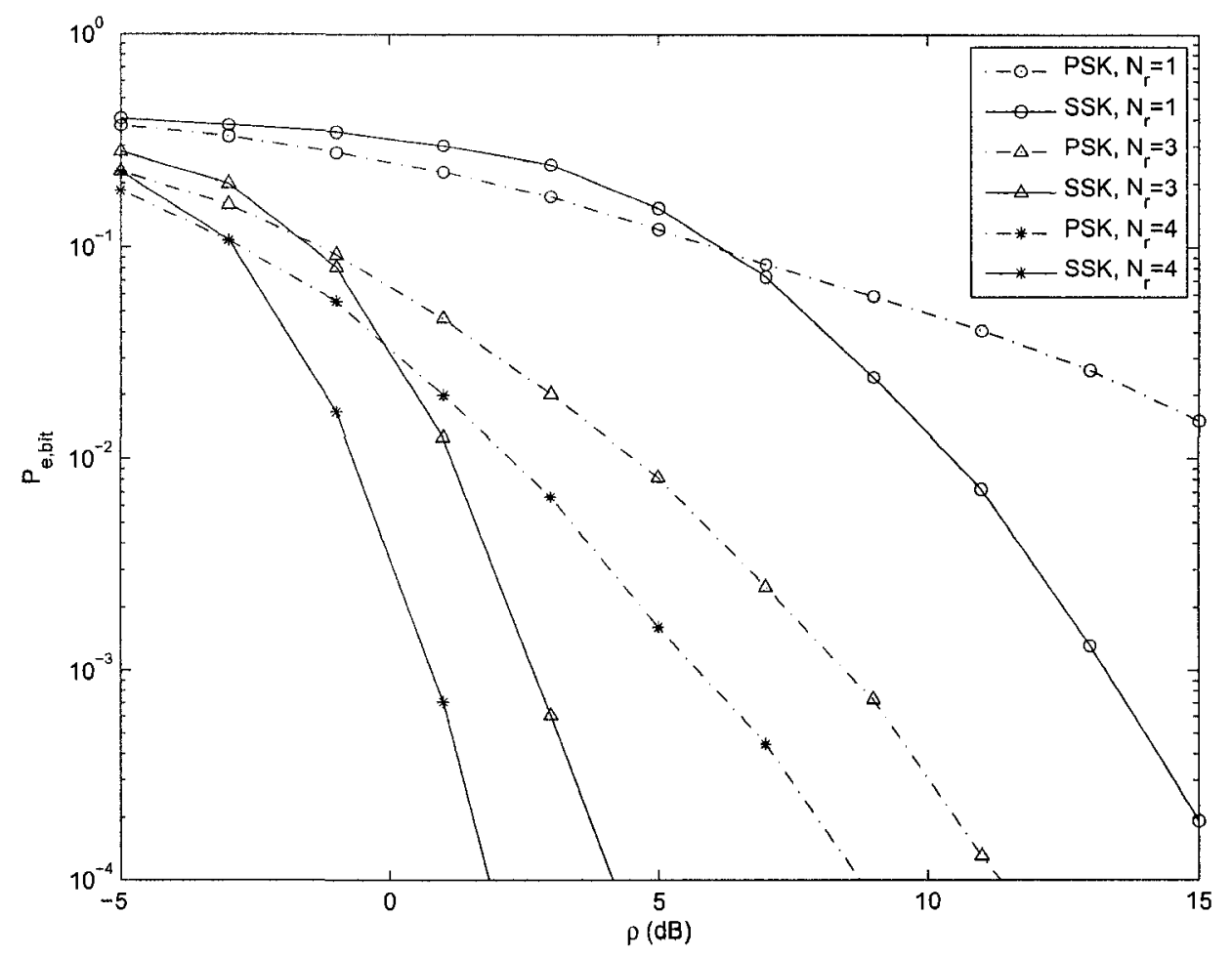

Figure 5.7: BER performance of SSK-CM versus PSK-CM, for varying $N_{\mathrm{r}}(M=8)$ in quasi-static channels.

PSK at $P_{\text {e,bit }}=10^{-4}$, for $N_{\mathrm{r}}=4$.

\subsection{3 (Quasi-Static) SSK-TuCM versus APM-TuCM (vary- ing $M$ )}

We now investigate the effects of higher order modulation on the SSK-TuCM system, and compare them to PSK-TuCM for quasi-static environments (we consider two turbo decoding iterations). From Fig. 5.8, we observe additional diversity order in SSK-TıCM over PSK-TuCM, especially for increasing $M$ (as predicted in Section 


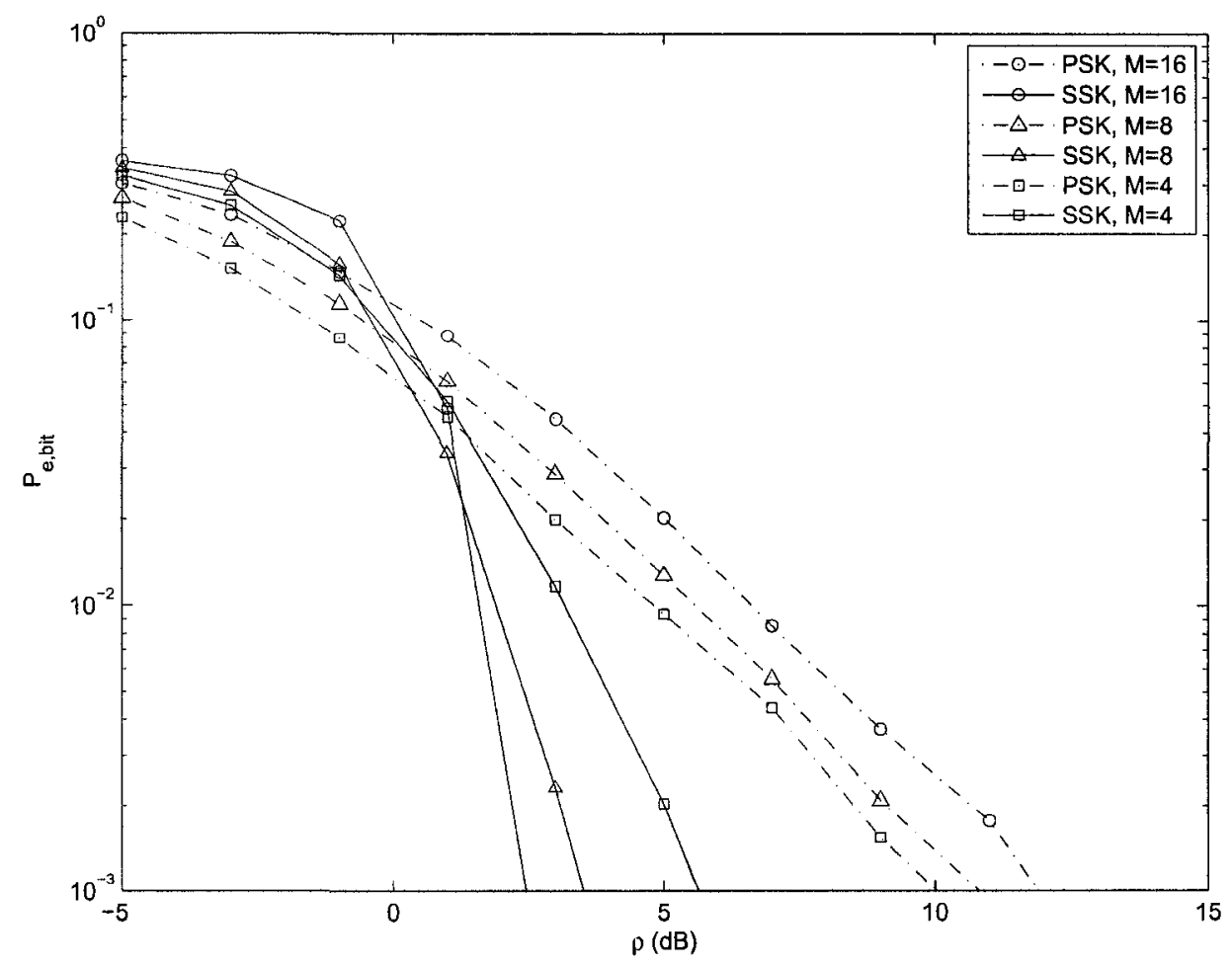

Figure 5.8: BER performance of SSK-TuCM versus PSK-TuCM, for varying $M\left(N_{\mathbf{r}}=\right.$ 2) in quasi-static channels.

5.3.2). PSK-TiCM achieves a diversity of $N_{\mathrm{r}}=2$ for all cases, whereas the SSKTuCM system achieves a diversity order greater than or equal to $N_{\mathrm{r}}=2$. Gains of about, $9 \mathrm{~dB}$ are observed at $P_{\mathrm{e}, \mathrm{bit}}=10^{-3}$, for $M=16$.

\subsection{4 (Quasi-Static) SSK-TuCM versus APM-TuCM (vary- ing $N_{\mathrm{r}}$ )}

In Fig. 5.9, we present the effects of $N_{\mathrm{r}}$ on SSK-TuCM. We set $M=8$, and compare the results with PSK. SSK obtains large gains through diversity enhancements from 


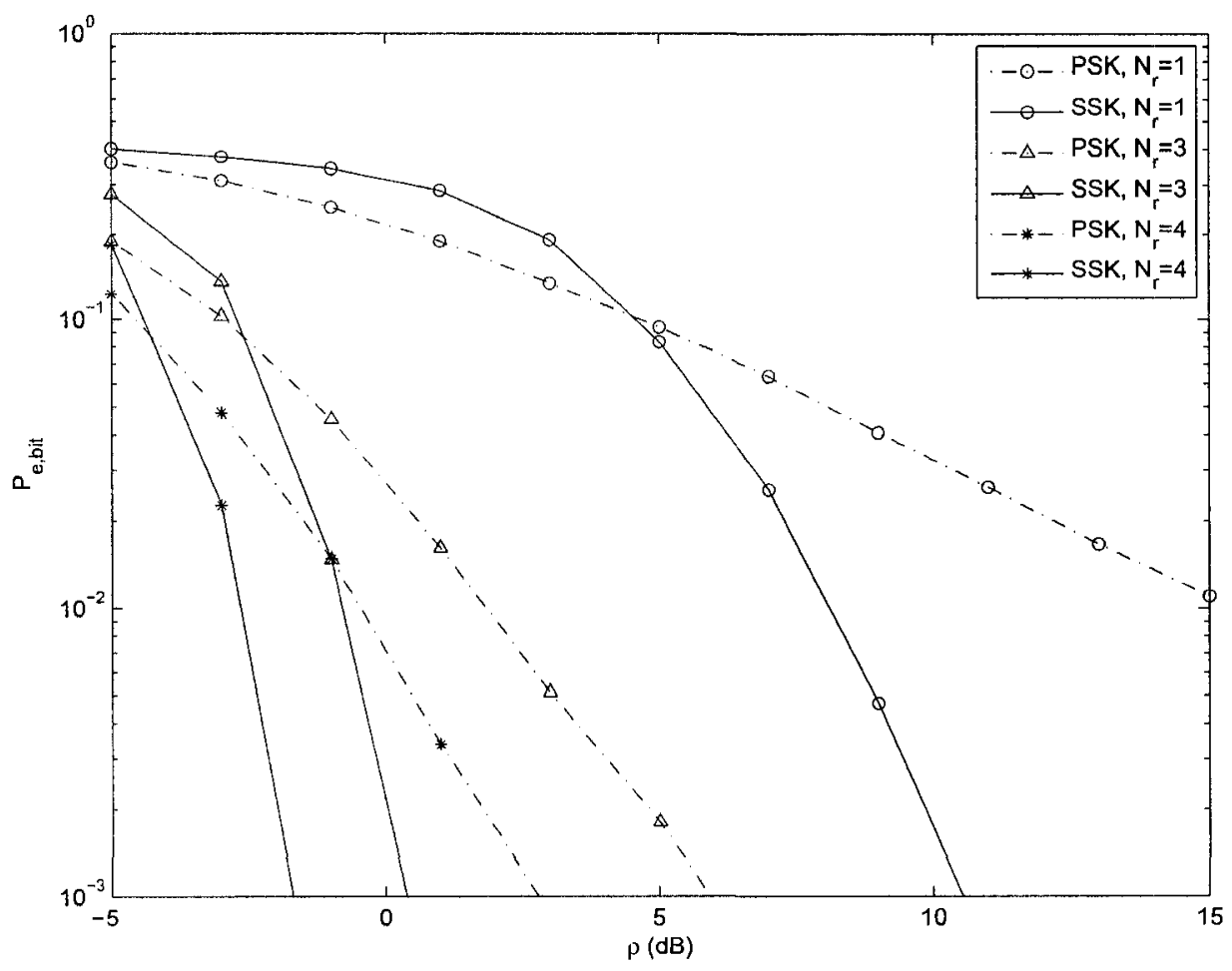

Figure 5.9: BER performance of SSK-TuCM versus PSK-TuCM, for varying $N_{\mathrm{r}}(M=$ 8) in quasi-static channels.

$N_{\mathrm{r}}$ and $M$, whereas APM only achieves diversity from $N_{\mathrm{r}}$. SSK gains about $7 \mathrm{~dB}$ over PSK at $P_{\mathrm{e}, \mathrm{bit}}=10^{-2}$, for $N_{\mathrm{r}}=1$.

\subsection{5 (Fully Interleaved) SSK-CM versus APM-CM (varying}

M)

Figures 5.10 and 5.11 illustrate SSK-CM's performance for $M=8$ and $M=16$, respectively. For fully interleaved channels, both SSK and APM have the same diversity advantage (Section 5.3.2), but with SSK still outperforming PSK $(M=8)$ and QAM 


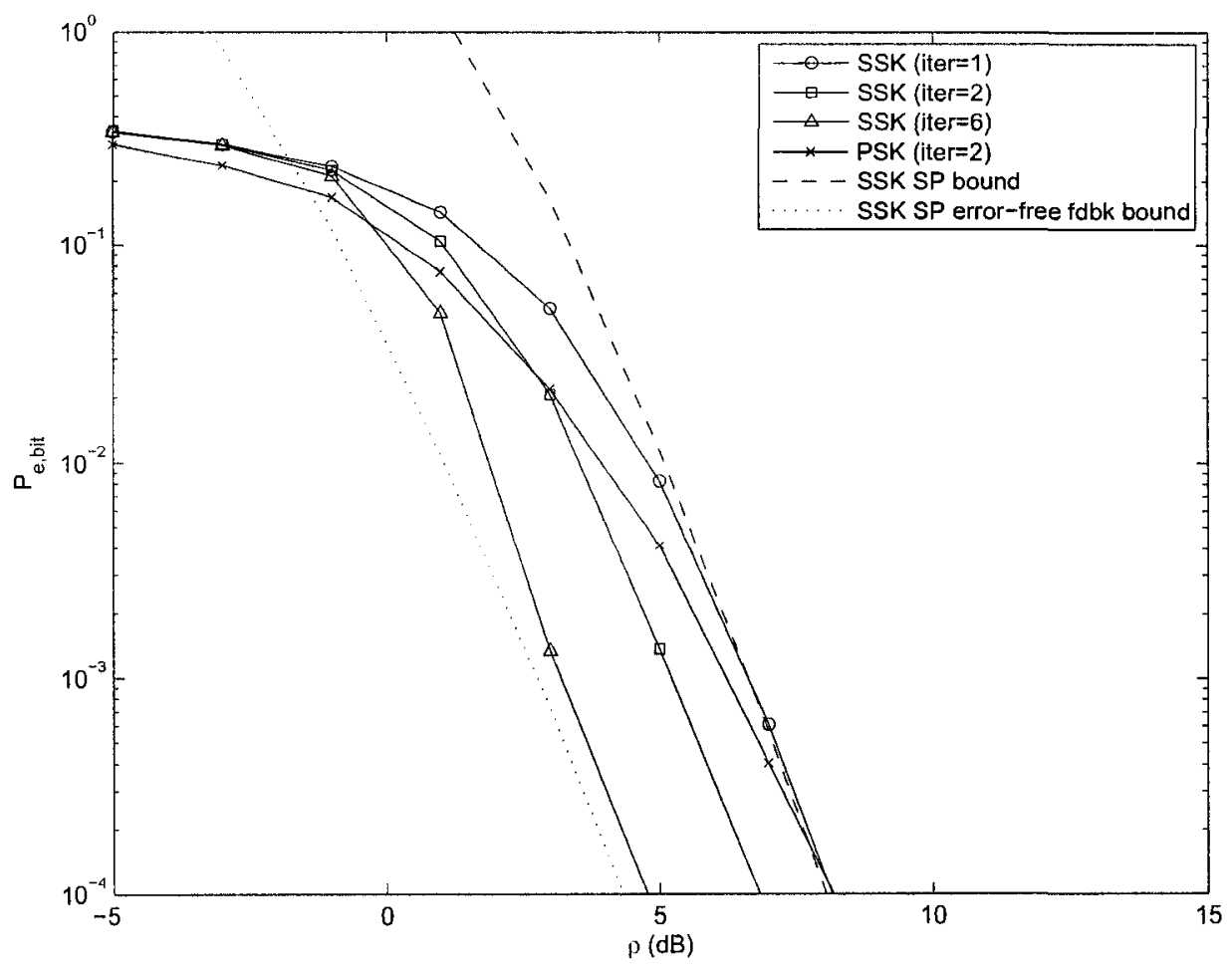

Figure 5.10: BER performance of SSK-CM versus PSK-CM, for vaying number of iterations $\left(M=8, N_{\mathrm{r}}=2\right)$ in fully interleaved channels.

$(M=16)$. We also plot SSK's SP bounds on BER, using the BICM PEP of (5.4), and the error-free feedback PEP of $(5.5)$, with $B(X)=\frac{3 X^{5}-6 X^{6}+2 X^{7}}{1-4 X+4 X^{2}}[53$, p. 504]. To validate the obtained bounds, we simulate SSK-CM for one decoding iteration (i.e., BICM without ID), and six decoding iterations (i.e., asymptotic BICM-ID). We observe that the BICM bounds are fairly tight, especially at high SNRs, while the error-free feedback bounds denote a lower limit on SSK-BICM-ID's performance. 


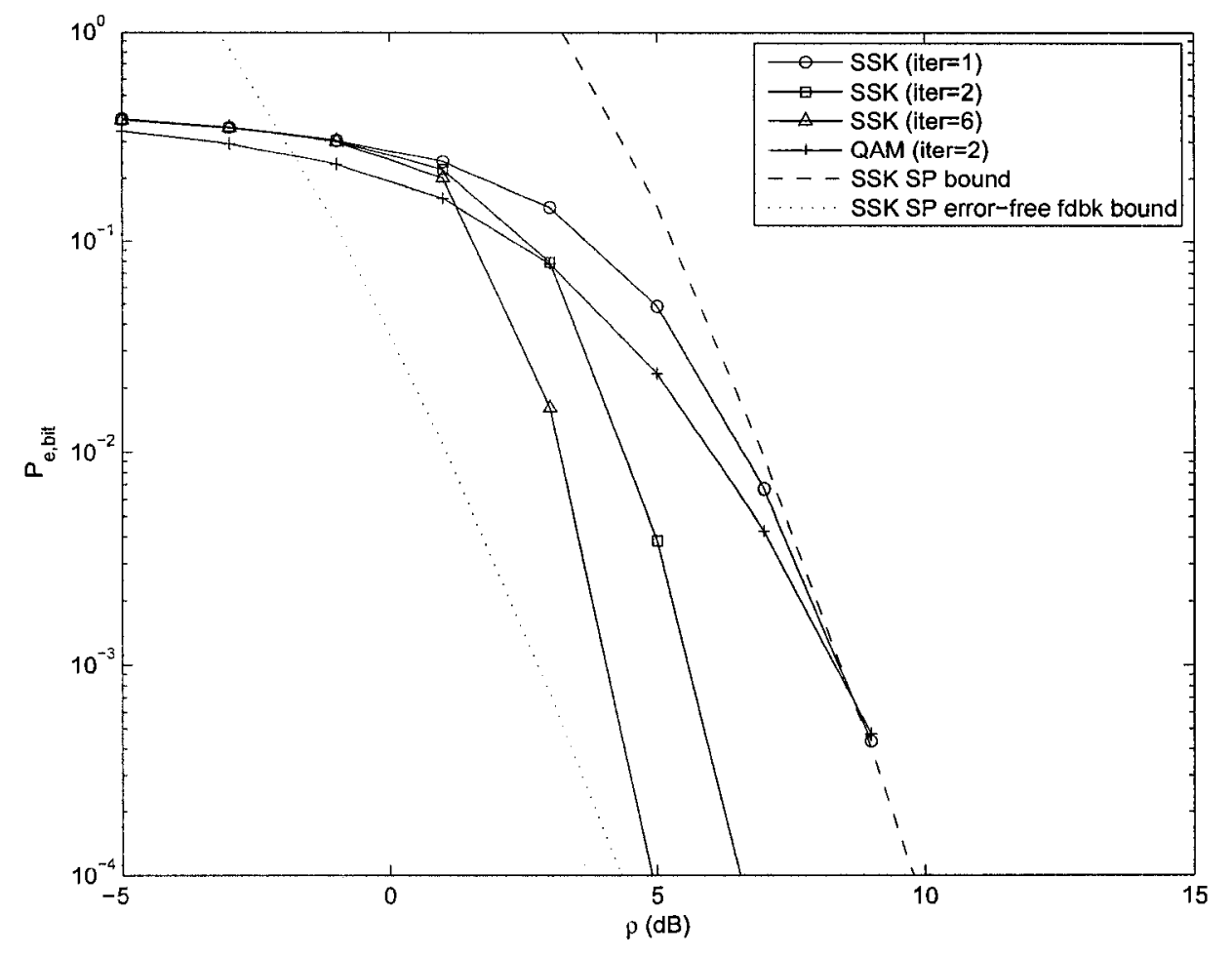

Figure 5.11: BER performance of SSK-CM versus QAM-CM, for vaying number of iterations $\left(M=16, N_{\mathrm{r}}=2\right)$ in fully interleaved channels.

\subsection{6 (Fully Interleaved) SSK-TuCM versus APM-TuCM (vary-}

$$
\text { ing } M \text { ) }
$$

In this example, we obtain performance results for higher rates and hence use a rate $\frac{3}{4}$ punctured turbo code composed of two, rate $\frac{1}{2}$ convolutional encoders given by the 
generator matrix $G=\left[\begin{array}{ll}1 & \frac{1+D+D^{2}+D^{3}}{1+D^{2}+D^{3}}\end{array}\right]$. We consider a puncturing pattern given by

$$
P=\left[\begin{array}{llllll}
1 & 1 & 1 & 1 & 1 & 1 \\
1 & 0 & 0 & 0 & 0 & 0 \\
0 & 0 & 1 & 0 & 0 & 0
\end{array}\right]
$$

and use one turbo decoding iterations for each demodulator-decoder iteration. The first row of $P$ represents the information bit sequence (where all of them are kept), and the second and third rows represent the first and second parity sequences. That is, the second row signifies that the first parity bit will be kept and five will be punctured from the first parity sequence, whereas only the third parity bit will be kept in the second parity sequence.

Figures 5.12 and 5.13 illustrate SSK-TuCM's performance for $M=8$ and $M=16$, respectively. Iterations for APM-TuCM are also shown, where improvements are achieved due to more turbo decoder passes. SSK is shown to outperform APM by 1.5 $\mathrm{dB}$ and $2 \mathrm{~dB}$ for $M=8$ and 16 , respectively $\left(P_{\mathrm{e}, \mathrm{bit}}=10^{-4}\right)$. As well, the results are relatively close to capacity. For example, the results for 8 -SSK tend to $P_{\mathrm{e}, \mathrm{bit}}=10^{-5}$ at about $7 \mathrm{~dB}$, which is $2 \mathrm{~dB}$ away from capacity (i.e., $\frac{9}{4} \mathrm{bit} / \mathrm{s} / \mathrm{Hz}$ is achieved at around $5 \mathrm{~dB}$ ). For $16-\mathrm{SSK}$, the results tend to $P_{\mathrm{e}, \mathrm{bit}}=10^{5}$ at about $8.5 \mathrm{~dB}$, which is about $3 \mathrm{~dB}$ away from capacity. 


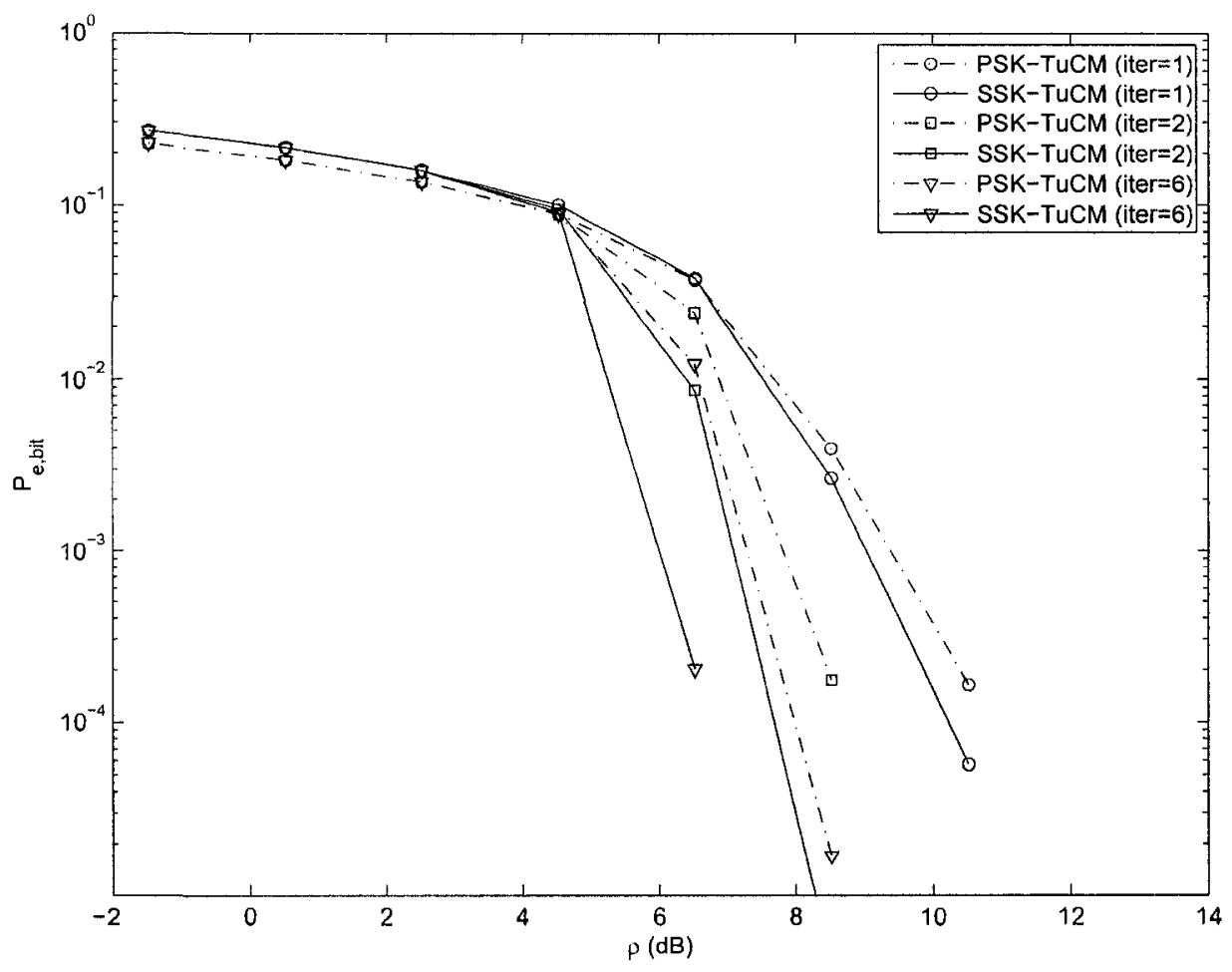

Figure 5.12: BER performance of SSK-TuCM versus PSK-TuCM, for vaying number of iterations $\left(M=8, N_{\mathrm{r}}=2\right)$ in fully interleaved channels.

\subsection{Conclusion}

The coded SSK system was presented in this chapter. We provided capacity results, and illustrated gains over APM systems. The BER of coded SSK was upper bounded, and we provided an analysis of SSK's diversity advantage over APM. Extensive simulation results were shown to support, our analysis, in both quasi-static and fast fading environments. 


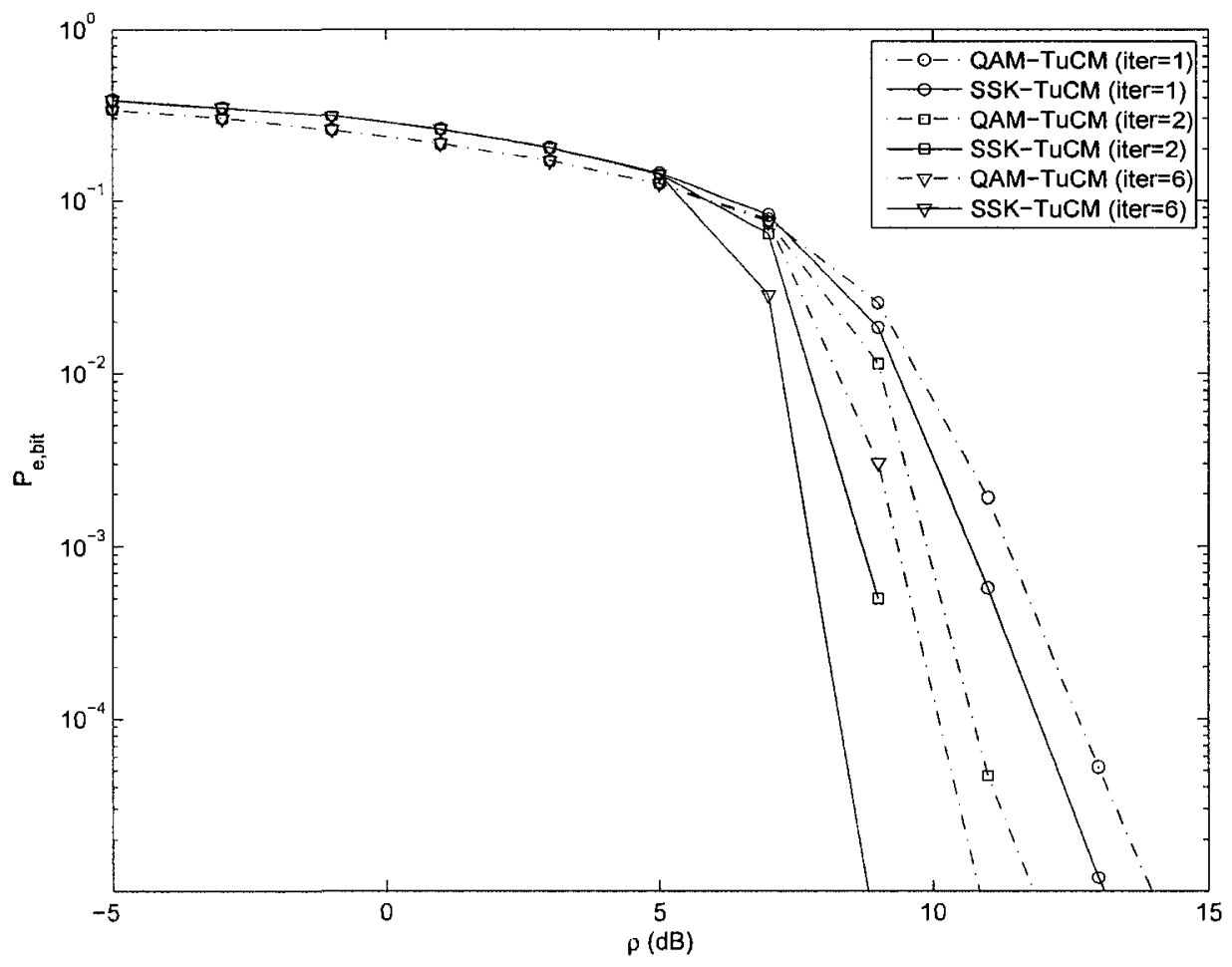

Figure 5.13: BER performance of SSK-TuCM versus QAM-TuCM, for vaying number of iterations ( $\left.M=16, N_{\mathrm{r}}=2\right)$ in fully interleaved channels. 


\section{Chapter 6}

\section{Conclusion and Future Work}

\subsection{Concluding Remarks}

In this thesis, we derived the optimal SM detector for which, significant performance gains were observed over the detector in [22]. To support our results, we also derived a closed form expression bounding the average BER of SM when real constellations were used. The simulation results indicated that SM with optimal detection outperformed V-BLAST and APM transmission, providing another promising candidate for low complexity transmission techniques.

As the main focus of the thesis, we introduced a modulation technique (referred to as SSK) for MIMO wireless links, by exploiting the inherent fading process. Rather than transmitting information through symbols, the transmitter antenna index was used as the sole information relaying mechanism, where only one antenna was kept, active during a transmission period (hence, ideally only $1 \mathrm{RF}$ chain being needed). 
Throughout the thesis, we laid out SSK fundamentals as the building ground for hybrid modulation schemes (which combine SSK and APM) such as in $[9,19,21,23]$. We derived closed form upper bounds on the bit error probability, and used them to build adaptive forms of SSK as a means to improve performance. We also presented SSK-CM, which was shown to achieve higher capacity results than APM-CM. We derived closed form upper bounds on the bit error probability of the coded system, and discovered SSK's significant diversity advantage over APM (in quasi-static channels). Large performance gains were also shown over APM-CM (up to $9 \mathrm{~dB}$ ).

All of SM's merits mentioned in [23] are also inherent in SSK (at similar performance), but with lower computational overhead, and with relaxed APM hardware requirements. These advantages make SSK an interesting candidate for low complexity transceivers in next generation communication systems.

\subsection{Future Research}

In our efforts to introduce and analyze SSK concepts, we bore in mind to begin our research with the fundamentals. We chose to study SSK in simple, and ideal scenarios, in order to build strong foundations for its growth. However, we always foresaw SSK's practical implementation within wireless communication's discipline, and are excited to extend SSK's knowledge base. The next few sections lists some attractive extensions for further research. 


\subsubsection{Practical implementation issues}

Throughout the thesis, we mentioned some practical issues with SSK, and often suggested potential solutions. One main area of concern is the requirement for SSK to have $N_{\mathrm{t}}=M$ transmit antennas. We introduced GSSK as method to combat this problem, and also mentioned that since SSK only transmits on one antenna at any given time, ideally only one RF chain was required. Therefore, although the SSK system would require a large number of antennas, the main bottleneck involved with multiple antennas (the RF chains) is fixed. This issue needs practical consideration, and requires more research. In particular, pulse shaping for band-limited channels usually result in signals occupying multiple symbol periods. The consequence of pulse shaping is 1) antennas cannot simply be switched "off" and hence, 2) there will be significant interference amongst antennas, since SSK detection is done on a symbol by symbol basis.

One potential method for overcoming this pulse shaping issue is to use two RF chains in conjunction: one for activating antennas, and the other to transition between antennas. This would eliminate the switching "off" problem. However, methods to overcome the interference of the two antennas being somewhat activated at the same time remains an open research problem.

\subsubsection{Symbol Design}

In Section 4.3.1, we presented one optimal symbol design method for SSK, and illustrated great improvement over the non-adaptive case. However, for $N_{\mathbf{r}}>1$, methods 
to optimally design SSK's transmit symbols was not pursued, and is an interesting area of research. Also, our optimal design required full transmit, channel knowledge, and hence was not very practical. Extensions to limited feedback adaptive design (such as [33]) is also an interesting topic for research.

As well, we mentioned in Section 5.1 that we did not optimize symbol mapping for the BICM system, resulting in more degraded performance compared to a CM system. However, with full CSI, such symbol mapping optimization is possible.

\subsubsection{Diversity Codebook Design}

Asymptotically, we showed that the coded performance is not only affected by the shortest error event in the trellis, but also the number of distinct symbols in $A^{\prime}$ (Section 5.3.2). Therefore, a good code $\mathcal{C}$ for SSK-CM should be optimized taking into consideration both $d\left(\mathbf{X}_{1}, \mathbf{X}_{2}\right)$, and the diversity order achievable by $A^{\prime}$. To improve performance, codes may be designed to exploit this diversity property.

\subsubsection{UWB Implementation and Analysis}

In the thesis, we presented the simple Rayleigh fading model, to obtain strong theoretical background for SSK modulation. However, due to UWB advantages (fixed number of RF chains), and simplicity of SSK integration in UWB framework, an SSK presentation under UWB conditions is also an important contribution. 


\section{Bibliography}

[1] Apple Inc., ht.tp://www.apple.com/ (current July 09, 2008).

[2] P. Wolniansky, G. Foschini, G. Golden, and R. Valenzuela, "V-BLAST: An architecture for realizing very high data rates over the rich-scattering wireless channel," in Proc. International Symposium on Signals, Systems, and Electronics (ISSSE'98), Pisa, Italy, pp. 295-300, Sept.-Oct. 1998.

[3] S. M. Alamouti, "A simple transmit diversity technique for wireless communications," IEEE J. Sel. Areas Commun., vol. 16, no. 8, pp. 1451-1458, Oct. 1998.

[4] V. Tarokh, A. Naguib, N. Seshadri, and A. R. Calderbank, "Combined array processing and space-time coding," IEEE Trans. Inform. Theory, vol. 45, pp. 1121-1128, May 1999.

[5] DENSO Corp., http://www.globaldenso.com/en/ (current July 09, 2008).

[6] General Motors Corp., htt.p://www.gm.com/ (current July 09, 2008). 
[7] R. Böhnke, D. Wübben, V. Kühn, and K. D. Kammeyer, "Reduced complexity MMSE detection for BLAST architectures," in Proc. IEEE Globecom'03, San Francisco, California, USA, Dec. 2003.

[8] V. Tarokh, H. Jafarkhani, and A. R. Calderbank, "Space-time block code from orthogonal designs," IEEE Trans. Inform. Theory, vol. 45, pp. 1456-1467, July 1999.

[9] R. Mesleh, H. Haas, Y. Lee, and S. Yun, "Interchannel interference avoidance in mimo transmission by exploiting spatial information," Proc. of PIMRC'05, Sept. 11-14, 2005.

[10] Agilent Technologies, "E4438C-419 Signal Studio for 3GPP W-CDMA HSPA Online Documentation," Available: http://wireless.agilent.com/wireless/helpfiles/opt419/opt419.htm (current July $09,2008)$.

[11] A. F. Molisch and M. Z. Win, "MIMO systems with antenna selection," IEEE Microw. Mag., vol. 5, no. 1, pp. 46-56, Mar. 2004.

[12] R. Heath and A. Paulraj, "Antenna selection for spatial multiplexing systems based on minimum error rate," in Proc. IEEE Int. Contr. Conf., vol. 7, Helsinki, Finland, June 2001, pp. 2276-2280. 
[13] R. W. Heath, Jr., S. Sandhı, and A. Paulraj, "Antenna selection for spatial multiplexing with linear receivers," IEEE Commun. Letters, vol. 5, no. 4, pp. 142-144, Apr. 2001.

[14] A. Ghrayeb and T.M. Duman, "Performance analysis of MIMO systems with antenna selection over quasi-static fading channels," IEEE Trans. Vehicular Technology, vol. 52, pp. 281-288, Mar. 2003.

[15] X. N. Zeng and A. Ghrayeb, "Performance bounds for space-time block codes with receive antenna selection," IEEE Trans. Inf. Theory, vol. 50, no. 9, pp. 2130-2137, Sep. 2004.

[16] R. W. Heath, Jr and D. J. Love, "Multimode antenna selection for spatial multiplexing systems with linear receivers," IEEE Trans. Signal Process., vol. 53, no. 8, pp. 3042-3056, Aug. 2005.

[17] H. Shen and A. Ghrayeb, "Analysis of the outage probability for MIMO systems with receive antenna selection," IEEE Trans. Veh. Technol., vol. 55, pp. 14351441, July 2006.

[18] A. Sanei, A. Ghrayeb, Y. Shayan, and T. M. Duman, "On the diversity order of space-time trellis codes with receive antenna selection over fast fading channels," IEEE Trans. Wireless Commun., vol. 5, no. 7, pp. 1579-1585, July 2006.

[19] Y. A. Chau, and S.-H. Yı, "Space modulation on wireless fading channels," IEEE 54th VTC' 01 (Fall), vol.3, pp.1668-1671, 2001. 
[20] Y. A. Chau, and S.-H. Yu, "Space shift keying modulation" US Patent Application Publication, \# 0094783, Jul. 2002.

[21] H. Haas, E. Costa, and E. Schulz, "Increasing spectral efficiency by data multiplexing using antenna arrays," Proc.of PIMRC'02, vol. 2, pp. 610-613, Sept. $15-18,2002$.

[22] R. Mesleh, H. Haas, C. Ahn, and S. Yun, "Spatial modulation - a new low complexity spectral efficiency enhancing technique," First international Conference on Communications and Networking in China (ChinaCom'06), pp. 1-5, Oct. 2006.

[23] R. Mesleh, H. Haas, S. Sinanović, C. W. Ahn and S. Yun, "Spatial modulation," IEEE Trans. Vehicular Technology, vol. 57, no. 4, pp. 2228 - 2241, July 2008.

[24] R. Mesleh, H. Haas, C. W. Ahn, and S. Yun, "Spatial modılation-OFDM," In the Proceedings of the 11th International OFDM-Workshop 2006 (InOWo'06), pp. 288-292, Aingust, 2006.

[25] S. Ganesan, R. Mesleh, H. Haas, C.W. Ahn, S. Yun, "On the performance of spatial modulation OFDM," Fortieth Asilomar Conference on Signals, Systems and Computers(ACSSC' '06), pp.1825-1829, Oct.-Nov. 2006.

[26] T. M. Duman and A. Ghrayeb, Coding for MIMO Communication Systems. Wiley 2007. 
[27] J. Jeganathan, A. Ghrayeb, and L. Szczecinski, "Spatial modulation: Optimal detection and performance analysis," IEEE Commun. Letters, (to appear) vol. 12, no. 8, August 2008.

[28] J.G. Proakis, Digital Communications, (4th ed.) McGraw-Hill, New York, 2001.

[29] M.-S. Alouini and A. Goldsmith, "A unified approach for calculating error rates of linearly modulated signals over generalized fading channels," IEEE Trans. Commun., vol. 47, no. 9, pp. 1324-1334, September 1999.

[30] J. Jeganathan, A. Ghrayeb, and L. Szczecinski, "Space shift keying modulation for MIMO channels, submitted to IEEE Trans. Wireless Commun., June 2008.

[31] J. Jeganathan, A. Ghrayeb, and L. Szczecinski, "Generalized space shift keying modulation for MIMO channels, IEEE International Symposium on Personal, Indoor and Mobile Radio Communications (PIMRC'08), Cannes, France, Sept. 2008.OFDM," Fortieth Asilomar Conference on Signals, Systems and Computers, pp. 1825-1829, Oct.-Nov. 2006.

[32] A. Goldsmith and S. Chua, "Variable-rate variable-power M-QAM for fading channels," IEEE Trans. Commun., vol. 45, pp. 1218-1230, Oct. 1997.

[33] D. J. Love, R. W. Heath, W. Santipach, and M. L. Honig, "What is the value of limited feedback for MIMO channels?," IEEE Commun. Mag., vol. 42, no. 10, pp. 54-59, Oct. 2004. 
[34] S. Kay, Fundamental of Statistical Signal Processing: Estimation Theory, vol. 1, Prentice Hall, 1993.

[35] M. Biguesh and A. B. Gershman, "MIMO channel estimation: Optimal training and tradeoffs between estimation techniques," in Proc. ICC, Paris, France, Jun. 2004, vol. 5, pp. 2658-2662.

[36] D. Shiu, G. Foschini, M. Gans and J. Kahn "Fading correlation and its effect on the capacity of multi-element antenna systems," IEEE Trans. Commun., vol. 48, no. 3, pp. 102-513, March 2000.

[37] S. L. Loyka, "Channel capacity of MIMO architecture using the exponential correlation Inatrix," IEEE Commun. Lett., vol. 5, pp. 369-371, Sept. 2001.

[38] A. Forenza, D. J. Love, and R. W. Heath, Jr., "A low complexity algorithm to simulate the spatial covariance matrix for clustered MIMO channel models," in Proc. IEEE Veh. Technol. Conf., May 2004, vol. 2, pp. 889-893.

[39] K. I. Pedersen, P. E. Mogensen, and B. H. Fleury, "A stochastic model of the temporal and azimuthal dispersion seen at the base station in outdoor propagation environments," IEEE Trans. Vehicular Technology, vol. 49, pp. 437-447, Mar. 2000.

[40] G. Ungerboeck, "Channel coding with multilevel/phase signals", IEEE Trans. Inform. Theory, Vol. 28, no.1, pp. 55-67, Jan 1982. 
[41] S. Le Goff, A. Glavieıx, and C. Berrou, "Turbo-codes and high spectral efficiency modulation," in Proc. IEEE Int. Conf. Commun., 1994, pp. 645-649.

[42] P. Robertson and T. Woerz, "Novel bandwidth efficient coding scheme employing turbo codes," in Proc. IEEE Int. Conf. Commun., 1996, pp. 962-967.

[43] S. Benedetto, D. Divsalar, G. Montorsi, and F. Pollara, "Parallel concatenated trellis-coded modılation," in Proc. IEEE Int. Conf. Commun., 1996, pp. 974-978.

[44] T. M. Duman, "Turbo codes and turbo coded modulation systems: Analysis and performance bounds," Ph.D. dissertation, Northeastern University, Dept. of Electrical and Computer Engineering, Boston, MA, 1998.

[45] A. Stefanov, and T.M. Duman, "Turbo-coded modulation for systems with transmit and receive antenna diversity over quasi-static fading channels: system model, decoding approaches, and practical considerations," IEEE J. Sel. Areas in Commun., vol.19, no.5, pp.958-968, May 2001.

[46] E. Zehavi, "8-PSK Trellis Codes for a Rayleigh Channel," IEEE Trans. Comm., vol. 40, pp. 873-884, May 1992.

[47] G. Caire, G. Taricco, and E. Biglieri, "Bit-interleaved coded modulation," IEEE Trans. Inform. Theory, vol. 44, pp. 927-947, May 1998.

[48] S. Y. Le Goff, "Signal constellations for bit-interleaved coded modulation," IEEE Trans. Inform. Theory, vol. 49, pp. 307-313, Jan. 2003. 
[49] N. H. Tran and H. H. Nguyen, "Design and performance of BICM-ID systems with hypercube constellations," IEEE Trans. Wireless Commun., vol. 5, no. 5, pp. 1169-1179, May 2006.

[50] F. Schreckenbach and G. Bauch, "Bit-interleaved coded irregular modulation," Euro. Trans. Telecomms., vol. 17, no. 2, pp. 269-282, Mar.-Apr. 2006.

[51] X. Li and J. A. Ritcey, "Bit-interleaved coded modulation with iterative decoding," in Proc. IEEE Int. Conf. on Commun. (ICC'99), June 1999, pp. 858-862.

[52] T. M. Duman and M. Salehi, "Performance bounds for turbo-coded modulation systems," IEEE Trans. Commun., vol. 47, pp. 511-521, Apr. 1999.

[53] Lin S., and Costello D. J. Jr., Error Control Coding. (2nd ed.) Pearson Prentice Hall, New Jersey: Upper Saddle River, 2004.

[54] L. Szczecinski, R. Bettancourt, and R. Feick, "Probability density function of reliability metrics in BICM with arbitrary modulation: closed-form through algorithmic approach," IEEE Trans. Commun. (in press), May 2008.

[55] A. Martinez, A. Guillén i Fàbregas, and G. Caire, "Error probability of bitinterleaved coded modulation," IEEE Trans. Inform. Theory, vol. 52, no.1, pp. 262-271, Jan. 2006.

[56] A. Chindapol and J. A. Ritcey, "Design, analysis and performance evaluation for BICM-ID with square QAM constellations in Rayleigh fading channels," IEEE J. Select. Areas Commun., vol. 19, pp. 944-957, May 2001. 
[57] A. Martinez, A. Guillén i Fàbregas, and G. Caire, "A closed-form approximation for the error probability of BPSK fading channels," IEEE Trans. Wireless Commun., vol. 6, no.6, pp. 2051-2054, Jun. 2007. 